\title{
Final Report on the Electrochemical Treatment of Alkaline Nuclear Waste
}

by

D. T. Hobbs

Westinghouse Savannah River Company

Savannah River Site

Aiken, South Carolina 29808

This paper was prepared in connection with work done under the above contract number with the U.S. Department of Energy. By acceptance of this paper, the publisher and/or recipient acknowledges the U. S. Government's right to retain a nonexclusive, royalty-free license in and to any copyright covering this paper, along with the right to reproduce and to authorize others to reproduce all or part of the copyrighted paper. 


\section{DISCLAIMER}

This report was prepared as an account of work sponsored by an agency of the United States Government. Neither the United States Government nor any agency thereof, nor any of their employees, makes any warranty, express or implied, or assumes any legal liability or responsibility for the accuracy, completeness, or usefulness of any information, apparatus, product, or process disclosed, or represents that its use would not infringe privately owned rights. Reference herein to any specific commercial product, process, or service by trade name, trademark, manufacturer, or otherwise does not necessarily constitute or imply its endorsement, recommendation, or favoring by the United States Government or any agency thereof. The views and opinions of authors expressed herein do not necessarily state or reflect those of the United States Government or any agency thereof.

This report has been reproduced directly from the best available copy.

Available to DOE and DOE contractors from the Office of Scientific and Technical Information, P.O. Box 62, Oak Ridge, TN 37831; prices available from (615) 576-8401.

Available to the public from the National Technical Information Service, U.S. Department of Commerce, 5285 Port Royal Road, Springfield, VA 22161. 


\section{DISCLAIMER}

Portions of this document may be illegible electronic image products. Images are produced from the best available original document. 
Distribution: $\quad$ K. Gerdes, DOE-HQ

.W. L. Kuhn, PNNL

W. E. Lawrence, PNNL

R. E. White, USC

S. V. Johnson, DOE-SR, 703-A

E. A. Korzun, 703-H

L. M. Papouchado, 773-A

W. L. Tamosaitis, 773-A

S. D. Fink, 773-A

C. B. Hatfield, 773-41A

D. T. Hobbs, 773-A

A. Patterson (IWT-LWP Files), 773-A TIM, 773-52A 


\section{WSRC-TR-96-0366 Th}

Keywords: Electrolysis, Alkaline, Salt Solution, Nitrate, Nitrite, Organics, Modeling, Metals Waste Management

Retention time: permanent

October 30, 1996

TO: $\quad$ S. D. Fink, $773-A$<smiles>CCC1CCC12CCC2</smiles>

FROM: D. T. Hobbs, 773-A

\section{Final Report on the Electrochemical Treatment of Alkaline Nuclear Wastes (U)}

Attached is the final report prepared by the University of South Carolina on work completed during SCUREF contract \#AA00900T, Task Order \#112, investigating the electrochemical destruction of organic, nitrate and nitrite compounds, the removal of metals by electrodeposition and the development of engineering models of electrolytic processes.

Authorized Derivative Classifier

Authorized Reviewing Official

Savannah River Technology Center

Westinghouse Savannah River Company

Aiken, SC 29802

Prepared for the Department of Energy Office of Technology Development, Office of Environmental Management under Contract DE-AC09-88SR18035 
Electrochemical Treatment of Alkaline Nuclear Wastes

\section{FINAL REPORT}

SCUREF Contract \#AA009-00T

Task Order \#112

October, 1996

by

J. W. Van Zee

Department of Chemical Engineering

University of South Carolina

Columbia, SC 29208

Submitted to D. T. Hobbs

Westinghouse Savannah River Company 


\section{Summary}

This report describes the work on the electrochemical treatment of liquid alkaline $\mathrm{NaNO}_{3} / \mathrm{Na} \mathrm{NO}_{2}$ wastes. It is the sixth and final report for the SCUREF/USC/Westinghouse Savannah River Company contract AA009-00T, Task \#112. This contract began in May 1993 and ended in September 1996. Regular monthly reports have been submitted to document the tasks outlined in the contract. Previous reports documenting intermediate milestones have been submitted:

1. "A Parallel Plate Electrochemical Reactor Model for the Destruction of Nitrate Wastes in Alkaline Solutions," D.Coleman and R.E. White, Milestone Report submitted December 1993.

2. "A Flowsheet Model for the Electrochemical Treatment of Liquid Radioactive Wastes," S. Prasad, J. Weidner, A. Farell and R.E. White, Milestone Report submitted September 1994.

3. "Evaluation of Porous Cathodes Used for the Electrochemical Reduction of Nitrate and Nitrite in Liquid Wastes," K. Jha and J. Weidner, Interim and Milestone Reports, September 1994 and December 1995.

4. "A Dynamic Flowsheet Model for the Electrochemical Treatment of Liquid Radioactive Wastes," S. Prasad, J. Weidner, A. Farell and R.E. White, Milestone Report, June 1995.

5. "Mathematical Model for the Destruction of Tetrasodium Ethylenediaminetetraacetate in a Parallel Plate Electrochemical Reactor," S.N. Pakalapati, B.N. Popov and R.E. White, Milestone Report, August 1995.

This report presents a comprehensive consistent set of data from a bench scale electrochemical cell. The cell and the reservoirs constitute the batch reactor system that was used for the electrochemical reduction of $\mathrm{NO}_{3}^{-}$and $\mathrm{NO}_{2}^{-}$to $\mathrm{N}_{2}, \mathrm{~N}_{2} \mathrm{O}$, and $\mathrm{NH}_{3}$. These data are used to estimate the electrochemical kinetic parameters for the five cathodic reactions that occur at a nickel electrode. A model is presented and used with a non linear least squares objective function and these data to obtain these parameters.

The model predictions with these parameters are shown to agree well with separated batch reactor data at a constant current of $0.25 \mathrm{~A} / \mathrm{cm}^{2}$. The model also predicts the changes in the liquid and gas phase products when the current density is decreased from $0.25 \mathrm{~A} / \mathrm{cm}^{2}$ to 0.05 $\mathrm{A} / \mathrm{cm}^{2}$. It is shown that this decrease in the current density, at the appropriate time predicted by the model, will decrease the production of $\mathrm{H}_{2}$ and thereby increase the efficiency of the nitrate/nitrite destruction process. The model and the estimated parameters can be used to optimize the design and operation of a waste treatment facility. 


\section{Table of Contents}

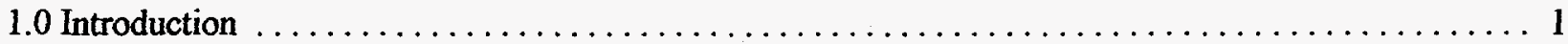

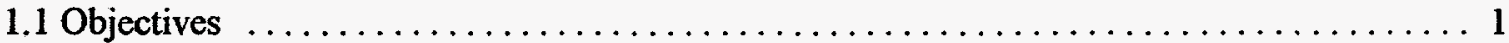

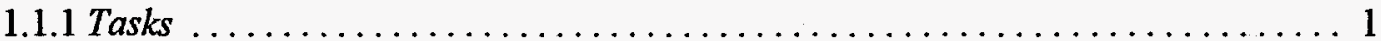

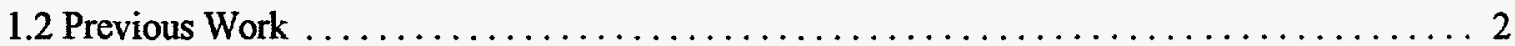

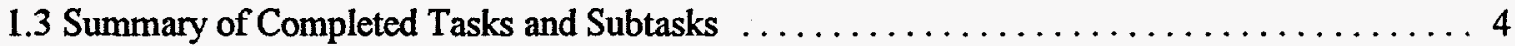

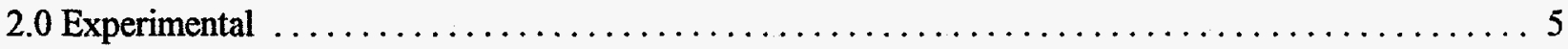

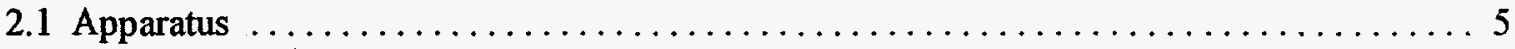

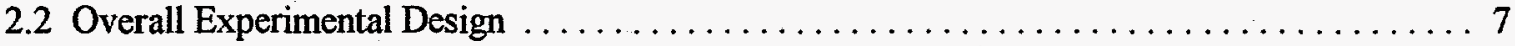

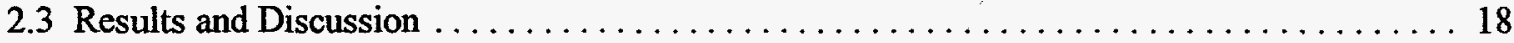

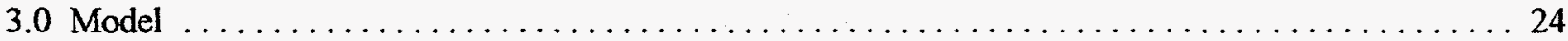

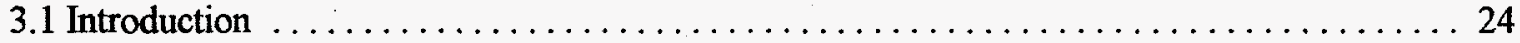

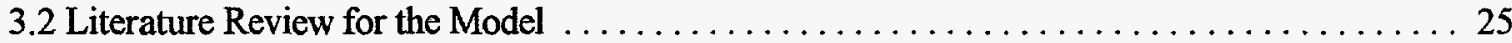

3.2.1 Boundary Layer and Algebraic Models ..................... 25

3.2 .2 Batch Reactor Models ................................ 25

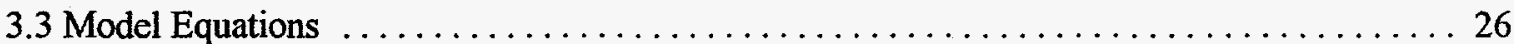

3.3.1 Model Assumptions ................................ 27

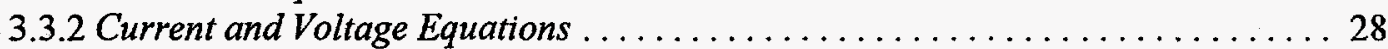

3.3.3 Reservoir Mass and Energy Balances ....................... 31

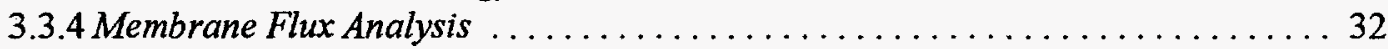

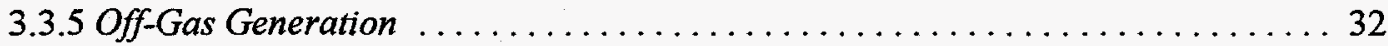

3.3.6 Solution Technique and Model Algorithm ................. 34

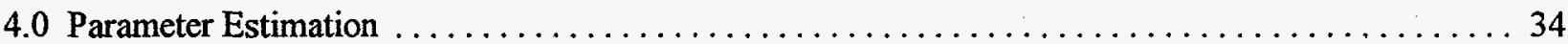

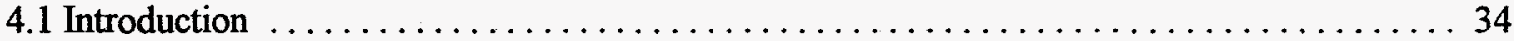

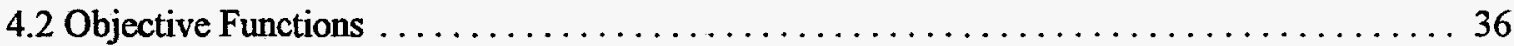

4.3 Results from Model Regression of Experimental $\mathrm{NO}_{2}^{-}$Data $\ldots \ldots \ldots \ldots \ldots \ldots \ldots \ldots 40$

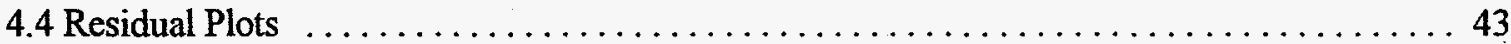

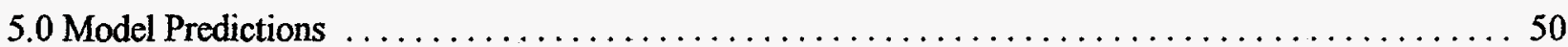

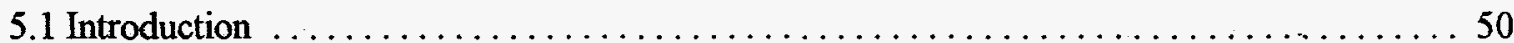

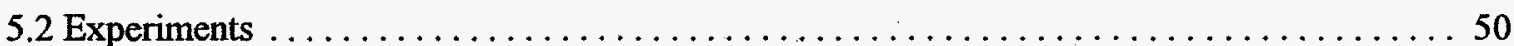

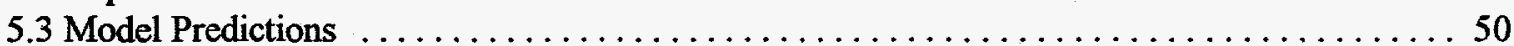

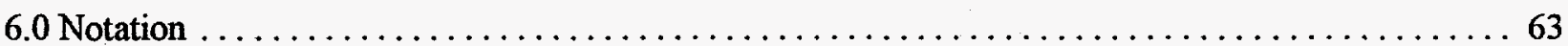

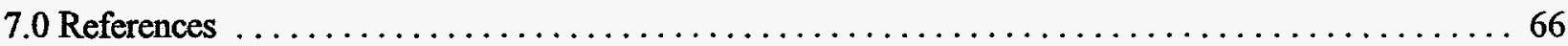

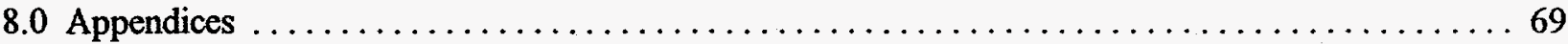

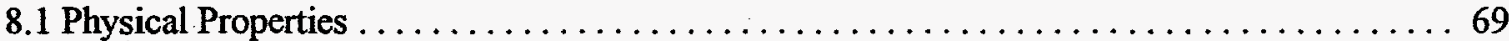

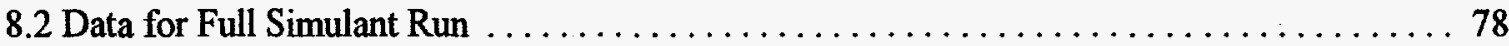

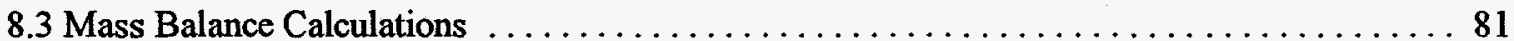

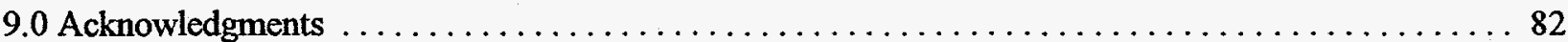




\subsection{Introduction}

The electrochemical treatment of liquid radioactive wastes has become a topic of scientific and environmental interest in recent years. In particular, methods of electrochemically reducing alkaline $\mathrm{NaNO}_{3} / \mathrm{NaNO}_{2}$ wastes to nitrogen products and $\mathrm{NaOH}$ hold promise for decreasing the waste volume and for possible reuse, after evaporation, as a pure stream of concentrated $\mathrm{NaOH}$ [1-5]. Experimental data collected from a divided parallel plate electrolyzer, operated in a batch mode, indicated that the nitrate and nitrite reactions were controlled by both kinetics and mass transfer [2]. Although these results suggest that the process is feasible for treatment of wastes, design of a scale-up facility is complicated by the absence of kinetic data. In this report, experimental data is presented that can be used to estimate these kinetic parameters by using a mathematical model to analyze new data from batch experiments on an MP cell.

The set of electrochemical reactions, consistent with the observation of $\mathrm{NH}_{3}, \mathrm{~N}_{2} \mathrm{O}, \mathrm{N}_{2}$ and $\mathrm{H}_{2}$ by Genders et al. [3] could be written:

$$
\begin{array}{lr}
1 / 2 \mathrm{NO}_{3}^{-}+1 / 2 \mathrm{H}_{2} \mathrm{O}+\mathrm{e}^{-}=1 / 2 \mathrm{NO}_{2}^{-}+\mathrm{OH}^{-} & 0.011 \\
1 / 6 \mathrm{NO}_{2}^{-}+5 / 6 \mathrm{H}_{2} \mathrm{O}+\mathrm{e}^{-}=1 / 6 \mathrm{NH}_{3}+7 / 6 \mathrm{OH}^{-} & -0.165 \\
1 / 3 \mathrm{NO}_{2}^{-}+2 / 3 \mathrm{H}_{2} \mathrm{O}+\mathrm{e}^{-}=1 / 6 \mathrm{~N}_{2}+4 / 3 \mathrm{OH}^{-} & 0.406 \\
1 / 2 \mathrm{NO}_{2}^{-}+3 / 4 \mathrm{H}_{2} \mathrm{O}+\mathrm{e}^{-}=1 / 4 \mathrm{~N}_{2} \mathrm{O}+3 / 2 \mathrm{OH}^{-} & 0.151 \\
\mathrm{H}_{2} \mathrm{O}+\mathrm{e}^{-}=1 / 2 \mathrm{H}_{2}+\mathrm{OH}^{-} & -0.828
\end{array}
$$

The oxidation of $\mathrm{OH}^{-}$was observed at the anode:

$$
\mathrm{OH}^{-} * 1 / 4 \mathrm{O}_{2}+1 / 2 \mathrm{H}_{2} \mathrm{O}+\mathrm{e}^{-}
$$

where $\mathrm{U}^{0}$ is the standard reduction potential for each reaction at $25^{\circ} \mathrm{C}$.

\subsection{Objectives}

The objectives of this project were as follows:

(1) To demonstrate that electrochemical processes can be used at the laboratory scale for the destruction of nitrate, nitrite, and organic compounds from simulated waste streams that are produced at the Savannah River Site and throughout the DOE complex.

(2) To develop engineering models and flowsheets which can be used for optimization, design, and cost estimation of pilot plant and full-scale equipment for an electrochemical treatment process.

(3) To evaluate other unit operations such as off-gas treatment and recovery of chemicals with value (e.g., a strong caustic solution) which would be associated with the electrochemical processes.

\subsubsection{Tasks}

The following tasks were conducted:

(1) The optimum electrode materials, electrocatalysts, reactor designs, and operating conditions 
for the destruction of nitrate and nitrite were determined. To do this, the following types of electrodes were studied: planar, flow-through porous, and a gas-diffusion electrode serving as the anode. The electrochemical technology for the destruction of organic compounds such as ethylenediaminetetraacetic acid (EDTA) from alkaline waste solutions was identified.

(2) Engineering models for the electrochemical reactors and dynamic process flowsheets for the electrochemical treatment of liquid waste were developed. The cell designs that were considered in the engineering models development include a parallel-plate cell and flow-through porous cathodes cells. Flowsheets were developed that included engineering models for each unit operation so that process optimization, design, and cost estimation studies can be carried out. Upon identification/selection of unit operation technology, addition data needed for the models was determined and experimental work was conducted to provide the necessary data.

(3) The materials of construction of the cells, the electrodes, associated piping and other unit operations were selected to attempt to prevent degradation of these components during service.

(4) Other unit operations which are associated with the electrochemical processes such as off-gas treatment and the recovery of chemicals with value were identified.

(5) The electrochemical technology for the destruction of organic compounds in alkaline-waste streams was evaluated.

(6) The electrochemical reaction rates for the destruction of selected organic compounds in Hanford wastes were determined.

(7) Organic oxidation reactions were included in the engineering and dynamic flowsheet models for the electrochemical treatment processes.

(8) Assistance was provided for the design of pilot-scale and full-scale electrochemical treatment facilities.

\subsection{Previous Work}

Experimental work has been performed on the electrochemical reduction of nitrate in acidic media as a means of producing ammonia and nitrous oxide [6-8]. Cox and Brajter [6] performed cyclic voltammetry, phase selective ac polarography, and conductance measurements to confirm the catalytic effect of $\mathrm{Zr}$ (IV) in the reduction of nitrate to ammonia at mercury electrodes. Pletcher and Poorabedi [7] performed cyclic voltammetry and rotating disc experiments in a two-compartment cell to demonstrate the reduction of nitrate to ammonia at a copper cathode in aqueous acidic perchlorate and sulphate media. Xing et al. [8] used rotating ring-disk techniques to determine the electrocatalytic effects of cadmium for the reduction of nitrate at gold and silver surfaces in acidic solutions.

With increasing interest in the electrochemical treatment of low-level radioactive waste, studies have been conducted on the electrochemical reduction of nitrates and nitrogen 
containing compounds in alkaline media. Johnson and Sawyer [9] presented cyclic voltammetry work on the electrochemical reduction of $\mathrm{N}_{2} \mathrm{O}$ at a platinum electrode to $\mathrm{OH}^{-}$and $\mathrm{N}_{2}$. Recent work has focussed on the cathodic materials used in the reduction of nitrates and nitrites [3,10-14]. Horanyi and Rizmayer [10] presented polarization curves for platinized platinum cathodes, in low concentrations alkaline solutions containing $\mathrm{NO}_{3}^{-} / \mathrm{NO}_{2}^{-}$. They concluded that the maxima in the polarization curves were due to adsorption competition between the reacting species and the supporting electrolyte. As discussed below, and in Prasad et al. [5], these maxima can also be a result of competing effects of diffusion and migration of $\mathrm{NO}_{3}{ }^{-}$from a cathode at which simultaneous reactions occur.

Hobbs and Ebra [2] proposed a process flowsheet for the electrochemical destruction of nitrates and nitrites in low-level radioactive waste. They concluded that this process could produce a significant reduction in the volume of the waste incorporated into Saltstone for permanent disposal. Li et al. [11] used cyclic voltammetry and bulk electrolysis at low concentrations (i.e., $0.015 \mathrm{M} \mathrm{NaNO}_{2}$ and $0.01 \mathrm{M} \mathrm{NaNO}_{3}$ ) to study the reduction of nitrate and nitrite on a platinized nickel cathode. This electrode showed high current efficiencies for the production of nitrogen gas and minimal ammonia production at a temperature of $80^{\circ} \mathrm{C}$ and at a current density of $0.107 \mathrm{~A} / \mathrm{cm}^{2}$ in $3.0 \mathrm{M} \mathrm{NaOH}$. At higher current densities $\left(0.466 \mathrm{~A} / \mathrm{cm}^{2}\right)$ ammonia was observed as the major electrolysis product.

Li et al. [12] examined the efficiency of nickel, lead, zinc, and iron cathodes in the reduction of nitrate ions. This work suggested using a zinc or lead cathode and a nickel anode would improve the efficiency of the process. Later Li et al. [13] discussed the use of cobaltcyclam as an electrocatalyst for the reduction of nitrate and nitrite to form hydroxylamine and ammonia for which a reaction mechanism was proposed.

Cattarin [14] presented work that showed that with the proper choice of cathode material and applied potential various products may be obtained with good selectivity. For example, nitrate may be reduced to nitrite using a silver cathode, to ammonia using a copper or zinc cathode, and to nitrogen using a nickel cathode.

Previous work [10-14], in addition to the work performed by Genders et al. [3], has examined cathode/anode combinations to determine those most favorable for the reduction of $\mathrm{NO}_{3}{ }^{-} / \mathrm{NO}_{2}{ }^{-}$and the minimization of the reduction of water. Cathode materials tested consisted of graphite [3], lead [3,12], nickel $[3,11,12]$, zinc [12,14], iron [12], copper [3,14], silver [14], gold [13], stainless steel [3], platinum [3,11] and platinized nickel [11], and the lead cathode was found to be a feasible material [3] but environmental considerations may limit its usefulness. While these papers give an indication of the rate of nitrate and nitrite destruction, these experiments do not permit the determination of exchange current densities and transfer coefficients because reference electrodes were not used and polarization data are not presented.

In this project, the anode materials that were tested consisted of nickel, stainless steel, platinum and platinized titanium. Also, a gas diffusion hydrogen anode was tested as documented in the monthly reports. In this report, only a nickel cathode and a stainless steel anode are considered. However, the methods discussed in the sections that follow to determine the kinetic parameters of the electrochemical reactions may be applied to any electrode materials. 


\subsection{Summary of Completed Tasks and Subtasks}

Monthly reports documenting the accomplishments and progress on the project have been submitted throughout the period of performance. Publications, presentations, milestone reports, and student theses and dissertations have been described in those reports. The tasks that have been completed during the period of performance are listed below.

Task Activity

1. Laboratory-scale Testing

a. Exploratory evaluation of packed-bed and fluidized-bed electrodes (subcontracted work to Texas A\&M University)

b. Exploratory evaluation of flow-through porous metal electrodes

c. Evaluate electrocatalysts

d. Exploratory evaluation of gas diffusion anodes

e. Build laboratory scale process to evaluate various cell designs, electrodes, and electrocatalysts (e.g., a cell with flow through porous electrodes)

f. Initiate studies to determine the feasibility of using an electrochemical cell to destroy sodium teraphenyl borate and phenol in a caustic solution

g. EDTA destruction experiments

h. Determine reaction rates for destruction of organic compounds in Hanford Wastes

i. Thorough evaluation of flow-through porous metal electrodes

j. Thorough evaluation gas diffusion anodes

$k$. Build second laboratory scale process to evaluate various organic cell designs, electrodes, and operating conditions
Date Completed
$3 / 31 / 95$

$9 / 30 / 94$

$9 / 30 / 94$

$9 / 30 / 94$

$3 / 31 / 95$

$3 / 31 / 95$

$9 / 30 / 95$

$9 / 30 / 96$

$9 / 30 / 95$

$9 / 30 / 95$

$4 / 30 / 95$

$12 / 31 / 93$

$9 / 30 / 95$

$12 / 31 / 93$

$8 / 31 / 94$

$9 / 30 / 93$

$9 / 30 / 95$

$5 / 31 / 95$

9/30/95

$9 / 30 / 95$

$9 / 30 / 95$

3. Evaluation of Materials of Construction

a. Use standard practice to select electrodes that will be suitable for use in strong caustic solutions with additives of interest in this project.

b. Use standard practice to select cell components

$9 / 30 / 96$

$9 / 30 / 95$

c. Use standard practice to select connecting piping systems and tank

$9 / 30 / 95$ 


\section{2-1. Apparatus}

\subsection{Experimental}

Figure 1 shows a schematic diagram of the apparatus used to collect experimental data under batch operation. Storage tanks \#1 and \#2 correspond to the airtight anolyte and catholyte reservoirs, respectively. For batch operation, the solutions were returned to tanks \#1 and $\# 2$ through valves during the run. The catholyte was pumped through valves 10 and 13 into the reactor and then returned to reservoir $\# 2$ through valve 16 . Similarly, the anolyte solution was pumped through valves 9 and 12 , into the anolyte chamber and then returned to reservoir \#1 through valve 14. Valves 11 and 15 remained closed during divided cell operation. Valves 7 and 8 were adjusted to insure that the reservoirs remained well mixed and they allow the use of centrifugal pumps (March Mfg. Inc. Model TE-50-MD).

Thermocouples were installed in the storage tanks and in the exit of the electrolytic cell for the addition of temperature control using stainless steel heat exchangers immersed in constant temperature baths not shown in the figure.

Liquid sample ports (valves 2 and 5) were fitted on each storage tank. To start an experiment, tanks \#1 and \#2 were filled with anolyte and catholyte solutions with valves 1 and 4 closed. The centrifugal pumps were turned on and the rotameters and valves 12 and 13 were adjusted to give the desired flow rates (about $6.3 \times 10^{-5} \mathrm{~m}^{3} / \mathrm{s}$ ). The current was applied to the electrochemical cell with either an EG\&G Princeton Applied Research Potentiostat/ Galvanostat (Model 273A) for current densities less than $0.1 \mathrm{~A} / \mathrm{cm}^{2}$ or a Lambda power supply (Model LK 362-FM) for current densities between 0.1 and $0.25 \mathrm{~A} / \mathrm{cm}^{2}$.The current was measured with a Ram Meter $50 \mathrm{Amp} / 50 \mathrm{mV} \pm 0.1 \%$ shunt. Rotameters $\mathrm{A}$ and $\mathrm{B}$ were calibrated with the anolyte and catholyte solutions manually at room temperature.

The off-gases flowed through the airtight storage tanks, into water chilled condensers and into the atmosphere through bubble meters. Septums were fitted into this flow stream to allow for gas chromatograph analysis. Ports not shown in the figure were installed above the liquid electrolyte levels in the storage tanks to allow the gas injection of a metered flow of argon into the off-gas stream. Calibrated mass flow meters (Omega Model FMA-762V) were used to fix the argon flowrate. The gas chromatograph was calibrated using standardized mixtures of argon and the off-gas species. Therefore, the composition of the off-gas and the molar flow rates of the individual off-gas species could be determined directly from the GC analysis. The bubble meters served as a check of the instantaneous off-gas flow rate.

In a typical experiment, the current density was held constant and anolyte and catholyte samples were collected every hour to obtain concentration data for the aqueous species. The dependent variables were the cell temperature, the transient liquid phase $\mathrm{NO}_{3}^{-}, \mathrm{NO}_{2}{ }^{-}, \mathrm{OH}^{-}$and $\mathrm{NH}_{3}$ concentrations, the off-gas mole fractions, the total gas flow rate, the changes in the anolyte and catholyte volumes, the total cell potential, and three solution potentials. These potentials were located adjacent to the cathode, adjacent to the catholyte side of the separator and adjacent to the anolyte side of the separator. Reservoir temperatures and cell voltages were recorded every hour during the experiment.

The concentrations of the nitrogen containing cathodic species (i.e., $\mathrm{NO}_{3}^{-}$and $\mathrm{NO}_{2}^{-}$) 


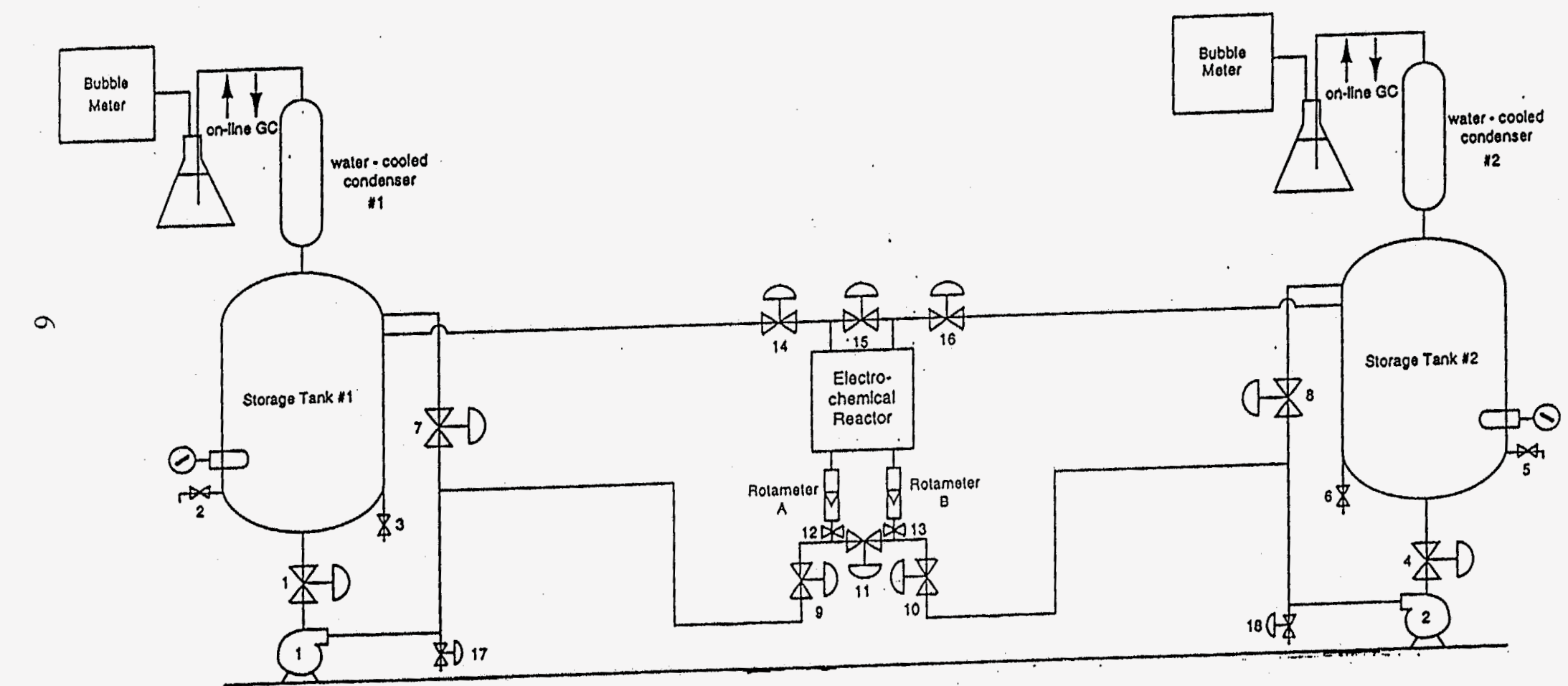

Figure 1. Schematic of the experimental apparatus 
were determined by ion chromatography and the concentration of $\mathrm{OH}^{-}$was determined by titration. Gas flow rates and mole fractions were measured using a gas chromatograph as discussed above. A Kjeldahl method [7] was used to measure the concentration of $\mathrm{NH}_{3}$ and $\mathrm{NH}_{4}{ }^{+}$in the catholyte. The volume change of the catholyte was determined by an ion chromatograph measurement of $\mathrm{Cl}^{-}$in the catholyte. That is, 16.5 grams of $\mathrm{NaCl}$ per liter of electrolyte were added at the beginning of the experiment to serve as a tracer for the catholyte volume. A more complete outline of the analytical operating procedures is given in reference [15].

The experimental data was obtained by galvanostatic operation of an Electrocell $\mathrm{AB}$ MP cell shown schematically in Figure 2. In the experiments with the MP cell, the anolyte and catholyte flow was upward through turbulence promoters (not shown in the Figure 2). The projected electrode area is $100 \mathrm{~cm}^{2}$ for both electrodes with a total separation of $1.25 \mathrm{~cm}$ equal to the sum of $S_{a}, S_{s}$, and $S_{c}$. A cationic membrane, Nafion 350 , was used as the separator. The superficial velocity of both electrolytes was held constant at $10.5 \pm 1.1 \mathrm{~cm} / \mathrm{sec}$ through the $6 \mathrm{~cm}^{2} / \mathrm{s}$ cross sectional area and this corresponds to a flow rate of approximately $6.3 \times 10^{-5} \mathrm{~m}^{3} / \mathrm{s}$ in all of the experiments.

Luggin capillaries were inserted in the anolyte and catholyte compartments of the MP electrolyzer as shown in Figure 3. These capillaries were at a distance of $0.3 \mathrm{~cm}$ from the separator and the electrode in each compartment. The capillaries fed $50 \mathrm{ml}$ beakers filled with anolyte and catholyte and fitted with $\mathrm{Ag} / \mathrm{AgCl}$ reference electrodes. During the experiments, the total potential difference between the anode and the cathode and the potential differences between the anode and the anode reference, the anode reference and the cathode reference, and the cathode reference and the cathode were measured.

Figure 3 also illustrates some of the processes occurring in the MP electrolyzer in which there is bulk convection of the electrolytes flowing upward through the cell. In the boundary layers, diffusion and migration occur in which the ionic species are transported to and from the electrode surface. Beyond the boundary layers, the concentrations of the ionic species maybe assumed to be that of the bulk solution. The potential drops continuously throughout the cell from anode to cathode but the largest gradients are assumed to occur in the boundary layers of the anode and cathode and within the Nafion membrane. Voltage losses also occur in the bulk anolyte and catholyte due to the conductivity of the electrolyte solutions and the porosity and tortuosity of the turbulence promoters.

\subsection{Overall Experimental Design}

Four types of batch experiments were performed:

(1) Short time (3-4 hour) experiments in which the current density was scanned in a stepwise manner between $1.0 \times 10^{-4}$ to $0.25 \mathrm{~A} / \mathrm{cm}^{2}$, with approximately 4 liters of $3.0 \mathrm{M}$ $\mathrm{NaOH}$ in both the anolyte and catholyte. These data are useful for determining the kinetics toward the end of a full batch experiment when $\mathrm{NaOH}$ is the primary reactant in the catholyte and anolyte, and this results in the reduction of water to $\mathrm{H}_{2}$ is the primary reaction cathode. Also, they can be used to determine the kinetics for the oxidation of $\mathrm{OH}^{-}$at the anode.

(2) Short time (3-4 hour) experiments, at a constant current density, with approximately 4 
Figure 2. Schematic of the MP electrolyzer

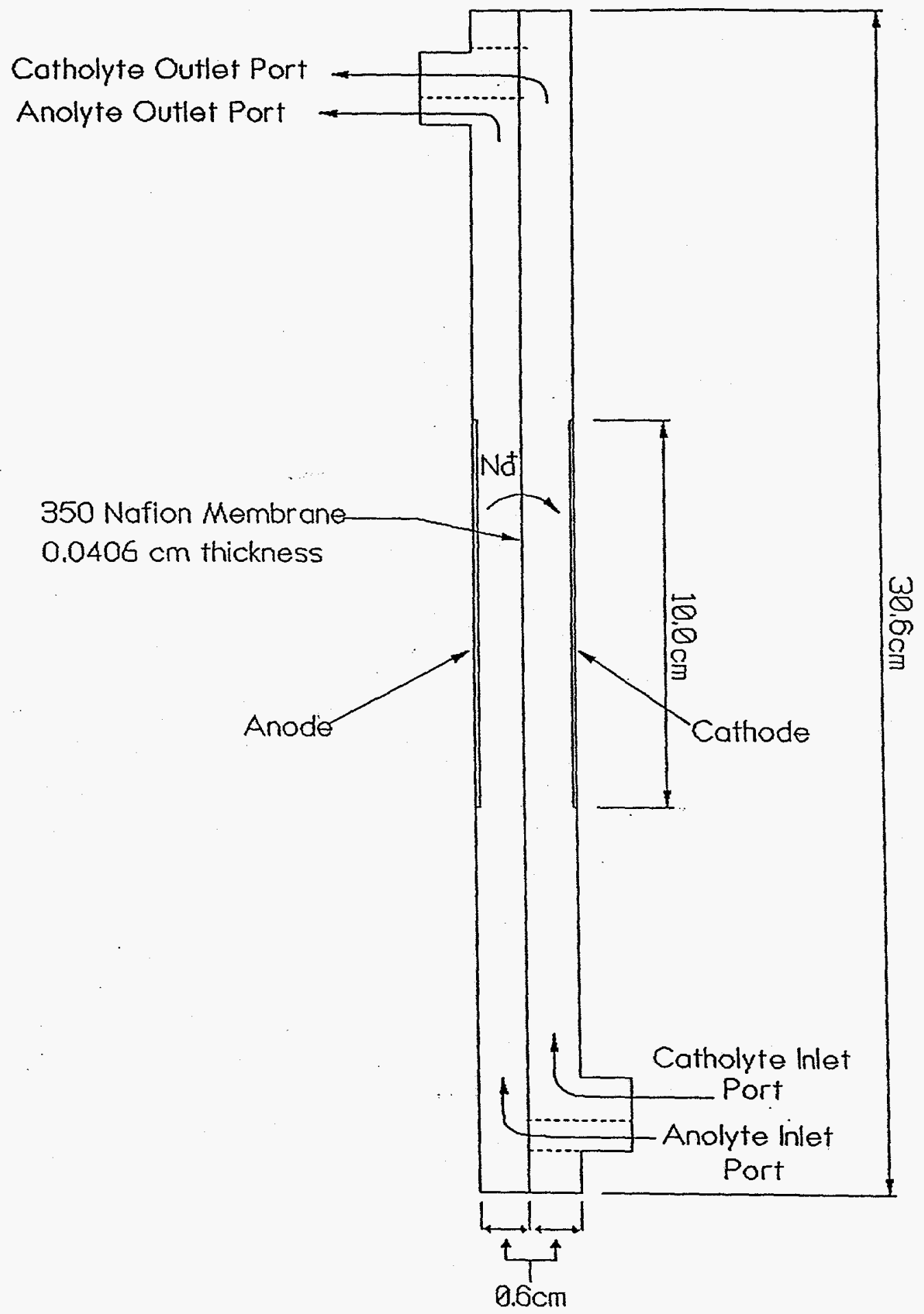


Anode
Reference

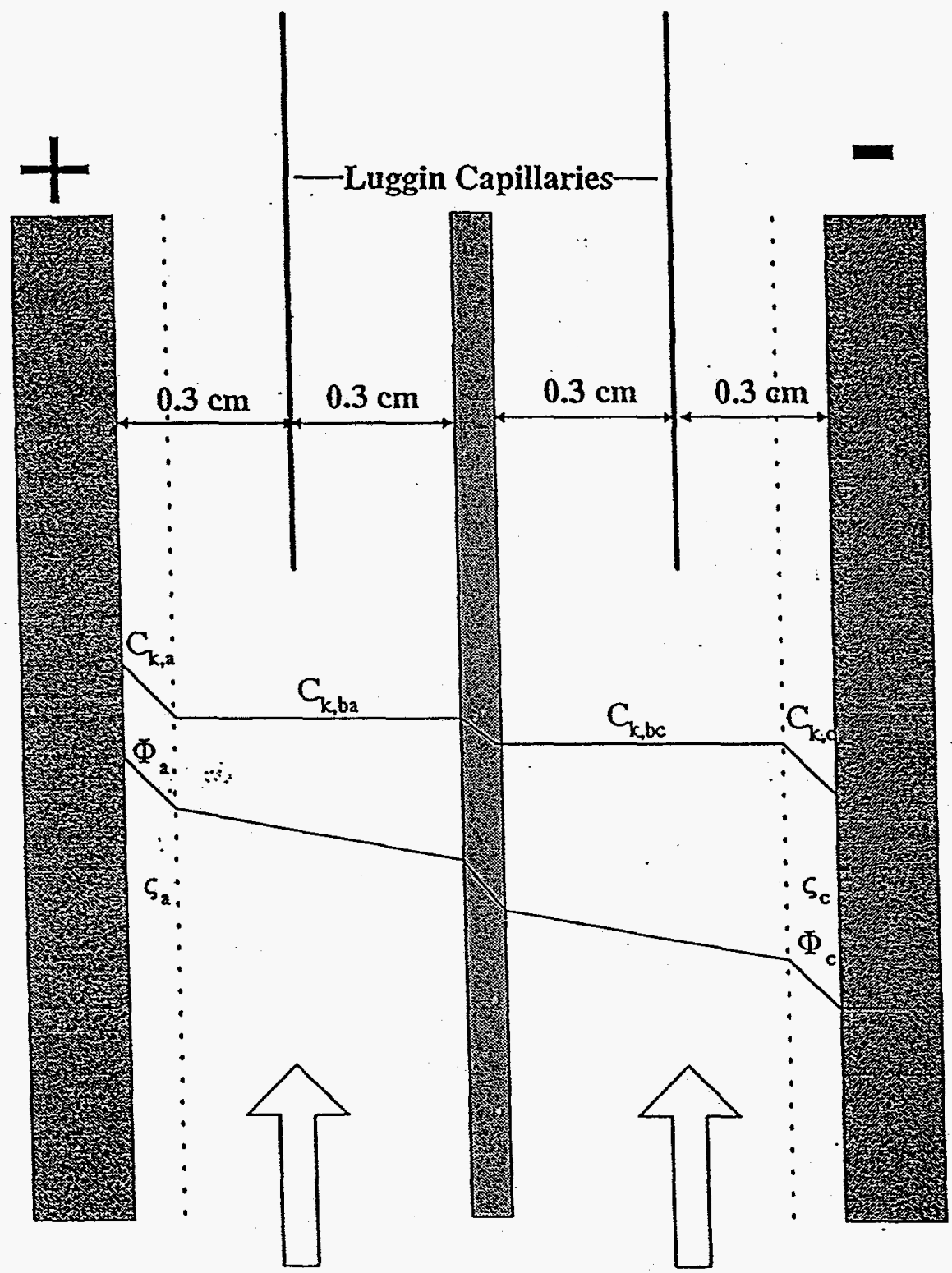

Anode
Cathode

Reference 
liters of $0.1 \mathrm{M}$ or $0.6 \mathrm{M} \mathrm{NaNO}_{2}$ in $3.0 \mathrm{M} \mathrm{NaOH}$ in the catholyte. Approximately, 2 liters of $3.0 \mathrm{M} \mathrm{NaOH}$ solution was used for the anolyte.

(3) Short time (3-4 hour) experiments, at a constant current density, with approximately 4 liters of $1.9 \mathrm{M}$ or $0.6 \mathrm{M} \mathrm{NaNO}_{3}$ in $3.0 \mathrm{M} \mathrm{NaOH}$ in the catholyte. Again, 2 liters of 3.0 $\mathrm{M} \mathrm{NaOH}$ solution was used for the anolyte.

(4) Full batch experiments (approximately 20 hours), at a constant current density, with starting concentrations of $1.9 \mathrm{M} \mathrm{NaNO}_{3}, 0.6 \mathrm{M} \mathrm{NaNO}_{2}$, and $1.3 \mathrm{NaOH}$ in the catholyte and 5.0 $\mathrm{M} \mathrm{NaOH}$ in the anolyte were used.

Replicates of these experiments were performed for all experiments. In experiments of type 2-4, current densities of 0.15 and $0.25 \mathrm{~A} / \mathrm{cm}^{2}$ were used. Experiments of type 3 were not conducted at low $\mathrm{NaNO}_{3}$ concentrations (i.e., less than $0.6 \mathrm{M}$ ) because water reduction is the major electrochemical reaction.

Experiments of type 1 can be used to estimate the exchange current densities and transfer coefficients of reactions (5) and (6)(i.e., $i_{05}, i_{06}, \alpha_{5}$, and $\alpha_{6}$ ). Experiments of type 2 were used with to determine the kinetic parameters of reactions (2-4). Experiments of type 3 were used to estimate the exchange current density and transfer coefficient of reaction (1) (i.e., $i_{o 1}$ and $\alpha_{1}$ ). It is important to note that the kinetic parameters of reactions (2-4) were required to analyze the data of experiments of type 3 . In the full batch experiments of type 4 , a starting catholyte was electrochemically reduced to a point where $95 \%$ of the $\mathrm{NaNO}_{3}$ and $\mathrm{NaNO}_{2}$ were destroyed. The results for the experiments of type 1-3 are presented Section 2.3. Results from type 4 experiments are presented in Section 5.

The estimation of the kinetic parameters of reactions (2-4) requires the control of certain independent variables to characterize the process. Table 1A shows the experimental design for the constant-current process. The independent variables are the concentration of $\mathrm{NO}_{2}^{-}$, temperature $(\mathrm{T})$, and the current density $\left(\mathrm{i}_{1}\right)$ and they were varied using the partial $2^{3}$ factorial design. Six experimental runs will be discussed below and used for the determination of the kinetic parameters. Table $1 \mathrm{C}$ gives a summary of all parallel plate experiments performed during this project. Appendix $I$ in reference 15 contains the data for all of these experiments.

The concentrations of $\mathrm{NO}_{2}^{-}$, temperatures, and current densities were chosen to be in a suitable range for experimental and pilot plant operations. At the low temperatures $\left(25^{\circ} \mathrm{C}\right)$ the experiments with initial concentrations of $\mathrm{NO}_{2}^{-}$of $0.1 \mathrm{M}$ were not feasible because the partial current density for the reduction of water was observed to be predominating. Also, experimental data for the high temperature, high concentration, low current conditions were not obtained because preliminary experiments indicated that no additional information on selectivity could be obtained from these experiments.

Data corresponding to reasonable deviations in the instantaneous currents are shown in Tables $2 \mathrm{~A}, 2 \mathrm{~B}$ and $2 \mathrm{C}$ for the six experiments shown in Table $1 \mathrm{~A}$. For the three experiments for nitrate reduction shown in Table $1 \mathrm{~B}$, the data corresponding to reasonable deviations in the instantaneous currents are shown in Tables $3 \mathrm{~A}$ and $3 \mathrm{~B}$. The columns of Tables 2A, 2B and 3A that are labeled Delta \% Total $\mathrm{N}$ and Delta \% Total $\mathrm{OH}$ correspond to the accuracy in the analytical determination of the total nitrogen atoms and the total moles of 
$\mathrm{OH}$. The equations used to calculate these values are included in Appendix C, Section 8.3. The columns of Table $2 \mathrm{C}$ and $3 \mathrm{~B}$ labeled Delta correspond to the voltage and current balances in Volts and Amps, respectively. It is important to note that the values of Delta \% Total N, Delta \% Total $\mathrm{OH}$, and the Delta for the current include measurements of the instantaneous gas flowrates. Small accumulations of gas within the system can produce large errors in these values. Also, at some times the amount of $\mathrm{NH}_{3}$ was below the detection limit and large errors in the partial current from ammonia production are evident. These values must be viewed as a set at any given time and we have determined that the data shown in these tables are consistent. With the exception of run \# 21, the amount of unaccounted nitrogen was less than $30 \%$ at any measurement. This accuracy is probably the best that can be expected for batch experiments with this system.

The estimation of the kinetic parameters for $\mathrm{NO}_{3}{ }^{-}$reduction requires the use of the data obtained from the design shown in Table 1A. Because some of the $\mathrm{NO}_{2}^{-}$reacted in the $\mathrm{NO}_{3}{ }^{-}$ experiments, the experimental design for $\mathrm{NO}_{3}^{-}$is shown in Table 1B. Table 1B shows the three independent variables: temperature, current density and $\mathrm{NO}_{3}^{-}$concentrations. These experiments were started without $\mathrm{NO}_{2}^{-}$in an effort to simplify the analysis of $\mathrm{NO}_{2}^{-}$products. However, some of the produced $\mathrm{NO}_{2}^{-}$reacted to $\mathrm{NH}_{3}, \mathrm{~N}_{2}$, and $\mathrm{N}_{2} \mathrm{O}$ as shown in Table 3A.

Table 1A. Experimental Design for Determination of Nitrite Reduction Kinetics and Selectivity in 3.0 M NaOH Solutions. The high and low values of the independent variables are shown above the experimental run numbers.

\begin{tabular}{|c|c|c|c|c|c|c|c|c|}
\hline Temperature & \multicolumn{4}{|c|}{ Low $=25^{\circ} \mathrm{C}$} & \multicolumn{4}{|c|}{ High $=35^{\circ} \mathrm{C}$} \\
\hline Current Density & \multicolumn{2}{|c|}{$\begin{array}{l}\text { Low }=0.15 \\
\mathrm{~A} / \mathrm{cm}^{2}\end{array}$} & \multicolumn{2}{|c|}{$\begin{array}{l}\text { High }= \\
0.25 \mathrm{~A} / \mathrm{cm}^{2}\end{array}$} & \multicolumn{2}{|c|}{$\begin{array}{l}\text { Low }=0.15 \\
\mathrm{~A} / \mathrm{cm}^{2}\end{array}$} & \multicolumn{2}{|c|}{$\begin{array}{l}\text { High }=0.25 \\
\mathrm{~A} / \mathrm{cm}^{2}\end{array}$} \\
\hline $\mathrm{NO}_{2}^{-}$, Concentration & $0.1 \mathrm{M}$ & $0.6 \mathrm{M}$ & $0.1 \mathrm{M}$ & $0.6 \mathrm{M}$ & $0.1 \mathrm{M}$ & $0.6 \mathrm{M}$ & $\begin{array}{l}0.1 \\
\mathbf{M}\end{array}$ & $0.6 \mathrm{M}$ \\
\hline Run & & $\# 27$ & & $\# 34$ & $\# 20$ & & $\# 21$ & $\# 26,28$ \\
\hline
\end{tabular}

Table 1B. Experimental Design for Determination of Nitrate Reduction Kinetics in 3.0 $\mathrm{M}$ NaOH Solutions. The high and low values of the independent variables are shown above the experimental run numbers.

\begin{tabular}{|c|c|c|c|c|c|c|c|c|}
\hline Temperature & \multicolumn{4}{|c|}{ Low $=25^{\circ} \mathrm{C}$} & \multicolumn{4}{|c|}{ High $=35^{\circ} \mathrm{C}$} \\
\hline Current Density & \multicolumn{2}{|c|}{$\begin{array}{l}\text { Low }=0.15 \\
\mathrm{~A} / \mathrm{cm}^{2}\end{array}$} & \multicolumn{2}{|c|}{$\begin{array}{l}\text { High }= \\
0.25 \mathrm{~A} / \mathrm{cm}^{2}\end{array}$} & \multicolumn{2}{|c|}{$\begin{array}{l}\text { Low }=0.15 \\
\mathrm{~A} / \mathrm{cm}^{2}\end{array}$} & \multicolumn{2}{|c|}{$\begin{array}{l}\mathrm{High}=0.25 \\
\mathrm{~A} / \mathrm{cm}^{2}\end{array}$} \\
\hline $\mathrm{NO}_{3}^{-}$, Concentration & $0.6 \mathrm{M}$ & $1.9 \mathrm{M}$ & $0.6 \mathrm{M}$ & $1.9 \mathrm{M}$ & $0.6 \mathrm{M}$ & $1.9 \mathrm{M}$ & $0.6 \mathrm{M}$ & $1.9 \mathrm{M}$ \\
\hline Run & & & & \#33 & & $\# 29$ & $\# 30$ & \\
\hline
\end{tabular}


Table IC. Summary of Completed Experiments for Simulants on Planar Nickel Cathodes by Number and Run Date. (25-35 and $35^{\circ} \mathrm{C}$ Runs in Large Volume Reactor. All Other Runs in Small Volume Reactor.)

\begin{tabular}{|c|c|c|c|c|c|c|}
\hline Exp. \# & Date & $\begin{array}{l}\text { Current } \\
\left(\mathrm{A} / \mathrm{cm}^{2}\right)\end{array}$ & $\mathrm{NaNO}_{2}$ & $\begin{array}{c}\text { Compositior } \\
\mathrm{NaNO}_{2}\end{array}$ & $\mathrm{NaOH}$ & $\begin{array}{l}\text { Temp. } \\
\left({ }^{\circ} \mathrm{C}\right)\end{array}$ \\
\hline $1^{*}$ & $6 / 28 / 94$ & 0.25 & 1.9 & 0.6 & 1.33 & $25-35$ \\
\hline $2^{*}$ & $7 / 21 / 94$ & 0.25 & 1.9 & 0.6 & 1.33 & $25-35$ \\
\hline 3 & $8 / 1 / 94$ & 0.25 & 1.9 & 0.6 & 1.33 & $25-35$ \\
\hline 4 & $1 / 10 / 95$ & 0.25 & & & 1.33 & $25-35$ \\
\hline $4 a$ & $1 / 10 / 95$ & Scan & & & 1.33 & $25-35$ \\
\hline $4 b$ & $1 / 10 / 95$ & Scan & & & 1.33 & $25-35$ \\
\hline 5 & $1 / 12 / 95$ & 0.25 & 1.9 & & 1.33 & $25-35$ \\
\hline 6 & $1-27-95$ & 0.25 & & 0.6 & 1.33 & $25-35$ \\
\hline 7 & $2 / 1 / 95$ & 0.25 & & & 1.33 & $25-35$ \\
\hline $8 a^{*}$ & $2 / 15 / 95$ & Scan & 1.9 & & 1.33 & $25-35$ \\
\hline $8 b^{*}$ & $2 / 17 / 95$ & Scan & 1.9 & & 1.33 & $25-35$ \\
\hline 9 & $2 / 20 / 95$ & 0.15 & 1.9 & & 1.33 & $25-35$ \\
\hline 10 & $2 / 22 / 95$ & 0.15 & & 0.6 & 1.33 & $25-35$ \\
\hline 11 & $3 / 3 / 95$ & Scan & & 0.6 & 1.33 & $25-35$ \\
\hline 12 & $3 / 6 / 95$ & 0.15 & & 0.6 & 1.33 & $25-35$ \\
\hline 13 & $3 / 7 / 95$ & 0.25 & & 0.6 & 1.33 & $25-35$ \\
\hline 14 & $3 / 8 / 95$ & 0.25 & & 0.6 & 1.33 & $25-35$ \\
\hline 15 & $3 / 27 / 95$ & 0.25 & & 0.1 & 1.33 & $25-35$ \\
\hline 16 & $3 / 29 / 95$ & 0.15 & & 0.1 & 1.33 & $25-35$ \\
\hline $17 \mathrm{a} / \mathrm{b}$ & $4 / 5 / 95$ & 0.15 & & 0.1 & 1.33 & $25-35$ \\
\hline 18 & $4 / 10 / 95$ & 0.25 & & 0.6 & 1.33 & $25-35$ \\
\hline 19 & $4 / 12 / 95$ & 0.15 & & 0.6 & 1.33 & $25-35$ \\
\hline $20^{*}$ & $5 / 2 / 95$ & 0.15 & & 0.1 & 1.33 & $25-35$ \\
\hline $21 *$ & $5 / 3 / 95$ & 0.25 & & 0.1 & 1.33 & $25-35$ \\
\hline 22 & $5 / 23 / 95$ & 0.25 & & 0.6 & 1.33 & $25-35$ \\
\hline 23 & $5 / 24 / 95$ & 0.25 & 0.19 & & 1.33 & $25-35$ \\
\hline 24 & $5 / 26 / 95$ & 0.15 & 0.19 & & 1.33 & $25-35$ \\
\hline $25 a^{*}$ & $2 / 21 / 95$ & Scan & & & 1.33 & $25-35$ \\
\hline $25 b^{*}$ & $5 / 31 / 95$ & Scan & & & 1.33 & $25-35$ \\
\hline $25 c^{*}$ & $6 / 1 / 95$ & Scan & & & 1.33 & $25-35$ \\
\hline $26^{*}$ & $6 / 8 / 95$ & 0.25 & & 0.6 & 1.33 & $25-35$ \\
\hline $27^{*}$ & $6 / 13 / 95$ & 0.15 & & 0.6 & 1.33 & 25 \\
\hline $28^{*}$ & $6 / 16 / 95$ & 0.25 & & 0.6 & 1.33 & 35 \\
\hline $29 *$ & $6 / 14 / 95$ & 0.25 & 1.9 & & 1.33 & 35 \\
\hline $30^{*}$ & $6 / 20 / 95$ & 0.25 & 0.6 & & 1.33 & 35 \\
\hline 31 & $6 / 21 / 995$ & 0.25 & & 0.6 & 1.33 & 35 \\
\hline 32 & $6 / 26 / 95$ & 0.25 & 1.9 & & 1.33 & 35 \\
\hline $33 *$ & $6 / 28 / 95$ & 0.25 & 1.9 & & 1.33 & 25 \\
\hline 34 & $6 / 29 / 95$ & 0.25 & & 0.6 & 1.33 & 25 \\
\hline 35 & $6 / 30 / 95$ & 0.15 & 0.19 & & 1.33 & 25 \\
\hline 36 & $8 / 3 / 95$ & 0.25 & & 0.6 & 1.33 & 25 \\
\hline 37 & $9 / 28 / 95$ & $0.25-0.05$ & 1.9 & 0.6 & 1.33 & 30 \\
\hline 38 & $10 / 11 / 95$ & 025.005 & 19 & 06 & 133 & 30 \\
\hline
\end{tabular}

* Experiments used in analysis 
Table 2A. Experimental Data for Nitrite Reduction Experiment Nos. 20, 21, 27.

\begin{tabular}{|c|c|c|c|c|c|c|c|c|c|c|c|c|}
\hline Run \# & Time (min) & Temp C & [NO2] & Vol (L) & $\begin{array}{c}\text { Total Gas } \\
\text { Flow (ml/min) }\end{array}$ & $\% \mathrm{~N} 2$ & $\% \mathrm{~N} 2 \mathrm{O}$ & $\% \mathrm{H} 2$ & {$[\mathrm{NH} 3](\mathrm{aq})$} & $\begin{array}{l}\text { Cath } \\
{[\mathrm{OH}]}\end{array}$ & $\begin{array}{l}\text { Delta \% } \\
\text { Total N }\end{array}$ & $\begin{array}{c}\text { Delta \% } \\
\text { Total OH }\end{array}$ \\
\hline 20 & 0 & 20.5 & 0.134 & 4.000 & 70.59 & 0.00 & 0.00 & 0.0 & 0.000 & 1.19 & & \\
\hline 20 & 30 & 27.3 & 0.122 & 3.976 & 71.64 & 44.09 & 0.00 & 17.2 & 0.000 & 1.28 & 1.78 & 2.5 \\
\hline 20 & 60 & 29.9 & 0.112 & 4.058 & 70.59 & 32.12 & 0.00 & 42.5 & 0.000 & 1.36 & 7.36 & 3.4 \\
\hline 20 & 90 & 31.5 & 0.105 & 4.042 & 68.57 & 25.68 & 0.00 & 52.7 & 0.000 & 1.42 & 13.51 & 1.6 \\
\hline 20 & 120 & 32.0 & 0.106 & 4.025 & 70.59 & 6.75 & 0.02 & 78.4 & 0.000 & 1.49 & 21.00 & 1.4 \\
\hline 20 & 150 & 29.9 & 0.097 & 4.004 & 82.76 & 22.12 & 0.01 & 60.1 & 0.000 & 1.53 & 21.17 & 0.4 \\
\hline 20 & 180 & 34.5 & 0.088 & 4.073 & 85.71 & 10.73 & 0.05 & 75.7 & 0.000 & 1.62 & 24.89 & 2.0 \\
\hline 20 & 210 & 32.0 & 0.060 & 4.110 & 92.31 & 15.80 & 0.03 & 64.7 & 0.016 & 1.67 & 30.43 & 4.9 \\
\hline 21 & 0 & 29.2 & 0.335 & 4.000 & 126.32 & 0.00 & 0.00 & 0.0 & 0.000 & 1.45 & & \\
\hline 21 & 30 & 33.0 & 0.265 & 4.058 & 120.00 & $20.83^{\circ}$ & 0.00 & 68.7 & 0.000 & 1.56 & 21.49 & 2.6 \\
\hline 21 & 60 & 33.0 & 0.258 & 4.049 & 133.30 & 8.20 & 0.01 & 86.3 & 0.000 & 1.64 & 20.46 & 0.1 \\
\hline 21 & 90 & 33.5 & 0.201 & 4.080 & 13.14 & 3.72 & 0.01 & 91.5 & 0.000 & 1.74 & 50.82 & 0.7 \\
\hline 21 & 120 & 34.0 & 0.202 & 4.034 & 150.00 & 2.12 & 0.01 & 94.0 & 0.000 & 1.81 & 50.42 & 1.9 \\
\hline 21 & 150 & 35.0 & 0.172 & 4.059 & 153.85 & 1.06 & 0.01 & 95.4 & 0.000 & 1.90 & 74.63 & 1.8 \\
\hline 21 & 180 & 35.0 & 0.172 & 4.057 & 160.00 & 0.39 & 0.02 & 96.2 & 0.000 & 1.97 & 74.28 & 3.3 \\
\hline 21 & 210 & 35.5 & 0.174 & 4.080 & 60.00 & 0.17 & 0.02 & 95.9 & 0.000 & 2.07 & 71.16 & 2.7 \\
\hline 27 & 0 & 22.1 & 0.468 & 3.000 & 28.57 & 29.42 & 0.00 & 0.0 & 0.000 & 1.37 & & \\
\hline 27 & 30 & 24.4 & 0.442 & 3.055 & 25.81 & 10.52 & 0.00 & 4.0 & 0.000 & 1.52 & 2.87 & 10.1 \\
\hline 27 & 60 & 25.9 & 0.424 & 3.052 & 25.53 & 6.08 & 0.00 & 5.8 & 0.000 & 1.56 & 6.90 & 11.8 \\
\hline 27 & 90 & 26.4 & 0.403 & 3.071 & 25.53 & 4.15 & 0.00 & 11.5 & 0.000 & 1.71 & 11.50 & 19.6 \\
\hline 27 & 120 & 26.4 & 0.367 & 3.195 & 25.53 & 2.22 & 0.00 & 25.9 & 0.011 & 1.83 & 14.50 & 23.1 \\
\hline 27 & 150 & 26.7 & 0.352 & 3.217 & 26.09 & 7.33 & 0.00 & 33.5 & 0.023 & 1.96 & 14.84 & 23.6 \\
\hline 27 & 180 & 26.6 & 0.336 & 3.239 & 25.53 & 5.87 & 0.02 & 31.6 & 0.035 & 2.08 & 15.51 & 24.1 \\
\hline 27 & 210 & 26.8 & 0.323 & 3.248 & 36.36 & 4.41 & 1.48 & 44.8 & 0.046 & 2.10 & 15.69 & 20.4 \\
\hline 27 & 240 & 27.1 & 0.306 & 3.258 & 30.77 & 2.95 & 2.95 & 57.9 & 0.059 & 2.25 & 16.91 & 20.8 \\
\hline
\end{tabular}


Table 2B. Experimental Data for Nitrite Reduction Experiment Nos. 26, 28, 34.

\begin{tabular}{|c|c|c|c|c|c|c|c|c|c|c|c|c|}
\hline Run \# & Time $(\min )$ & Temp C & [NO2] & $\operatorname{Vol}(L)$ & $\begin{array}{c}\text { Total Gas } \\
\text { Flow (ml/min) }\end{array}$ & $\% \mathrm{~N} 2$ & $\% \mathrm{~N} 2 \mathrm{O}$ & $\% \mathrm{H} 2$ & {$[\mathrm{NH} 3](\mathrm{aq})$} & $\begin{array}{l}\text { Cath } \\
{[\mathrm{OH}]}\end{array}$ & $\begin{array}{l}\text { Delta \% } \\
\text { Total N }\end{array}$ & $\begin{array}{c}\text { Delta \% } \\
\text { Total OH } \\
\end{array}$ \\
\hline 26 & 0 & 24.6 & 0.485 & 3.000 & 27.61 & 0.00 & 0.00 & 0.0 & 0.000 & 1.41 & & \\
\hline 26 & 30 & 29.4 & 0.457 & 3.017 & 31.58 & 19.06 & 0.00 & 1.2 & 0.000 & 1.53 & 2.17 & 1.9 \\
\hline 26 & 60 & 33.3 & 0.409 & 3.096 & 41.38 & 10.03 & 0.00 & 60.0 & 0.025 & 1.83 & 7.15 & 13.5 \\
\hline 26 & 90 & 33.9 & 0.391 & 3.108 & 46.15 & 7.39 & 0.00 & 70.4 & 0.039 & 1.92 & 7.19 & 10.7 \\
\hline 26 & 120 & 34.0 & 0.359 & 3.200 & 37.50 & 1.59 & 0.00 & 71.9 & 0.049 & 2.04 & 9.85 & 12.9 \\
\hline 26 & 150 & 34.4 & 0.330 & 3.276 & 42.86 & 3.70 & 0.02 & 75.1 & 0.072 & 2.21 & 9.00 & 12.5 \\
\hline 26 & 180 & 34.7 & 0.287 & 3.298 & 46.15 & 2.93 & 0.03 & 78.7 & 0.096 & 2.40 & 15.72 & 11.6 \\
\hline 26 & 210 & 34.8 & 0.251 & 3.452 & 60.00 & 4.02 & 0.05 & 84.5 & 0.124 & 2.67 & 13.04 & 14.3 \\
\hline 26 & 240 & 34.9 & 0.214 & 3.463 & 70.59 & 2.72 & 0.05 & 87.8 & 0.139 & 2.82 & 23.85 & 13.5 \\
\hline 28 & 0 & 32.0 & 0.438 & 3.000 & 23.53 & 12.09 & 0.00 & 0.0 & 0.000 & 1.38 & & \\
\hline 28 & 30 & 34.0 & 0.412 & 3.093 & 25.53 & 29.03 & 0.00 & 0.0 & 0.000 & 1.43 & 2.18 & 4.9 \\
\hline 28 & 60 & 36.0 & 0.395 & 3.133 & 26.97 & 25.25 & 0.01 & 0.0 & 0.000 & 1.59 & 3.62 & 14.0 \\
\hline 28 & 90 & 35.5 & 0.371 & 3.172 & 26.09 & 18.19 & 0.03 & 0.0 & 0.000 & 1.73 & 7.09 & 20.9 \\
\hline 28 & 120 & 36.0 & 0.363 & 3.104 & 27.27 & 15.86 & 0.12 & 0.0 & 0.013 & 1.80 & 6.32 & 16.6 \\
\hline 28 & 150 & 38.0 & 0.341 & 3.142 & 24.49 & 8.60 & 0.22 & 2.2 & 0.022 & 1.90 & 8.15 & 17.9 \\
\hline 28 & 180 & 38.0 & 0.317 & 3.185 & 30.00 & 40.91 & 0.51 & 5.6 & 0.046 & 2.24 & 5.83 & 22.7 \\
\hline 28 & 210 & 37.0 & 0.299 & 3.275 & 32.43 & 23.71 & 0.78 & 8.9 & 0.054 & 2.37 & 3.71 & 24.6 \\
\hline 28 & 240 & 37.0 & 0.278 & 3.276 & 47.06 & 36.17 & 1.36 & 19.5 & 0.072 & 2.50 & 2.07 & 21.3 \\
\hline 34 & 0 & 22.5 & 0.467 & 3.000 & 27.9 & 16.28 & 0.02 & 0.0 & 0.000 & 1.36 & & \\
\hline 34 & 30 & 27.1 & 0.435 & 3.098 & 26.1 & 4.29 & 0.00 & 2.9 & 0.000 & 1.53 & 3.35 & 13.2 \\
\hline 34 & 60 & 29.3 & 0.399 & 3.056 & 24.5 & 11.23 & 0.01 & 1.5 & 0.000 & 1.69 & 13.48 & 20.0 \\
\hline 34 & 90 & 30.6 & 0.376 & 3.185 & 28.6 & 15.68 & 0.01 & 6.2 & 0.035 & 1.83 & 5.60 & 15.0 \\
\hline 34 & 120 & 31.3 & 0.345 & 3.245 & 34.3 & 14.55 & 0.01 & 22.5 & 0.090 & 1.98 & 4.03 & 2.1 \\
\hline 34 & 150 & 31.6 & 0.318 & 3.280 & 33.3 & 8.04 & 0.03 & 34.2 & 0.101 & 2.18 & 1.98 & 7.2 \\
\hline 34 & 180 & 32.1 & 0.282 & 3.431 & 38.7 & 9.84 & 0.05 & 60.4 & 0.101 & 2.24 & 3.34 & 11.1 \\
\hline 34 & $210^{\circ}$ & 32.3 & 0.255 & 3.458 & 46.2 & 7.41 & 0.07 & 76.8 & 0.115 & 2.39 & 6.84 & 11.5 \\
\hline 34 & 240 & 32.7 & 0.235 & 3.505 & 63.2 & 8.78 & 0.06 & 78.2 & 0.139 & 2.55 & 1.94 & 9.6 \\
\hline
\end{tabular}


Table 2C. Experimental Voltages and Partial Currents for Nitrite Reduction Experiments.

\begin{tabular}{|c|c|c|c|c|c|c|c|c|c|c|c|c|}
\hline \multirow[b]{2}{*}{ Run \# } & \multirow[b]{2}{*}{ Time (min) } & \multirow[b]{2}{*}{ Current (A) } & \multicolumn{5}{|c|}{ Potential Balance (Volts) } & \multicolumn{5}{|c|}{ Instantaneous Current (A) } \\
\hline & & & Cell & Anode & Membrane & Cathode & Delta & N2 & $\mathrm{N} 2 \mathrm{O}$ & NH3 & $\mathrm{H} 2$ & Delta \\
\hline 20 & 120 & 15.086 & 3.636 & 0.825 & 0.777 & 2.036 & -0.002 & 2.054 & 0.004 & 0.000 & 7.952 & 3.018 \\
\hline 20 & 150 & 15.089 & 3.671 & 0.817 & 0.784 & 2.086 & 0.002 & 7.885 & 0.001 & 0.000 & 7.142 & 0.061 \\
\hline 20 & 180 & 15.091 & 3.713 & 0.802 & 0.806 & 2.105 & 0.000 & 3.962 & 0.011 & 0.000 & 9.318 & 1.800 \\
\hline 20 & 210 & 15.070 & 3.746 & 0.808 & 0.805 & 2.131 & 0.002 & 6.282 & 0.007 & 21.153 & 8.579 & -20.951 \\
\hline 21 & 120 & 24.778 & 4.592 & 1.011 & 1.276 & 2.303 & 0.002 & 1.367 & 0.005 & 0.000 & 20.255 & 3.151 \\
\hline 21 & 150 & 24.818 & 4.654 & 0.970 & 1.346 & 2.331 & -0.002 & 0.705 & 0.005 & 0.000 & 21.067 & 3.040 \\
\hline 21 & 180 & 24.797 & 4.692 & 1.001 & 1.337 & 2.354 & 0.000 & 0.266 & 0.008 & 0.000 & 22.102 & 2.421 \\
\hline 21 & 210 & 24.724 & 4.752 & 1.027 & 1.361 & 2.369 & -0.005 & 0.045 & 0.004 & 0.000 & 8.263 & 16.413 \\
\hline 26 & 150 & 24.359 & 4.329 & 2.513 & 0.003 & 1.812 & 0.001 & 0.683 & 0.003 & 25.954 & 4.622 & -6.904 \\
\hline 26 & 180 & 24.372 & 4.339 & 2.533 & 0.006 & 1.802 & -0.002 & 0.583 & 0.004 & 25.441 & 5.215 & -6.871 \\
\hline 26 & 210 & 24.391 & 4.378 & 2.589 & 0.043 & 1.821 & -0.075 & 1.038 & 0.009 & 35.610 & 7.280 & -19.546 \\
\hline 26 & 240 & 24.404 & 4.426 & 2.640 & 0.054 & 1.837 & -0.104 & 0.828 & 0.010 & 17.926 & 8.900 & -3.258 \\
\hline 27 & 180 & 15.006 & 3.661 & 0.867 & 0.905 & 1.885 & 0.004 & 0.646 & 0.002 & 12.457 & 1.160 & 0.742 \\
\hline 27 & 210 & 15.008 & 3.714 & 0.845 & 0.946 & 1.923 & 0.000 & 0.691 & 0.155 & 12.220 & 2.339 & -0.397 \\
\hline 27 & 240 & 15.007 & 3.824 & 0.863 & 0.916 & 2.062 & -0.017 & 0.391 & 0.261 & 13.564 & 2.558 & -1.766 \\
\hline 28 & 150 & 25.117 & 4.212 & 1.016 & 1.183 & 2.007 & 0.006 & 0.908 & 0.016 & 9.406 & 0.079 & 14.709 \\
\hline 28 & 180 & 25.100 & 4.231 & 1.166 & 0.780 & 2.264 & 0.021 & 5.288 & 0.044 & 24.185 & 0.241 & -4.658 \\
\hline 28 & 210 & 24.922 & 4.253 & 1.035 & 1.071 & 2.144 & 0.003 & 3.313 & 0.073 & 10.029 & 0.414 & 11.094 \\
\hline 28 & 240 & 24.940 & 4.371 & 1.052 & 1.105 & 2.204 & 0.010 & 7.332 & 0.183 & 19.453 & 1.318 & -3.347 \\
\hline 34 & 150 & 31.6 & 0.318 & 3.280 & 33.3 & 8.04 & 0.03 & 34.2 & 0.101 & 2.18 & 1.98 & 7.2 \\
\hline 34 & 180 & 32.1 & 0.282 & 3.431 & 38.7 & 9.84 & 0.05 & 60.4 & 0.101 & 2.24 & 3.34 & 11.1 \\
\hline 34 & 210 & 32.3 & 0.255 & 3.458 & 46.2 & 7.41 & 0.07 & 76.8 & 0.115 & 2.39 & 6.84 & 11.5 \\
\hline 34 & 240 & 32.7 & 0.235 & 3.505 & 63.2 & 8.78 & 0.06 & 78.2 & 0.139 & 2.55 & 1.94 & 9.6 \\
\hline
\end{tabular}


Table 3A. Experimental Data for Nitrate Reduction Experiments.

\begin{tabular}{|c|c|c|c|c|c|c|c|c|c|c|c|c|c|}
\hline Run \# & Time (min) & Temp C & [NO3] & [NO2] & $\operatorname{Vol}(\mathrm{L})$ & $\begin{array}{c}\text { Total Gas } \\
\text { Flow (ml/min) }\end{array}$ & $\% \mathrm{~N} 2$ & $\% \mathrm{~N} 2 \mathrm{O}$ & $\% \mathrm{H} 2$ & {$[\mathrm{NH} 3](\mathrm{aq})$} & $\begin{array}{l}\text { Cath } \\
{[\mathrm{OH}]}\end{array}$ & $\begin{array}{l}\text { Delta \% } \\
\text { Total N }\end{array}$ & $\begin{array}{c}\text { Delta \% } \\
\text { Total } \mathrm{OH}\end{array}$ \\
\hline 29 & 0 & 34.5 & 1.620 & 0.000 & 4.000 & 27.91 & 9.74 & 0.00 & 0.0 & 0.000 & 1.59 & & \\
\hline 29 & 30 & 34.0 & 1.552 & 0.005 & 4.138 & 30.00 & 17.28 & 0.00 & 0.0 & 0.000 & 1.73 & 0.41 & 9.0 \\
\hline 29 & 60 & 34.0 & 1.567 & 0.019 & 4.169 & 31.58 & 24.97 & 0.01 & 0.0 & 0.000 & 1.80 & 2.40 & 15.2 \\
\hline 29 & 90 & 36.0 & 1.538 & 0.023 & 4.222 & 24.24 & 1.60 & 0.04 & 0.0 & 0.000 & 1.91 & 2.30 & 19.5 \\
\hline 29 & 120 & 38.0 & 1.505 & 0.029 & 4.284 & 27.27 & 11.91 & 0.06 & 1.7 & 0.000 & 2.02 & 2.06 & 23.7 \\
\hline 29 & 150 & 38.0 & 1.504 & 0.032 & 4.335 & 23.08 & 10.86 & 0.07 & 4.0 & 0.000 & 2.09 & 3.50 & 28.4 \\
\hline 29 & 180 & 39.0 & 1.481 & 0.043 & 4.355 & 28.57 & 9.80 & 0.12 & 3.4 & 0.016 & 2.18 & 4.29 & 24.8 \\
\hline 29 & 210 & 37.0 . & 1.441 & 0.064 & 4.387 & 28.92 & 4.14 & 0.26 & 9.9 & 0.021 & 2.19 & 4.25 & 20.9 \\
\hline 29 & 240 & 40.5 & 1.391 & 0.090 & 4.486 & 25.53 & 0.00 & 0.59 & 16.3 & 0.021 & 2.39 & 4.90 & 27.4 \\
\hline 30 & 0 & 30.0 & 0.480 & 0.016 & 4.000 & 171.40 & 77.17 & 0.02 & 0.0 & 0.000 & 1.37 & & \\
\hline 30 & 30 & 35.0 & 0.472 & 0.017 & 3.866 & 85.71 & 33.97 & 0.01 & 46.2 & 0.000 & 1.49 & 6.49 & 17.4 \\
\hline 30 & 60 & 37.0 & 0.458 & 0.018 & 3.948 & 82.76 & 14.28 & 0.01 & 69.3 & 0.000 & 1.57 & 8.85 & 15.3 \\
\hline 30 & 90 & 35.0 & 0.437 & 0.023 & 3.993 & 75.00 & 8.85 & 0.01 & 77.0 & 0.000 & 1.68 & 8.09 & 12.6 \\
\hline 30 & 120 & 37.0 & 0.424 & 0.023 & 3.993 & 75.00 & 12.91 & 0.01 & 72.9 & 0.010 & 1.80 & 9.29 & 14.6 \\
\hline 30 & 150 & 36.0 & 0.407 & 0.026 & 3.994 & 70.59 & 11.18 & 0.01 & 71.0 & 0.020 & 1.83 & 10.63 & 22.5 \\
\hline 30 & 180 & 37.0 & 0.387 & 0.026 & 3.963 & 70.59 & 5.13 & 0.00 & 81.2 & 0.030 & 1.96 & 8.16 & 22.8 \\
\hline 30 & 210 & 37.0 & 0.385 & 0.026 & 3.976 & 68.57 & 12.07 & 0.00 & 69.6 & 0.040 & 2.05 & 11.55 & 23.3 \\
\hline 30 & 240 & 38.0 & 0.345 & 0.028 & 3.892 & 75.00 & 9.25 & 0.00 & 71.4 & 0.040 & 2.13 & 1.79 & 28.2 \\
\hline 33 & 0 & 24.1 & 1.856 & 0.009 & 3.000 & 26.09 & 18.27 & 0.00 & 0.0 & 0.000 & 1.40 & & \\
\hline 33 & 30 & 27.7 & 1.789 & 0.016 & 3.090 & 23.08 & 14.37 & 0.00 & 0.0 & 0.000 & 1.74 & 0.12 & 19.6 \\
\hline 33 & 60 & 28.9 & 1.778 & 0.017 & 3.111 & 23.30 & 16.03 & 0.00 & 0.0 & 0.000 & 1.81 & 0.17 & 22.7 \\
\hline 33 & 90 & 29.7 & 1.764 & 0.019 & 3.127 & 23.53 & 17.57 & 0.00 & 0.5 & 0.020 & 1.88 & 1.32 & 17.2 \\
\hline 33 & 120 & 30.1 & 1.635 & 0.021 & 3.335 & 22.86 & 16.81 & 0.00 & 1.6 & 0.030 & 1.98 & 1.25 & 19.8 \\
\hline 33 & 150 & 30.3 & 1.608 & 0.024 & 3.356 & 22.86 & 15.27 & 0.00 & 3.1 & 0.040 & 2.12 & 1.23 & 20.0 \\
\hline 33 & 180 & 30.1 & 1.598 & 0.028 & 3.369 & 24.00 & 16.88 & 0.00 & 3.7 & 0.060 & 2.24 & 2.68 & 17.4 \\
\hline 33 & 210 & 30.4 & 1.568 & 0.030 & 3.393 & 23.76 & 20.94 & 0.00 & 4.0 & 0.070 & 2.37 & 2.55 & 17.2 \\
\hline 33 & 240 & 30.6 & 1.529 & 0.032 & 3.459 & 24.49 & 11.91 & 0.05 & 4.3 & 0.090 & 2.50 & 3.70 & 15.8 \\
\hline
\end{tabular}


Table 3B. Experimental Voltages and Partial Currents for Nitrate Reduction Experiments.

\begin{tabular}{|c|c|c|c|c|c|c|c|c|c|c|c|c|c|}
\hline \multirow[b]{2}{*}{ Run \# } & \multirow[b]{2}{*}{ Time (min) } & \multirow[b]{2}{*}{ Current (A) } & \multicolumn{5}{|c|}{ Potential Balance } & \multicolumn{6}{|c|}{ Instantaneous Current } \\
\hline & & & Cell & Anode & Membrane & Cathode & Delta & $\mathrm{N} 2$ & $\mathrm{~N} 2 \mathrm{O}$ & NH3 & $\mathrm{H} 2$ & NO2- & Delta \\
\hline 29 & 0 & 25.182 & 4.364 & 0.936 & 1.422 & 2.006 & 0.000 & 1.171 & 0 & 0 & 0 & 1.171 & 22.84 \\
\hline 29 & 30 & 25.134 & 4.286 & 0.929 & 1.345 & 2.014 & -0.002 & 2.233 & 0.000 & 0.000 & 0.000 & 6.160 & 16.741 \\
\hline 29 & 60 & 25.088 & 4.277 & 0.925 & 1.225 & 2.123 & 0.004 & 3.397 & 0.001 & 0.000 & 0.000 & 3.398 & 18.292 \\
\hline 29 & 90 & 25.088 & 4.149 & 0.921 & 1.291 & 1.940 & -0.003 & 0.168 & 0.003 & 0.000 & 0.000 & 4.224 & 20.693 \\
\hline 29 & 120 & 24.965 & 4.110 & 0.922 & 1.266 & 1.921 & 0.001 & 1.400 & 0.005 & 0.000 & 0.066 & 4.843 & 18.652 \\
\hline 29 & 150 & 24.944 & 4.158 & 0.928 & 1.171 & 2.067 & -0.008 & 1.080 & 0.004 & 0.000 & 0.132 & 1.084 & 22.644 \\
\hline 29 & 180 & 24.940 & 4.241 & 0.919 & 1.192 & 2.131 & -0.001 & 1.207 & 0.010 & 21.993 & 0.140 & 7.514 & -5.924 \\
\hline 29 & 210 & 24.946 & 4.268 & 0.922 & 1.901 & 2.140 & -0.695 & 0.515 & 0.021 & 8.064 & 0.411 & 13.781 & 2.153 \\
\hline 29 & 240 & 24.969 & 4.284 & 0.925 & 1.226 & 2.138 & -0.005 & 0.000 & 0.044 & 0.101 & 0.599 & 8.658 & 15.568 \\
\hline 30 & 0 & 25.000 & 4.640 & 1.150 & 1.050 & 1.400 & 0.040 & 56.981 & 0.008 & 0.000 & 0.000 & 56.989 & -88.978 \\
\hline 30 & 30 & 25.000 & 4.500 & 1.200 & 0.920 & 2.390 & 0.020 & 12.542 & 0.002 & 0.000 & 5.684 & 12.544 & -5.772 \\
\hline 30 & 60 & 25.000 & 4.420 & 0.965 & 1.350 & 2.080 & 0.025 & 5.092 & 0.002 & 0.000 & 8.238 & 5.094 & 6.574 \\
\hline 30 & 90 & 25.000 & 4.350 & 0.960 & 1.360 & 2.030 & 0.000 & 2.860 & 0.002 & 0.000 & 8.290 & 6.781 & 7.067 \\
\hline 30 & 120 & 25.000 & 4.330 & 1.050 & 1.220 & 2.060 & 0.000 & 4.173 & 0.003 & 15.195 & 7.846 & 6.708 & -8.923 \\
\hline 30 & 150 & 25.000 & 4.320 & 0.965 & 1.360 & 1.980 & 0.015 & 3.401 & 0.001 & 16.102 & 7.201 & 7.235 & -8.941 \\
\hline 30 & 180 & 25.000 & 4.310 & 0.980 & 1.360 & 1.980 & -0.010 & 1.561 & 0.001 & 8.629 & 8.233 & 9.851 & -3.275 \\
\hline 30 & 210 & 25.000 & 4.320 & 1.000 & 1.360 & 1.970 & -0.010 & 3.566 & 0.001 & 13.483 & 6.851 & 5.814 & -4.715 \\
\hline 30 & 240 & 25.000 & 4.330 & 1.040 & 1.310 & 1.980 & 0.000 & 2.989 & 0.001 & -0.257 & 7.691 & 20.160 & -5.583 \\
\hline 33 & 0 & 24.596 & 4.795 & 1.092 & 1.652 & 2.039 & 0.012 & 2.053 & 0.000 & 0.000 & 0.000 & 2.053 & 20.489 \\
\hline 33 & 30 & 24.424 & 4.569 & 1.081 & 1.488 & 2.004 & -0.004 & 1.429 & 0.000 & 0.000 & 0.000 & 4.288 & 18.707 \\
\hline 33 & 60 & 24.443 & 4.512 & 1.069 & 1.446 & 1.997 & 0.000 & 1.609 & 0.000 & 0.000 & 0.000 & 1.609 & 21.225 \\
\hline 33 & 90 & 24.435 & 4.483 & 1.061 & 1.392 & 2.023 & 0.007 & 1.781 & 0.000 & 20.117 & 0.017 & 5.134 & -2.614 \\
\hline 33 & 120 & 24.435 & 4.489 & 1.056 & 1.380 & 2.054 & -0.001 & 1.655 & 0.000 & 12.066 & 0.053 & 6.787 & 3.874 \\
\hline 33 & 150 & 24.449 & 4.489 & 1.069 & 1.383 & 2.037 & 0.000 & 1.504 & 0.000 & 10.998 & 0.102 & 6.034 & 5.811 \\
\hline 33 & 180 & 24.460 & 4.477 & 1.214 & 1.264 & 2.000 & -0.001 & 1.745 & 0.000 & 21.841 & 0.128 & 5.385 & -4.639 \\
\hline 33 & 210 & 24.464 & 4.492 & 1.224 & 1.229 & 2.037 & 0.002 & 2.143 & 0.000 & 11.377 & 0.136 & 6.802 & 4.005 \\
\hline 33 & 240 & 24.493 & 4.510 & 1.237 & 1.231 & 2.042 & 0.000 & 1.257 & 0.004 & 23.739 & 0.151 & 5.217 & -5.874 \\
\hline
\end{tabular}




\subsection{Results and Discussion}

The results of experiments of type 2, shown in Tables $2 \mathrm{~A}, 2 \mathrm{~B}$, and $2 \mathrm{C}$ were used to determine the exchange current densities and transfer coefficients for the reduction of $\mathrm{NO}_{2}^{-}$ according to reactions (2-4). These experiments were performed at current densities of 0.15 and $0.25 \mathrm{~A} / \mathrm{cm}^{2}$, at temperatures of 25 and $35^{\circ} \mathrm{C}$, and initial $\mathrm{NO}_{2}{ }^{-}$concentrations of 0.1 and $0.6 \mathrm{M}$. Transient changes in the concentrations of $\mathrm{NaOH}$ and $\mathrm{NaNO}_{2}$ were monitored at onehalf hour intervals via titration for $\mathrm{OH}^{-}$and ion chromatography for $\mathrm{NO}_{2}^{-}$. The catholyte volume, temperature, and potential drops in the electrochemical cell were measured at one-half hour intervals. The off-gas composition and molar flow rates were determined by gas chromatography (GC).

In these experiments, the $\mathrm{NH}_{3}$ remained in solution at dilute concentrations while $\mathrm{H}_{2}$, $\mathrm{N}_{2}$ and $\mathrm{N}_{2} \mathrm{O}$ were detected and measured by $\mathrm{GC}$ analysis in the off-gas. This was due to a small Henry's law coefficient for $\mathrm{NH}_{3}$ (i.e., $\mathrm{H}\left(\mathrm{NH}_{3}\right)=765 \mathrm{~mm} \mathrm{Hg} @ 25^{\circ} \mathrm{C}$, see Appendix D of Wingard [15] for the predictions determined by experimental data and ASPEN Plus). Therefore, a Kjeldahl analysis was necessary to determine the liquid phase concentration of $\mathrm{NH}_{3}$ and $\mathrm{NH}_{4}{ }^{+}$in the catholyte.

The instantaneous off-gas flow rate was measured at 30-minute intervals, using an inverted $50 \mathrm{ml}$ burette as a bubble meter. This was used as a check of the off-gas flow rates determined from the GC analysis of the off-gas and argon carrier. The material balance was closed by a species and overall mass balance on the reacting species in the catholyte, the flux of water through the membrane, and the catholyte off-gas. Closure of the material balance was to within $10 \%$ in most cases and is outlined below. An instantaneous current balance is included in Tables $2 \mathrm{~A}, 2 \mathrm{~B}$, and $2 \mathrm{C}$ as a check of the overall material balance.

The results of experiments of type 3 , shown in Tables $3 \mathrm{~A}$ and $3 \mathrm{~B}$, were used to determine the exchange current density $\left(i_{1}\right)$ and transfer coefficient $\left(\alpha_{1}\right)$ for the reduction of $\mathrm{NO}_{3}{ }^{-}$according to reaction 1 . The experiments were performed under pseudo steady-state conditions at current densities of 0.15 and $0.25 \mathrm{~A} / \mathrm{cm}^{2}$ and concentrations of approximately 0.6 and $1.3 \mathrm{M} \mathrm{NO}_{3}^{-}$. Starting and transient solution concentrations were analyzed via titration for $\mathrm{OH}^{-}$and ion chromatography for $\mathrm{NO}_{3}^{-}$and $\mathrm{NO}_{2}^{-}$. The catholyte volume, temperature, and potential drops in the electrochemical cell were measured at one-half hour intervals. Off-gas flow from the catholyte was monitored during the experiment, but the off-gas flow rate, at the starting concentrations of 0.6 and $1.3 \mathrm{M} \mathrm{NO}_{3}^{-}$, was not sufficient to quantify the reduction products of the $\mathrm{NO}_{2}{ }^{-}$or $\mathrm{H}_{2} \mathrm{O}$ reactions. Kjeldahl analysis at the end of the experiments indicated the presence of $\mathrm{NH}_{3}$. The concentration of $\mathrm{NO}_{3}{ }^{-}$was observed to decline over the short duration of the experiment and this corresponded to an increase in the $\mathrm{NO}_{2}^{-}$ concentration.

In the batch experiments of type 1, the anolyte and catholyte were titrated to determine the concentrations of $\mathrm{NaOH}$ before and after the experiment, and the temperature was measured throughout. Four voltage differences were measured at each corresponding current density as discussed above; (1) the potential difference between the anode and the anode reference electrode, (2) the difference between the cathode reference electrode and the cathode, (3) the difference between the cathode and anode reference electrodes, and (4) the total potential difference between the anode and cathode. It was important to allow the system 
to reach steady-state so that the reference potential measurements were reproducible. Therefore, a Princeton Applied Research Model 273A was used to run consecutive chronopotentiometry experiments in which the applied current density was held at each step for 15 minutes between $1.0 \times 10^{-4}$ and $1.0 \times 10^{-2} \mathrm{~A} / \mathrm{cm}^{2}$. A Lambda regulated power supply was then used to make current density steps from $1.0 \times 10^{-2}$ to $0.25 \mathrm{~A} / \mathrm{cm}^{2}$.

The current-potential data for experiments $25 \mathrm{~b}$ and $25 \mathrm{c}$ are shown in Tables 4 and 5.These data must be corrected for the IR drop between the reference electrode and either the anode or cathode. The catholyte resistance was determined using linear regression and found to be 0.0272 and $0.0231 \Omega$ for experiments $25 \mathrm{~b}$ and $25 \mathrm{c}$, respectively. The anolyte resistance was 0.03279 and $0.02697 \Omega$ for experiments $25 \mathrm{~b}$ and $25 \mathrm{c}$, respectively. Tafel plots are shown in Figures 4 and 5. The exchange current densities and the transfer coefficients for the reduction of water at a nickel cathode and the oxidation of $\mathrm{OH}^{-}$at a stainless steel anode were determined by Tafel analyses. The results of these analyses are shown in Table 6. 
Table 4. Experiment 25b Current-Potential Data for the Reduction of $\mathrm{H}_{2} \mathrm{O}$ and the Oxidation of $\mathrm{OH}^{-}$.

\begin{tabular}{|c|c|c|c|c|c|}
\hline \multicolumn{6}{|c|}{ Electrode Materials: Nickel Cathode, Stainless Steel Anode; Date: 05/31/95 } \\
\hline \multicolumn{6}{|c|}{ Electrolyte Conc.: $2.09 \mathrm{M} \mathrm{NaOH}$ Catholyte and $1.99 \mathrm{M} \mathrm{NaOH}$ Anolyte } \\
\hline & $\begin{array}{c}\text { Measured } \\
\text { Anode vs } \\
\text { Cathode (V) } \\
\end{array}$ & $\begin{array}{c}\text { Anode vs } \\
\text { Anode Ref. } \\
\text { (V) }\end{array}$ & $\begin{array}{c}\text { Cathode vs } \\
\text { Cathode Ref. } \\
\text { (V) }\end{array}$ & $\begin{array}{c}\text { Anode Ref. vs } \\
\text { Cathode Ref. } \\
\text { (V) }\end{array}$ & Exp. Error (V) \\
\hline Open Circuit & 0.00135 & -0.2680 & -0.2751 & 0.006345 & 0.0121 \\
\hline \multicolumn{6}{|l|}{$\begin{array}{c}\text { Current Density } \\
\left(\mathrm{A} / \mathrm{cm}^{2}\right)\end{array}$} \\
\hline $1.0 \times 10^{-5}$ & 0.9469 & 0.2805 & -0.6636 & 0.006175 & 0.0034 \\
\hline $5.0 \times 10^{-5}$ & 1.243 & 0.4163 & -0.8202 & 0.00616 & -0.00034 \\
\hline $1.0 \times 10^{-4}$ & 1.325 & 0.4509 & -0.8900 & 0.005876 & 0.0218 \\
\hline $2.0 \times 10^{-4}$ & 1.513 & 0.4378 & -1.067 & 0.00643 & -0.00177 \\
\hline $5.0 \times 10^{-4}$ & 1.682 & 0.4742 & -1.193 & 0.009952 & -0.00485 \\
\hline $1.0 \times 10^{-3}$ & 1.769 & 0.4612 & -1.284 & 0.01447 & -0.00933 \\
\hline $2.0 \times 10^{-3}$ & 1.849 & 0.4722 & -1.358 & 0.01942 & 0.00062 \\
\hline $5.0 \times 10^{-3}$ & 1.933 & 0.5110 & -1.402 & 0.03800 & 0.0018 \\
\hline $7.5 \times 10^{-3}$ & 1.987 & 0.5059 & -1.428 & 0.05374 & 0.00064 \\
\hline $9.0 \times 10^{-3}$ & 2.015 & 0.5127 & -1.440 & 0.06322 & 0.00092 \\
\hline 0.01 & 2.003 & 0.5158 & -1.421 & 0.06843 & 0.00223 \\
\hline 0.02 & 2.196 & 0.5493 & -1.494 & 0.1542 & 0.0015 \\
\hline 0.05 & 2.637 & 0.6285 & -1.642 & 0.3699 & 0.0034 \\
\hline 0.10 & 3.152 & 0.7469 & -1.838 & 0.7211 & 0.154 \\
\hline 0.15 & 3.859 & 0.8621 & -1.990 & 1.055 & 0.0481 \\
\hline 0.20 & 4.489 & 0.9710 & -2.149 & 1.366 & -0.003 \\
\hline 0.25 & 4.971 & 1.753 & -2.278 & 1.616 & 0.676 \\
\hline
\end{tabular}


Table 5. Experiment 25c Current-Potential Data for the Reduction of $\mathrm{H}_{2} \mathrm{O}$ and the Oxidation of $\mathrm{OH}$.

\begin{tabular}{|c|c|c|c|c|c|}
\hline \multicolumn{4}{|c|}{ Electrode Materials: Nickel Cathode, Stainless Steel Anode } & \multicolumn{2}{|c|}{ Date: 06/01/95 } \\
\hline \multicolumn{6}{|c|}{ Electrolyte Conc.: $2.12 \mathrm{M} \mathrm{NaOH}$ Catholyte and $1.81 \mathrm{M} \mathrm{NaOH}$ Anolyte } \\
\hline & $\begin{array}{c}\text { Measured } \\
\text { Anode vs } \\
\text { Cathode (V) }\end{array}$ & $\begin{array}{l}\text { Anode vs } \\
\text { Anode Ref. } \\
\text { (V) }\end{array}$ & $\begin{array}{l}\text { Cathode vs } \\
\text { Cathode Ref. } \\
\text { (V) }\end{array}$ & $\begin{array}{c}\text { Anode Ref. } \\
\text { vs Cathode } \\
\text { Ref. (V) }\end{array}$ & Exp. Error (V) \\
\hline Open Circuit & 0.1363 & -0.2684 & -0.3897 & 0.01184 & -0.00316 \\
\hline \multicolumn{6}{|l|}{$\begin{array}{l}\text { Current Density } \\
\left(\mathrm{A} / \mathrm{cm}^{2}\right)\end{array}$} \\
\hline $1.0 \times 10^{-5}$ & 1.031 & 0.2980 & -0.7219 & 0.01364 & 0.00254 \\
\hline $5.0 \times 10^{-5}$ & 1.308 & 0.4225 & -0.8719 & 0.01268 & -0.00092 \\
\hline $1.0 \times 10^{-4}$ & 1.389 & 0.4342 & -0.9414 & 0.01281 & -0.00059 \\
\hline $2.0 \times 10^{-4}$ & 1.556 & 0.4436 & -1.099 & 0.01318 & -0.00022 \\
\hline $5.0 \times 10^{-4}$ & 1.669 & 0.4561 & -1.197 & 0.01586 & -0.00004 \\
\hline $1.0 \times 10^{-3}$ & 1.732 & 0.4666 & -1.246 & 0.01961 & 0.00021 \\
\hline $2.0 \times 10^{-3}$ & 1.798 & 0.4791 & -1.292 & 0.02638 & -0.00052 \\
\hline $5.0 \times 10^{-3}$ & 1.914 & 0.5014 & -1.367 & 0.04753 & 0.00193 \\
\hline $7.5 \times 10^{-3}$ & 1.989 & 0.5169 & -1.410 & 0.06129 & -0.00081 \\
\hline $9.0 \times 10^{-3}$ & 2.027 & 0.5236 & -1.430 & 0.07390 & 0.0005 \\
\hline 0.01 & 2.056 & 0.5278 & -1.447 & 0.08057 & -0.00063 \\
\hline 0.02 & 2.298 & 0.5621 & -1.578 & 0.1553 & -0.0026 \\
\hline 0.05 & 2.830 & 0.6569 & -1.813 & 0.3610 & 0.0009 \\
\hline 0.10 & 3.476 & 0.8035 & -1.977 & 0.6703 & -0.0252 \\
\hline 0.15 & 4.084 & 0.9291 & -2.128 & 1.026 & -0.0009 \\
\hline 0.20 & 4.653 & 1.071 & -2.246 & 1.333 & -0.0030 \\
\hline 0.25 & 5.109 & 1.184 & -2.327 & 1.600 & 0.0020 \\
\hline
\end{tabular}

Table 6. Summary of Exchange Current Densities and Transfer Coefficients of Reactions (5) and (6) with 95\% Confidence Intervals.

\begin{tabular}{||ccccc|}
\hline Experiment No. & $\begin{array}{c}i_{6 s} \\
\left(\mathrm{~A} / \mathrm{cm}^{2}\right)\end{array}$ & $\begin{array}{c}i_{i_{6}} \\
\left(\mathrm{~A} / \mathrm{cm}^{2}\right)\end{array}$ & $\alpha_{5}$ & $\alpha_{6}$ \\
\hline $25 \mathrm{~b}$ & $1.0 \times 10^{-6} \pm 5.5 \times 10^{-9}$ & $2.5 \times 10^{-4} \pm 3.9 \times 10^{-6}$ & $0.67 \pm 0.0037$ & $0.18 \pm 0.0028$ \\
$25 \mathrm{c}$ & $1.0 \times 10^{-6} \pm 7.7 \times 10^{-9}$ & $2.0 \times 10^{-4} \pm 6.3 \times 10^{-6}$ & $0.60 \pm 0.0046$ & $0.20 \pm 0.0063$ \\
Mean & $1.0 \times 10^{-6} \pm 6.6 \times 10^{-9}$ & $2.25 \times 10^{-4} \pm 5.1 \times 10^{-6}$ & $0.635 \pm 0.0042$ & $0.19 \pm 0.0046$ \\
\hline
\end{tabular}


Figure 4 : Tafel Plot for the Anodic Oxidation of $\mathrm{OH}^{*}$

Nickel Cathode/Stainless Steel Anode, Exp. 25b and 25c

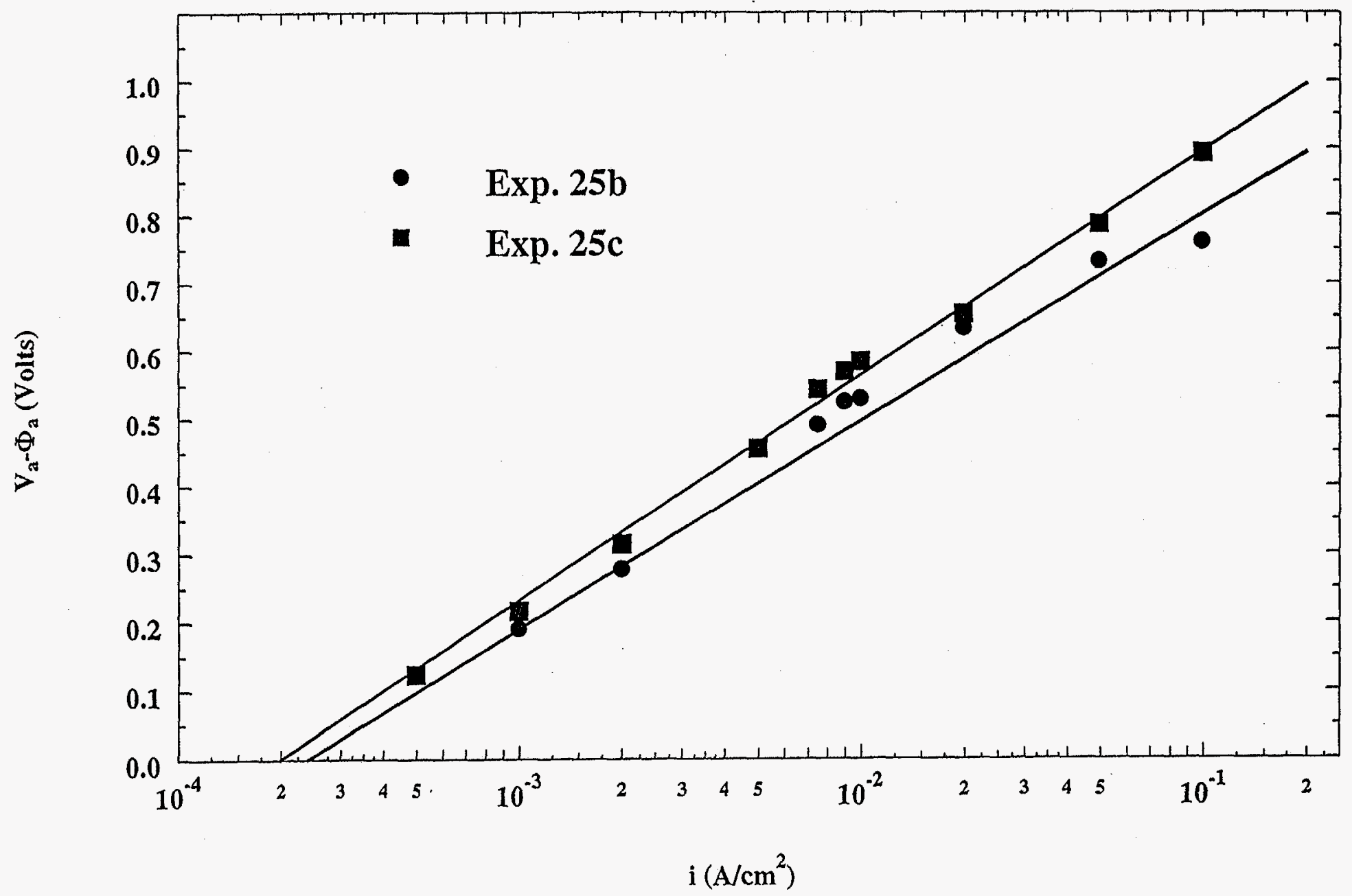




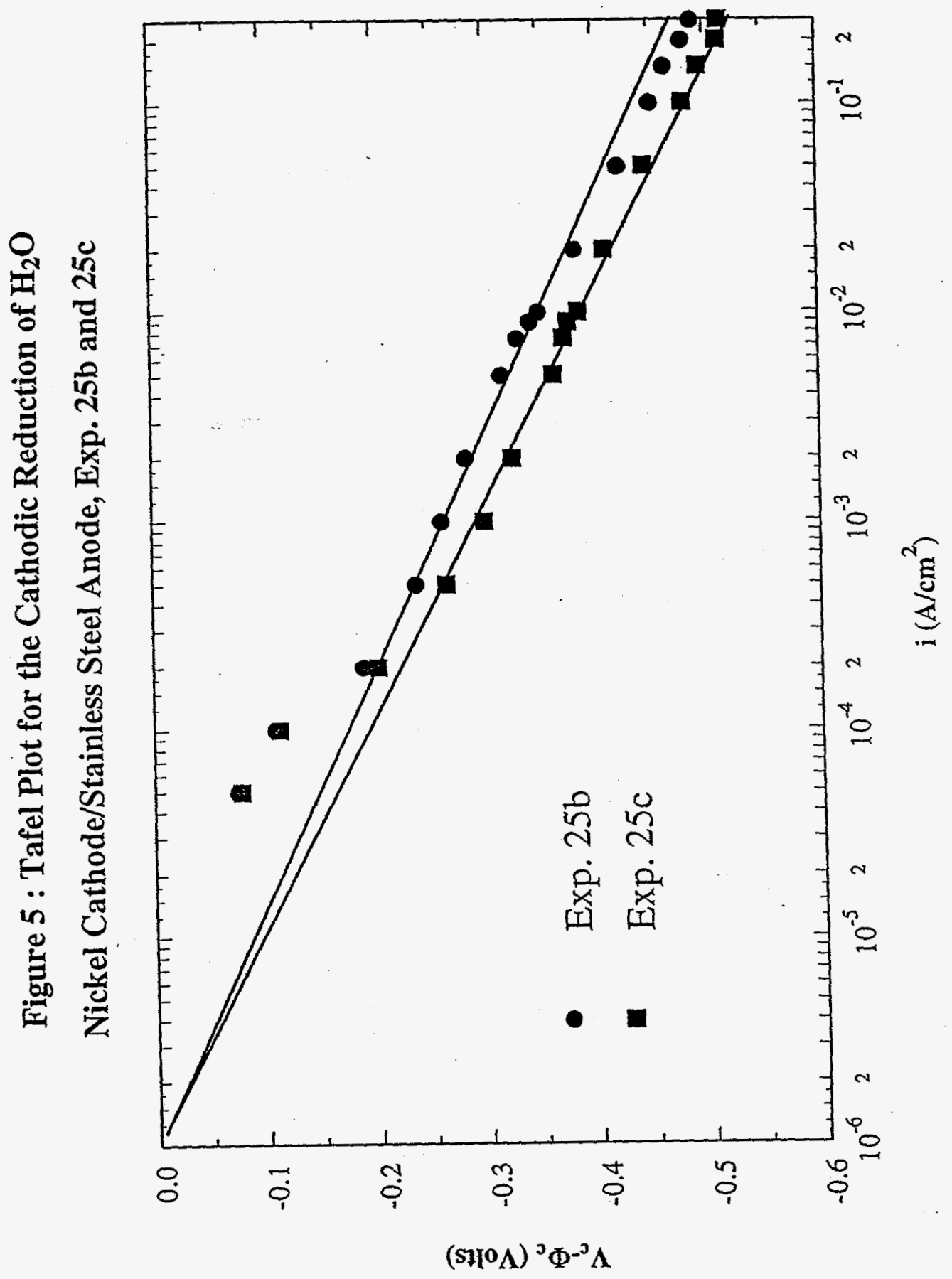




\subsection{Introduction}

\subsection{Model}

In section 2 of this report, experimental data were presented for the electrochemical reduction of $\mathrm{NaNO}_{3}, \mathrm{NaNO}_{2}$, and $\mathrm{NaOH}$ aqueous solutions. The purpose of this section is to present the numerical model that was used to evaluate the experimental results. Then in Section 4, the mathematical model is used with a multivariate nonlinear objective function and the data from Section 2 to estimate the exchange current densities and transfer coefficients for the reduction of $\mathrm{NaNO}_{3}$ and $\mathrm{NaNO}_{2}$ in alkaline solutions. The model is necessary because five competing reactions occur at the cathode and because the batch experimental data indicate that. the mass transfer and kinetics are coupled for industrially significant current densities and concentrations.

The model presented here extends the boundary layer model developed by Prasad $e t$ al.[5] to include non isothermal operation and water flux across a membrane. Parallel plate electrochemical cell models have been reviewed by Coleman and White [4] and parallel-plate cells operated in batch mode have been reviewed by Wingard [15]. For the electrochemical treatment of $\mathrm{NO}_{3}{ }^{-} / \mathrm{NO}_{2}{ }^{-}$, Coleman and White [4] developed a model which can be used to predict the conversion per pass, radial potential, and concentration profiles throughout the anolyte and catholyte of a separated parallel plate cell. The model includes the effects of ionic migration and diffusion, ohmic and charge-transfer resistances, and multiple reactions and uses the one-step method of Mader and White [16]. Also, the model was used to predict the offgas compositions, based on Henry's law, and liquid phase compositions in the recirculation tanks during batch operation. However, the model is computationally intensive which limits its usefulness in optimization studies.

The model presented by Prasad et al. [5] is a less computationally intensive model for the $\mathrm{NO}_{3}{ }^{-} / \mathrm{NO}_{2}^{-}$systems considered here. They used a boundary layer approximation at the electrode/solution interface and across the separator to account for diffusion and migration of ions. Comparison of the current versus potential predictions with Coleman's results [4] showed excellent agreement under similar operating conditions. Batch electrolysis conditions require the anolyte and catholyte to be recirculated through the cell and into mixing reservoirs. Typically, the residence time is short and the conversions per pass are small in the reactor due to the goal of operating at high limiting currents. This is a result of the kinetics and mass transfer limitations on the reactions. Under these conditions, it was shown that the boundary layer model [5] decreased the computation time by a factor of ten for $95 \% \mathrm{NO}_{3}{ }^{-} / \mathrm{NO}_{2}{ }^{-}$ destruction.

Also, the boundary layer model [5] has advantages over a laminar flow model such as that presented by Coleman and White [4] because of its versatility with respect to the flow characteristics and because it has minimal computation characteristics. A model was needed to describe the kinetic parameters and mass transfer characteristics in a separated parallel plate flow cell under laminar or turbulent operation. It was necessary that the model predict liquid phase bulk concentrations and off-gas flow rates for transient operation of the overall batch process. Furthermore, a model was needed that could be used to optimize and scale-up the process for possible pilot plant design. These aspects of the model broaden the scope of the literature review presented in this section to include contributions from boundary layer theory, 
parallel plate, and batch reactor models. These contributions are reviewed in sections 3.2.1 through 3.2.2.

\subsection{Literature Review for the Model}

\subsubsection{Boundary Layer and Algebraic Models}

The concept of a boundary layer and thus, mass transfer in the boundary layer was discussed in Bird et al. [17]. This simplified the analysis of transport processes in complex flow situations. Newman [18] presented a model to determine the effect of migration on limiting currents at a rotating disk, a growing mercury drop, a semi-infinite stagnant fluid, and a Nernst diffusion layer. This concept has since been expanded by Newman [19] to general electrochemical systems in which the effects of diffusion and migration are the dominant modes of transport within the boundary layer. Caban and Chapman [20] presented a model that used orthogonal collocation to solve ordinary differential equations for transport in the boundary layer. Current densities and concentration profiles were calculated which showed good agreement with rigorous calculations.

These models do not consider multiple reactions or a separated cell. A simple model was developed by Engelken and Van Doren [21] for the electrodeposition of CdTe and other II-VI or III-V compounds based upon a generalized Butler Volmer equation that considers ion transport limitations near the cathode surface. Lapicque and Storck [22] developed a model for the electrowinning of copper in a continuous parallel plate reactor in which diffusion and migration was taken into account in the boundary layer of each electrode. Edwards and Newman [23] developed a boundary layer model for a single reaction in an unseparated cell which did not include the effects of migration to show the interactions between diffusion in the boundary layer and the axial component of current.

Multiple reactions were considered by Simpson and White [24]. Their work presented a simple algebraic model for an isothermal, parallel plate, zinc/bromine flow cell in which the model used linear concentration and potential profiles in the boundary layers and the separator for a single reaction at each electrode. Smeltzer and Fedkiw $[25,26]$ presented boundary models for the reduction of nitrobenzene to $\mathrm{p}$-aminophenol at the cathode of a parallel plate reactor. Prasad et al. [5] developed an isothermal model to predict off-gas compositions and liquid phase compositions, in which they used a boundary layer approximation at the electrode/solution interface and across the separator to account for diffusion and migration of ions. The model presented in this dissertation extends that presented by Prasad et al. [5] to include non isothermal conditions.

\subsubsection{Batch Reactor Models}

Batch electrochemical models have been developed for a variety of processes because experimental evaluation of flow through cells with small conversions per pass and stirred-tank reactors can use this type of approach. Pickett [27] discussed a recirculating batch electrochemical reactor in which he noted that there were no existing theoretical predictions for the transient concentrations in terms of flow rate, electrode area, and electrolyte volumes. This work described a preliminary analysis for the electrowinning of copper using a parallel- 
plate electrochemical cell. Walker and Wragg [17] presented an approximate (i.e., steady state) and rigorous (i.e., transient) model for the prediction of concentration and current versus time relationships in electrochemical reactors with continuous recirculation and a well-mixed tank. The model was applied to a fluidized bed reactor for the cathodic removal of copper. Both of these models do not consider Butler-Volmer kinetics.

Scott [29-32] developed a batch electrochemical reactor model which considered independent and dependent parallel reactions as well as a parallel-series reaction scheme. The model showed reasonable agreement with experimental data for the oxidation of aqueous oxalic acid and glyoxylic acid solutions. In contrast to the models in this project, these models used Tafel kinetic expressions. Complete kinetic expressions were used by Nguyen $e t$ al. [33]. They presented a mathematical model for an electrochemical reactor with a continuous, stirred-tank reactor under total and partial recycles which was applied to the electrowinning of copper from aqueous hydrochloric acid. Weise $e t$ al. [34] presented a model for the electrochemical reduction of $\mathrm{N}$-nitroso-2-methylindoline to $\mathrm{N}$-amino-2-methyllindoline utilizing a batch stirred-tank electrochemical reactor. In this work, the influence of initial concentration, temperature, electrode area, and the hydrodynamics of the electrolyte on selectivity was evaluated.

Simpson and White [35] extended their algebraic model of a zinc/bromine flow cell to predict cell performance under batch operation. The model indicated that the charging operation of a zinc/bromine battery could be significantly improved by using a storage tank with a larger residence time for the bromine electrolyte. Bakshi and Fedkiw [36] developed a model that used optimal-control theory to improve selectivity in a well-mixed electrochemical batch reactor. Scott and Payton [37] presented a model for a batch recycle operation which describes the process of metal deposition from a mixed metal ion solution. In this work, the performance of the electrodeposition of heavy metal at low concentrations in a solution in the presence of dissolved iron is illustrated.

Coleman and White [4] developed a model that was used to predict the off-gas compositions, based on Henry's law, and liquid phase compositions in recirculation tanks during batch operation for the electrochemical destruction of $\mathrm{NO}_{3}{ }^{-} / \mathrm{NO}_{2}{ }^{-}$. However, the model is computationally intensive which limits its usefulness in optimization studies. The model of Prasad et al. [5] considered batch electrolysis conditions, for the reduction of $\mathrm{NO}_{3}^{-} / \mathrm{NO}_{2}^{-}$, which required the anolyte and catholyte to be recirculated through the cell and into mixing reservoirs.

\subsection{Model Equations}

In the model developed here, the well-mixed reservoirs of the anolyte and the catholyte side of the parallel plate cell are coupled through the separator with the well-mixed reservoir of the catholyte side of the cell. Each of these can be treated as batch reactors with gas generation. The electrolyte processes considered are the reduction of $\mathrm{NO}_{3}^{-}, \mathrm{NO}_{2}^{-}$, and $\mathrm{H}_{2} \mathrm{O}$ in the catholyte and the oxidation of $\mathrm{OH}^{-}$in the anolyte. Off-gas generation consists of $\mathrm{N}_{2}, \mathrm{~N}_{2} \mathrm{O}$, and $\mathrm{H}_{2}$ from the catholyte as well as $\mathrm{O}_{2}$ from the anolyte. The $\mathrm{NH}_{3}$ produced by the reduction of $\mathrm{NO}_{2}^{-}$was found both experimentally and through theoretical predictions to remain in solution at the dilute concentrations and the low temperatures used in the experiments. 
Material and energy balances are solved to predict concentration changes due to electrochemical kinetics, and potential and temperature changes due to heat transfer and ohmic losses within the electrochemical cell. Off-gas compositions and molar flow rates are predicted for transient operation by the model.

\subsubsection{Model Assumptions}

The model equations are developed by considering the following assumptions:

1. Only the oxidation of $\mathrm{OH}^{-}$occurs at the anode.

2. The only homogeneous reaction is ammonia hydrolysis.

3. Butler-Volmer kinetics apply and the reaction orders $\mathrm{p}_{\mathrm{kj}}$ and $\mathrm{q}_{\mathrm{kj}}$ follow stoichiometry.

4. The anolyte and catholyte reservoirs are well mixed and may be treated as batch reactors with gas generation.

5. Current is passed through the membrane by $\mathrm{Na}^{+}$ions. These ions are transported across the membrane to satisfy electroneutrality [19] of the combined system:

$$
\sum_{k} z_{k} C_{k}=0
$$

where $\mathrm{C}_{k}$ is the concentration of the positive and negative ions. These $\mathrm{Na}^{+}$ions are hydrated and the flux of water across the membrane is related to the flux of $\mathrm{Na}^{+}$with a proportionality factor $(\lambda)$ dependent on current density and temperature:

$$
N_{W}=\lambda(i, T) N_{N a^{+}}
$$

6. The activities of the species are equal to their concentration.

7. The partial pressure of $\mathrm{O}_{2}$ in the anolyte is equal to $1 \mathrm{~atm}$ and the concentration of dissolved gases $\mathrm{N}_{2}, \mathrm{~N}_{2} \mathrm{O}$, and $\mathrm{H}_{2}$ in the catholyte are always equal to their saturation values at the reservoir temperature.

8. The solubility of $\mathrm{NH}_{3}$ in the catholyte is governed by the Henry's Law constant [25]:

$$
H_{k}(T, P)=\frac{y_{k} P}{x_{k}}
$$

and is a function only of temperature and pressure. In dilute solutions $\mathrm{NH}_{3}$ remains in the liquid phase as dissolved $\mathrm{NH}_{3}$ or hydrolyzed $\mathrm{NH}_{4}{ }^{+}$.

9. The catholyte does not contain dissolved $\mathrm{O}_{2}$ and only the five reactions (1-5) occur at the cathode.

10. The conductivity through the boundary layer is constant and approximated by the bulk solution conductivity.

11. The initial values of the ionic species diffusion coefficients are given from infinite dilution theory and changes in the diffusion coefficients may be approximated using an infinite dilution approximation [19]: 


$$
\frac{D_{k} \mu}{T}=\text { constant }
$$

\subsubsection{Current and Voltage Equations}

The voltage of the cell $\left(V_{t}\right)$ depends on the open circuit potential of the cell $\left(V_{0}\right)$, the resistances of the anolyte, catholyte, and separator $\left(R_{a}, R_{c}\right.$, and $\left.R_{s}\right)$, the current density through the cell (I), and the overpotentials at the anode and cathode $\left(\mathrm{V}_{\mathrm{a}}-\phi_{\mathrm{a}}\right.$ and $\left.\mathrm{V}_{\mathrm{c}}-\phi_{\mathrm{c}}\right)$ :

$$
V_{t}=V_{o}+i A R_{\text {cell }}+\left|V_{a}-\phi_{a}\right|+\left|V_{c}-\phi_{c}\right|
$$

The solution potential at the anode and cathode are $\phi_{\mathrm{a}}$ and $\phi_{\mathrm{c}}$ respectively. $\mathrm{V}_{\mathrm{a}}$ and $\mathrm{V}_{\mathrm{c}}$ are the potentials of the anodic and cathodic electrodes. The geometric electrode area, $\mathrm{A}$, is used and $I$ is the sum of the partial current densities in the anolyte and catholyte:

$$
i=\sum_{j}^{\text {anode }} i_{j}=-\sum_{j}^{\text {cathode }} i_{j}
$$

The open circuit potential of the cell $\left(\mathrm{V}_{\mathrm{o}}\right)$ is the difference between the thermodynamic potential of the anode $\left(\mathrm{U}_{\mathrm{a}}\right)$ and the mixed potential of the cathode $\left(\mathrm{E}_{\mathrm{c}}\right)$ :

$$
V_{o}=U_{a}-E_{c}
$$

The thermodynamic potential of the anodic reaction deviates from the standard potential, according to the Nernst equation [39], as a function of the concentration of the species in the anolyte and the temperature change during the batch operation:

$$
U_{a}=U_{a}^{\theta}-\frac{R T}{4 F} \ln \left(\frac{C_{\mathrm{H}_{2} \mathrm{O} p_{\mathrm{O}_{2}}}^{2}}{C_{\mathrm{OH}^{-}}^{4}}\right)
$$

The voltage of the cathode $\left(\mathrm{E}_{\mathrm{c}}\right)$ is a mixed potential corresponding to the combined effects of thermodynamics and kinetics of $\mathrm{NO}_{3}^{-} / \mathrm{NO}_{2}{ }^{-}$reduction reactions and the oxide films that may form on the surface.

The resistance in the cell depends on the conductivity of the anolyte, catholyte, and the solution in the separator $\left(\kappa_{s}\right)$. It is also directly dependent on the perpendicular distances $\left(S_{i}\right)$ through the electrolytes and separator between the parallel plates of the cell:

$$
R_{c e l l}=\frac{1}{A}\left(\frac{S_{a} N_{M, a}}{\kappa_{a}}+\frac{S_{s} N_{M}}{\kappa_{s}}+\frac{S_{c} N_{M, c}}{\kappa_{c}}\right)
$$

Where, the MacMullin number [40] of the separator $\left(\mathrm{N}_{\mathrm{M}}\right)$ and the MacMullin numbers of the 
turbulence promoters in the anolyte and catholyte $\left(\mathrm{N}_{\mathrm{M}, \mathrm{a}}\right.$ and $\left.\mathrm{N}_{\mathrm{M}, \mathrm{c}}\right)$ are a function of the porosity and tortuosity of the separator and turbulence promoters respectively. These MacMullin numbers (i.e., $\mathrm{N}_{\mathrm{M}}, \mathrm{N}_{\mathrm{M}, \mathrm{a}}$, and $\mathrm{N}_{\mathrm{M}, \mathrm{c}}$ ) were determined experimentally using impedance methods. The solution conductivity $(\kappa)$ was calculated using the empirical correlation shown in Appendix A.

The partial current densities and the overpotentials at the anode or cathode may be determined by using a concentration dependent form of the Butler-Volmer equation:

$$
\frac{i_{j}}{i_{o j, \text { ref }}}=\prod_{k}\left(\frac{C_{k}}{C_{k, \text { ref }}}\right)^{p_{k j}} \exp \left(\frac{\alpha_{a j} F}{R T} \eta_{j}\right)-\prod_{k}\left(\frac{C_{k}}{C_{k, \text { ref }}}\right)^{q_{k j}} \exp \left(\frac{-\alpha_{c j} F}{R T} \eta_{j}\right)
$$

where the reaction orders are:

and reactions (1-6) are written in a generalized form:

$$
\begin{aligned}
& p_{k j}=s_{k j} \text { and } q_{k j}=0 \text { if } s_{k j}>0 \\
& p_{k j}=0 \text { and } q_{k j}=-s_{k j} \text { if } s_{k j}<0
\end{aligned}
$$

$$
\begin{gathered}
\sum_{j} s_{k j} M_{k}^{z_{k}}-n_{j} e^{-} \text {where } n_{j}=1 \\
\eta_{j}=V_{c}-\phi_{c}-U_{j, \text { ref }} \text { for the cathodic reactions } \\
\eta_{j}=V_{a}-\phi_{a}-U_{j, \text { ref }} \text { for the anodic reactions }
\end{gathered}
$$

where $\mathrm{k}$ is the ionic species and $\mathrm{j}$ is the corresponding reaction. The overpotential $\left(\eta_{\mathrm{j}}\right)$, in equation (11), is the difference between the voltage of the electrode, the solution potential at the electrode surface, and the reference potential of the electrochemical reaction. where the thermodynamic potential of reaction $j$ relative to reference concentrations $\left(C_{k, \text { ref }}\right)$ and can be expressed:

$$
U_{j, r e f}=U_{j}^{\theta}-\frac{R T}{n_{j} F} \sum_{k} s_{k j} \ln \left(\frac{C_{k, r e f}}{a_{k}^{\theta}}\right)
$$

For multiple reactions, the concentration ratio, in equation (11), depends on the sum of the partial current densities since the flux of species $k$ at the surface depends on all the reactions. An expression for this ratio is obtained by using the flux expression at the electrode surface. The boundary layer approximation allows us to write the flux, $N_{k}$, as the sum of the effects of diffusion and migration: 


$$
N_{k}=-D_{k} \frac{d C_{k, b l}}{d x}+\frac{z_{k} D_{k} F i}{R T \kappa} C_{k, b l}
$$

where $C_{k, b l}$ is the concentration of species $k$ in the boundary layer and $\kappa$ is equal to $\kappa_{c}$ for the catholyte or $\mathrm{k}_{\mathrm{a}}$ for the anolyte boundary layers. Also, the flux of species $\mathrm{k}, \mathbf{N}_{\mathrm{k}}$ at the electrode is equal to the production or consumption of that species in each reaction:

$$
N_{k}=\sum_{j} \frac{-s_{k j} i_{j}}{n F}
$$

Since we assume the flux of species $k$ is consistent across the boundary layer, equation (16) may be placed in a first-order differential form:

$$
\frac{d C_{k, b l}}{d x}-\frac{\gamma_{k} C_{k, b l} k_{m k}}{D_{k}}=-\frac{N_{k}}{D_{k}}
$$

where $\gamma_{\mathbf{k}}$ is the contribution to equation (18) by bulk migration:

$$
\gamma_{k}=\frac{z_{k} F D_{k} i}{R T \kappa k_{m k}}
$$

Integrating between the following boundary conditions:

$$
\begin{aligned}
& @ \mathrm{x}=0, \mathrm{C}_{\mathrm{k}, \mathrm{b}}=\mathrm{C}_{\mathrm{kb}} \\
& @ \mathrm{x}=\delta=\mathrm{D}_{\mathrm{k}} / \mathrm{k}_{\mathrm{mk}}, \mathrm{C}_{\mathrm{k}, \mathrm{bl}}=\mathrm{C}_{\mathrm{k}}
\end{aligned}
$$

where $\delta$ is the boundary layer thickness. This gives an expression for the ratio of the surface concentration, $\mathrm{C}_{\mathrm{k}}$, to the bulk concentration $\left(\mathrm{C}_{\mathrm{kb}}\right)$ :

$$
\frac{C_{k}}{C_{k b}}=\left(\frac{N_{k}}{C_{k b} \gamma_{k} k_{m k}}\right)+\left(1-\frac{N_{k}}{C_{k b} \gamma_{k} k_{m k}}\right) e^{\gamma_{k}}
$$

Therefore, the concentration ratio in equation (11) may be derived from equation (36):

$$
\frac{C_{k}}{C_{k, \text { ref }}}=\left(\frac{N_{k}}{C_{k b} \gamma_{k} k_{m k}}\right)+\left(1-\frac{N_{k}}{C_{k b} \gamma_{k} k_{m k}}\right) e^{\gamma_{k}} \frac{C_{k b}}{C_{k, \text { ref }}}
$$

and $\mathrm{k}_{\mathrm{mk}}$ is the mass transfer coefficient calculated by the following equation:

$$
k_{m k}=\frac{N u_{k} D_{k}}{d_{e}}
$$

where $d_{e}$ is the hydraulic diameter of the electrochemical cell which for long electrodes: 


$$
d_{e}=\frac{2 S L}{(S+L)} \Rightarrow 2 S \text { where } L>S
$$

\subsubsection{Reservoir Mass and Energy Balances}

Once the partial current densities are determined, the liquid phase concentrations and molar off-gas flow rates are calculated. The mass balance equations are written, for the batch reactors of the anolyte and catholyte, assuming single pass conversion is low. The transient changes in the reservoir concentrations depend on the partial current densities and the volume change in the reactor. For a general mass balance:

$$
\frac{d\left(V C_{k}\right)}{d t}=Q_{\text {in }} C_{k, \text { in }}-Q_{\text {out }} C_{k, \text { out }}-\sum_{j} \frac{s_{k j} i_{j} A}{n_{j} F}+R_{k}
$$

The $\mathrm{Q}_{\mathrm{in}}$ and $\mathrm{Q}_{\text {out }}$ terms allow for additions of electrolyte or sample of the reservoir volumes. Under batch operation with negligible homogeneous reactions, this can be written for the liquid phase species $\mathrm{NO}_{3}^{-}, \mathrm{NO}_{2}^{-}$, and $\mathrm{OH}^{-}$of the anolyte and catholyte:

$$
\frac{d\left(V C_{k b}\right)}{d t}=-\sum_{j} \frac{s_{k j} i_{j} A}{n_{j} F}
$$

and (according to assumption 5) for $\mathrm{Na}^{+}$:

$$
-\left(\frac{d V C_{\mathrm{Na}^{+}}}{d t}\right)_{\text {catholyte }}=\left(\frac{d V C_{\mathrm{Na}^{+}}}{d t}\right)_{\text {anolyte }}=-\frac{i \mathrm{~A}}{4 F}
$$

The energy balance has contributions from the kinetics and thermodynamics of the electrochemical reactions, resistive potential losses, and the energy associated with the centrifugal pumping action which all serve to increase the temperature of the process. Ambient heat transfer and the off-gas production remove energy from the process and thus, act to decrease the temperature. For an electrochemical batch reactor with gas generation the energy balance can be written:

$$
\begin{array}{r}
V \rho C_{p} \frac{d T}{d t}=-\left(\left|V_{a}-\phi_{a}\right| i+\sum_{j}\left|V_{c j}-\phi_{c j}\right| i_{c j}\right) A \\
-(i A)^{2} R_{c e l l}+U A\left(T_{A i r}-T\right) \\
-Q C_{p g a s} T+H_{p}
\end{array}
$$

The first term on the right-hand side corresponds to the summation of the energy losses at the anode and cathode due to kinetics and mass transfer. The second term corresponds to internal resistance losses due to the anolyte, catholyte, and separator. The third and fourth terms are the energy loss due to heat transfer to the atmosphere and the off-gas leaving the reservoirs, 
respectively. The fifth term $\left(\mathrm{H}_{\mathrm{p}}\right)$ is the energy input into the process due to the centrifugal pumps. This term may be evaluated in terms of the hydraulic efficiency $\left(\eta_{p}\right)$ [41] of the centrifugal pumps:

$$
H_{p}=E_{p}\left(1-\eta_{p}\right)
$$

in which $E_{p}$ is the energy output or horsepower rating of the centrifugal pumps.

\subsubsection{Membrane Flux Analysis}

The volumes of the anolyte and catholyte change during the course of an experimental run due to hydration of the $\mathrm{Na}^{+}$which is transported across the separator. Typically, $\mathrm{Na}^{+}$ions are hydrated with 3 to 4 molecules of water when a 350 Nafion membrane is used in a low current density chlor/alkali process [42]. In the reduction of $\mathrm{NO}_{3}{ }^{-} / \mathrm{NO}_{2}{ }^{-}$, high current densities are used and the number of molecules of water transported with each $\mathrm{Na}^{+}$ion is dependent on temperature and electroosmosis. This electroosmotic affects increases with increasing current density. A ratio of the number of molecules of water transported with each $\mathrm{Na}^{+}$ion $(\lambda(\mathrm{i}, \mathrm{T})$ ) may be defined and used to account for the volume change:

$$
\frac{d V}{d t}=-\frac{i}{4 F} \frac{A M_{w}}{\rho} \lambda
$$

in which $\mathbf{M}_{\mathrm{w}}$ and $\rho$ are the molecular weight and density of water respectively. This ratio $(\lambda)$ will be evaluated in section 5.3 (Model Predictions) utilizing the numerical model predictions in comparison to experimental data. The volume change affects the transient concentrations of the liquid species in the anolyte and catholyte. Thus, the kinetic rates and partial current densities of the cathodic reactions are affected by the amount of water transported across the membrane.

\subsubsection{Off-Gas Generation}

In full simulant batch experiments, the analysis of the off-gas generation from the catholyte is complicated by the non steady state nature of the start-up and a changing gas volume above the catholyte throughout the experiment. A mass balance was performed to model the gas fractions in the off-gas:

$$
\frac{R T}{P} \frac{d N_{g a y} y_{l}}{d t}=F_{i n} y_{l, \text { in }}-F_{\text {out }} y_{l, o u t}+\sum_{j} \frac{s_{l j} i_{j} A}{n_{j} F} \frac{R T}{P}
$$

where $\mathrm{N}_{\mathrm{gas}}$ is the total moles of gas in the reservoir, and $\mathrm{y}_{1}$ is the mole fraction of gas species 1 . Now, since the catholyte reservoir, piping and side of the parallel pore plate have a fixed total volume: 


$$
\frac{d V_{\text {total }, c}}{d t}=\frac{d V_{c, \text { liq }}}{d t}+\frac{d V_{c, g a s}}{d t}=0
$$

Thus, at normal pressures of operation, the gas phase is ideal

$$
y_{l} \frac{d N_{g a s}}{d t}=-y_{l} \frac{P}{R T} \frac{d V_{c, l i q}}{d t}
$$

A total mole balance on the gas in the reservoir can be written:

$$
\frac{d N_{\text {gas }}}{d t}=\frac{F_{\text {in }} P}{R t}-\frac{F_{\text {out }} P}{R T}+\sum_{l} \sum_{j} \frac{s_{l j} i_{j} A}{n F}
$$

where the sum over $l$ accounts for gas species only and $\mathrm{j}$ sums over all reactions. This can be rearranged to solve for the outlet gas flow:

$$
F_{\text {out }} \frac{P}{R T}=\frac{P}{R T} \frac{d V_{c, l i q}}{d t}+F_{\text {in }} \frac{P}{R T}+\sum_{l} \sum_{j} \frac{s_{l j} i_{j} A}{n_{j} F}
$$

A simplified equation for the mole fraction change in the reservoir can be obtained using the chain rule on equation (32) and multiplying equation (35) by $y_{1}$ and subtracting the resultant equations to give:

$$
N_{\text {gas }} \frac{d y_{l}}{d t}=-y_{l} \frac{F_{i n} P}{R T}-y_{l} \sum_{l} \sum_{j} \frac{s_{l j} i_{j} A}{n_{j} F}+\sum_{j} \frac{s_{l j} i_{j} A}{n_{j} F}
$$

where we assumed $y_{\text {lim }}=0$. The sum is over the reactions (3-5) which produce off-gas and $F_{i n}$ is the inlet flow rate of argon which is constant at $8.3 \times 10^{-5}$ liters/sec. Equation (34) may be integrated to give an expression for the total moles of gas in the reservoir above the liquid catholyte:

$$
N_{\text {gas }}=\frac{P\left(V_{\text {total }}-V_{c, \text { liq }}\right)}{R T}
$$

where $V_{\text {total }}$ is the total volume of the reservoir. It should be noted that $V_{c \text {,liq }}$ must be determined by numerical integration in the Runge-Kutta routine as a function of time. The instantaneous species off-gas flow rate (moles/sec) out of the catholyte reservoir may be calculated by the equation:

$$
y_{l} F_{\text {out }} \frac{P}{R T}=y_{l}\left(\frac{P}{R T} \frac{d V_{c, l i q}}{d t}+F_{i n} \frac{P}{R T}+\sum_{l} \sum_{j} \frac{s_{i j} i_{j} A}{n F}\right)
$$


$\mathrm{F}_{\text {out }}$ and $\left(\mathrm{dV}_{\mathrm{c}, \mathrm{iq}} / \mathrm{dt}\right)$ has units of liters/sec. These equations (35-37) allowed the prediction of the molar flow rate of the off-gas species and the mole fraction of these species in the off-gas.

\subsubsection{Solution Technique and Model Algorithm}

The material balances for the anolyte and catholyte volume, the concentration of $\mathrm{NO}_{3}{ }^{-}$ and $\mathrm{NO}_{2}{ }^{-}$in the catholyte, and the concentration of $\mathrm{OH}^{-}$and $\mathrm{Na}^{+}$in both the catholyte and anolyte and the energy balance for the system temperature $(T)$ were integrated with respect to time using a fourth order Runge-Kutta routine with a variable step size dependent upon the potential change. At each time step, six equations corresponding to the five cathodic reactions (1-5) described by equation (11) and the sum in equation (6) were solved simultaneously using a modified Newton-Raphson technique [44] for the partial current densities of reactions (1-5) and the cathodic potential. These partial current densities and thus, the liquid phase ionic compositions of the catholyte and the off-gas flow rates are dependent upon the exchange current densities and transfer coefficients used in the calculations. Other factors which affect these calculations include the physical properties of the electrolytes, mass transfer of the ionic species within the boundary layer, and the thermodynamics of the ionic species.

An outline of how the model equations were used in the routine and the sequence of their use is shown in Figure 6. At time equal to zero, the partial current densities and cathodic potential are calculated from the initial conditions of concentration, volume, and temperature in the Newton-Raphson IMSL subroutine. The anodic potential and current efficiencies are calculated prior to adjusting the anolyte and catholyte volumes and concentrations for sampling. These results are then printed prior to integration of the concentrations, volumes, temperatures, and off-gas mole fractions over time in the Runge-Kutta routine. The instantaneous off-gas flow rates are then calculated. The convergence criteria is $95 \%$ destruction of the sum of the $\mathrm{NO}_{3}^{-}$and $\mathrm{NO}_{2}^{-}$concentrations. If this is not satisfied, the physical properties and mass transfer parameters of the electrolytes are recalculated at the time step. The partial cathodic current densities, anodic and cathodic potentials, and the current efficiencies are then recalculated prior to printing.

\subsection{Introduction}

\section{Parameter Estimation}

A model and an objective function are required to analyze the data of Section 2 because five competing reactions occur at the cathode and because the batch experimental data reported in Section 2 indicate that the mass transfer and kinetics are coupled for industrially significant current densities and concentrations. Parameters for one of the five reactions, $\mathrm{H}_{2} \mathrm{O}$ reduction, were determined in Section 2 of this report. Here, the exchange current densities and transfer coefficients for the four competing cathodic reactions, involving the reduction of $\mathrm{NO}_{3}^{-}$and $\mathrm{NO}_{2}^{-}$, are determined. The parameters with their $95 \%$ confidence intervals indicate that nitrogen and ammonia are the predominant products. Nitrous oxide is produced in small amounts below $0.25 \mathrm{~A} / \mathrm{cm}^{2}$. Estimates, consistent with these kinetic parameters, of the moles of $\mathrm{H}_{2} \mathrm{O}$ transported per mole of $\mathrm{Na}^{+}(\lambda)$ transported through the Nafion ${ }^{\otimes}$ membrane from the anolyte to the catholyte in the parallel plate electrolyzer are in the range of 8 and 10 and they depend on the temperature and the current density of the process. 
Figure $6:$ Flow Diagram for Numerical Model (Nitrate/Nitrite Reduction)

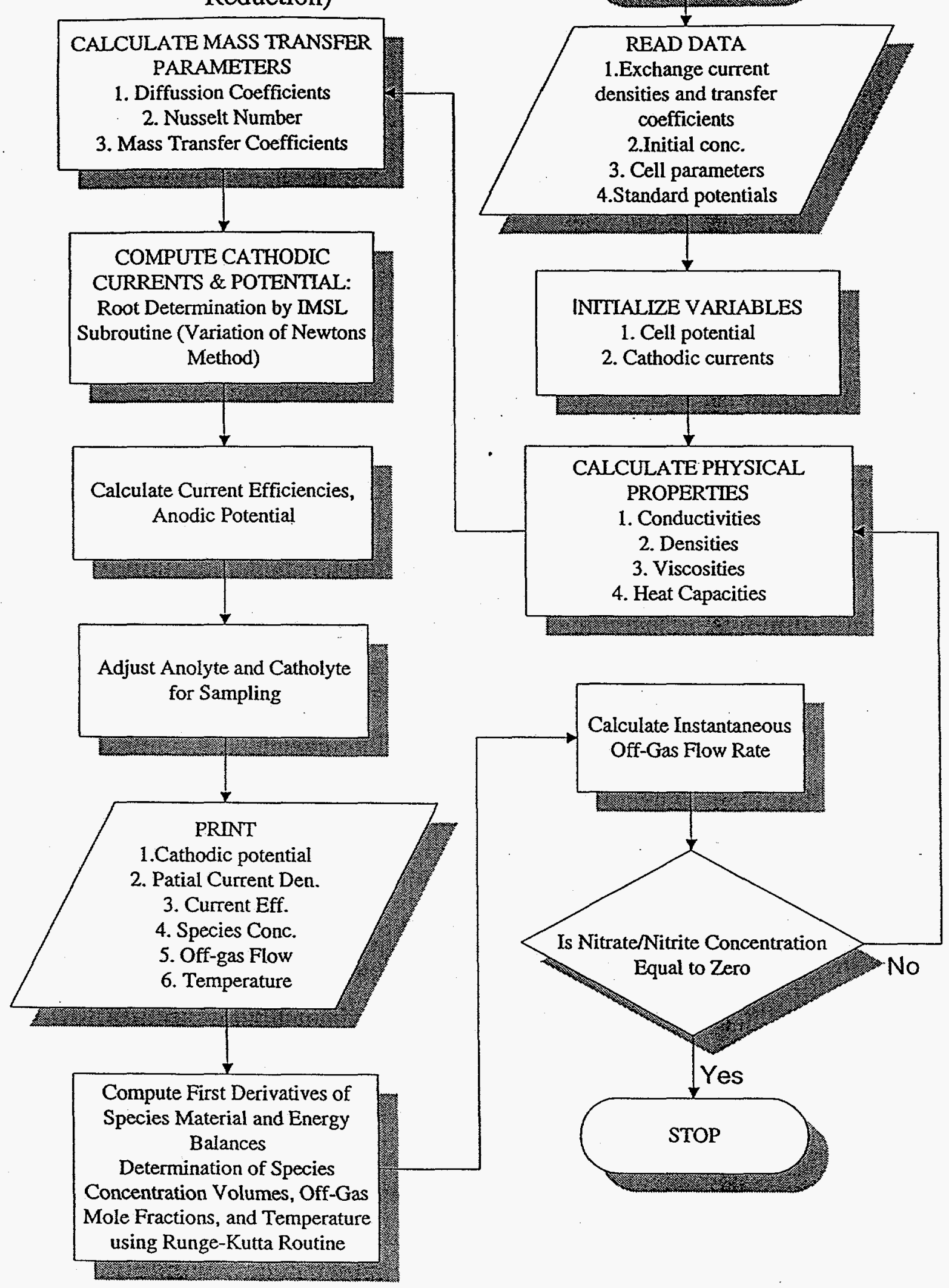




\subsection{Objective functions}

A systematic approach is needed to evaluate the experimental data from the $2^{3}$ factorial design and thereby determine the kinetic parameters for reactions (2-4). Tables 1A and 1B of Section 2 show the experimental design and the run numbers of the data used for the parameter estimation. The exchange current densities and the transfer coefficients of reactions (2-4) were determined by minimizing a nonlinear least squares objective function. In this function, the predicted dependent variables were determined from the numerical model and compared to the experimental dependent variables:

$$
\begin{aligned}
& f\left(C_{N H_{3}}, F_{N_{2}}, F_{N_{2} O}, \phi_{c}\right)=\frac{\sum_{l=0}^{N}\left(C_{N H_{3}, l}^{o b s}-C_{N H_{3}, l^{2}}^{\text {pred }}\right)^{2}}{\sigma_{F_{N H_{3}, l}}^{2}}+\frac{\sum_{l=0}^{N}\left(F_{N_{2}, l}^{o b s}-F_{N_{2}, l}^{\text {pred }}\right)^{2}}{\sigma_{F_{N_{2},}}^{2}} \\
& +\frac{\sum_{l=0}^{N}\left(F_{N_{2} O, l}^{o b s}-F_{N_{2} O, l}^{\text {pred }}\right)^{2}}{\sigma_{F_{N_{2} O, l}}^{2}}+\frac{\sum_{l=0}^{N}\left(\phi_{c_{l}}^{\text {obs }}-\phi_{c_{l}}^{\text {pred }}\right)^{2}}{\sigma_{\phi_{c_{l}}}^{2}}
\end{aligned}
$$

the four terms on the right-hand side of equation 38 correspond to the four dependent variables: the concentration of $\mathrm{NH}_{3}$ in the catholyte, the molar flow rates of the nitrogen and nitrous oxide in the off-gas, and the cathodic potential.

The exchange current densities and transfer coefficients for reactions (2-4) which minimize the function in equation (38) might be found using a nonlinear optimization program which combines a program for the model equations and a standard minimization subroutine such as might be found in the IMSL package. However, a high degree of sensitivity of the model convergence equations was observed with respect to the initial guesses of the partial current densities and the cathodic potential as discussed above. Thus, a less automatic step-wise methodology was used based on calculating the objective function for a fixed set of estimates of exchange current densities and transfer coefficients. That is, the minimum in equation (38) was searched by using designed sets of $2^{3}$ factorial designs. First, the transfer coefficients (around previous estimates of these kinetic parameters which are referred to as median values) were fixed while looking for changes in the value of the objective function at high and low values of $i_{0,2}, i_{0,3}$, and $i_{0,4}$. A path to the minimum was determined by the steepest decent caused by changes in the parameters. Then at the minimum, the exchange current densities were fixed while looking for changes in the value of the objective function at high and low values of $\alpha_{2}, \alpha_{3}$, and $\alpha_{4}$. Again, a path to the minimum was chosen by considering the steepest decent cause by changes in these parameters. This alternating procedure was repeated for physically meaningful values of all six parameters.

The magnitude of the step size during the search was varied (e.g., $0.01,0.1$, or 0.2 for the transfer coefficient and 1 or 2 orders of magnitude for the exchange current density) depending on the degree of accuracy required. It is important to note that the model predictions are correlated and not statistically independent because of the time-stepping. This is an inherent problem, albeit 
an insignificant one for these data, in the analysis of batch reactor data. In the evaluation of each set of parameters, a new set was accepted if they produced a lower objective function sum in which the degree of statistical certainty was $95 \%$ or greater. This was determined using ANOVA (analysis of variance) techniques in which the statistical $F$ and $t$ tests were applied over the sample (see Ref 15).

The magnitude of the interactions between the dependent and independent variables were considered in these designs. For example, the catholyte concentration of $\mathrm{NO}_{2}^{-}$and thus, $\mathrm{NH}_{3}$, is a strong function of the cathodic exchange current densities $\left(i_{j}\right)$ and the moles of water transported across the membrane per mole of $\mathrm{Na}^{+}(\lambda)$. The transient potential and temperature are a strong function of the cathodic transfer coefficients, the MacMullin number $\left(\mathrm{N}_{\mathrm{M}}\right)$, the overall heat transfer coefficient $(U)$ and relatively insensitive to the cathodic exchange current densities. Other factors must be considered in the comparison of the model predictions to experimental data using the nonlinear least squares objective functions (i.e., equations (38) and (39)).

The catholyte concentration of $\mathrm{OH}^{-}$is a strong function of the moles of water transported across the membrane per mole of $\mathrm{Na}^{+}(\lambda)$ and relatively insensitive to the cathodic exchange current densities because each of reactions (1-5) produces an approximately equivalent amount of $\mathrm{OH}^{-}$(i.e., 1 to 1.5 moles $\mathrm{OH}^{-}$per mole of electrons) per coulomb of charge passed. The transient potential and temperature are a strong function of the MacMullin number $\left(\mathrm{N}_{\mathrm{M}}\right)$ and the overall heat transfer coefficient $(U)$, respectively. Also, the value of the anodic exchange current density $\left(i_{o a}\right)$ and transfer coefficient $\left(\alpha_{\mathrm{a}}\right)$ were calculated by a Tafel analysis of experimental currentpotential scan data for the oxidation of $\mathrm{OH}^{-}$in the anolyte. These values, $i_{o a}$ and $\alpha_{a}$, are used to calculate the anodic potential which directly affects the predictions of the total cell potential.

Transient temperature changes in the electrochemical process affect the rates of reaction, due to the dependency of the diffusion coefficients, reference potentials, MacMullin number, physical properties and conductivities on temperature. In a non isothermal process in which the temperature increases, this results in higher mass transfer rates of the ionic species (i.e., $\mathrm{NO}_{3}^{-}$and $\mathrm{NO}_{2}{ }^{-}$at the cathode) in the boundary layer, thus increasing the limiting current density for the reduction of these species. Total cell voltage and concentration changes in the ionic species were also directly and indirectly dependent on temperature changes

For the determination of the exchange current density and transfer coefficient of reaction (1), a similar procedure was used. Tables $1 \mathrm{~A}$ and $1 \mathrm{~B}$ show the experimental design and the three independent variables; (1) the concentration of $\mathrm{NO}_{3}^{-},(2)$ temperature (T) and (3) current density. The dependent variables are the concentration of $\mathrm{NO}_{2}^{-}$and the cathodic potential. The off-gas and liquid phase reduction products of $\mathrm{NO}_{2}{ }^{-}$in the experiments of type 3 were too small to quantify by $\mathrm{GC}$ analysis of the off-gas and Kjeldahl analysis for $\mathrm{NH}_{3}$ in the catholyte and, therefore, these quantities were not used as dependent variables in the experimental design. An objective function, similar expression to equation (38), was developed for the reduction of $\mathrm{NO}_{3}{ }^{-}$:

$$
f\left(C_{N O_{2}^{-}}, \phi_{c}\right)=\frac{\sum_{l=0}^{N}\left(C_{N O_{2}^{-}, l}^{\text {obs }}-C_{N O_{2}^{-}, l}^{\text {pred }}\right)^{2}}{\sigma_{F_{N O_{2}^{-}, l}}^{2}}+\frac{\sum_{l=0}^{N}\left(\phi_{c_{l}}^{\text {obs }}-\phi_{c_{l}}^{\text {pred }}\right)^{2}}{\sigma_{\phi_{c_{l}}}^{2}}
$$


This objective function was minimized by searching the parameter space for the exchange current density $\left(i_{o 1}\right)$ and transfer coefficient $\left(\alpha_{1}\right)$ in the constant current density experiments outlined above. However, in this case these kinetic parameters were varied in a $3^{2}$ factorial manner around previous estimates during the search.

Empirical correlations for the physical properties of electrolyte solutions composed of $\mathrm{NaNO}_{3}, \mathrm{NaNO}_{2}$, and $\mathrm{NaOH}$ at temperatures of $30,45,60$, and $85^{\circ} \mathrm{C}$ were developed and used in the numerical model. Physical property expressions were developed for the density, viscosity, conductivity, and heat capacity of these electrolyte solutions as a function of concentration and temperature as shown in Appendix A. These expressions were an integral part of the numerical model in the calculations of the mass transfer parameters, kinetic expressions, material and energy balances in which the calculations were dependent on these physical properties. An outline of the methods used to calculated thermodynamic, mass transfer, and kinetic parameters are shown in Table 7. 
Table 7. Outline of the Procedure to Calculate Thermodynamic, Mass Transfer, and Kinetic Parameters.

\begin{tabular}{|c|c|c|}
\hline Model Parameters & Independent Variables & Procedure \\
\hline MacMullin number $\left(\mathrm{N}_{\mathrm{M}}\right)$ & $\begin{array}{c}\text { empirical correlation: } \\
\mathrm{N}_{\mathrm{M}}=72.7-(1.35) \mathrm{T}\left({ }^{\circ} \mathrm{C}\right) \text { for } \\
\mathrm{Nafion} 350 \text { membrane, } \mathrm{N}_{\mathrm{M}, \mathrm{a}}= \\
\mathrm{N}_{\mathrm{M}, \mathrm{c}}=2.40 \text { for the turbulence } \\
\text { promoters }\end{array}$ & $\begin{array}{c}\text { was experimentally determined } \\
\text { as a function of temperature } \\
\text { using } \mathrm{AC} \text { Impedance }\end{array}$ \\
\hline Diffusion Coefficients $\left(D_{k}\right)$ & temperature $(T)$, viscosity $(\mu)$ & $\begin{array}{l}\text { calculated using infinite dilution } \\
\text { diffusion coefficients adjusted for } \\
\text { temperature and viscosity } \\
\text { variations }\end{array}$ \\
\hline Mass Transfer Coefficients $\left(\mathrm{k}_{\mathrm{ml}}\right)$ & $\begin{array}{l}\text { Reynolds number(Re), Schmidt } \\
\text { number }\left(\operatorname{Sc}_{\mathrm{k}}\right) \text {; physical properties } \\
\text { - density }(p) \text {, viscosity }(\mu)\end{array}$ & $\begin{array}{l}\text { calculated using equation (11); a } \\
\begin{array}{c}=0.98, b=0.62 \text { for an MP cell } \\
\text { and } a=0.74, b=0.62 \text { for an FM- } \\
01 \text { cell }\end{array}\end{array}$ \\
\hline Reference Potentials(U) & $\begin{array}{l}\text { temperature }(\mathrm{T}), \text { bulk } \\
\text { concentration }\left(\mathrm{C}_{\mathrm{kb}}\right)\end{array}$ & $\begin{array}{l}\text { calculated using the Nernst } \\
\text { equation (8) }\end{array}$ \\
\hline $\begin{array}{c}\text { Exchange Current } \\
\text { Densities }\left(i_{05,}, i_{o 6}\right) \text { and Transfer } \\
\text { Coefficients }\left(\alpha_{o 5}, \alpha_{o 6}\right) \text { for } \\
\text { Reactions }(5) \text { and }(6)\end{array}$ & $\begin{array}{c}\text { temperature }(T) \text {, current } \\
\text { density }(\mathrm{T}) \text {, bulk } \\
\text { concentrations }\left(\mathrm{C}_{\mathrm{kb}}\right) \text {, cathode and } \\
\text { anode materials }\end{array}$ & $\begin{array}{c}\text { determined by Tafel analysis in } \\
\text { which the cell current density is } \\
\text { varied from } 1.0 \times 10^{-4} \text { to } 0.25 \\
\mathrm{~A}_{\mathrm{cm}} \mathrm{cm}^{2} \text { and } \mathrm{V}_{\mathrm{c}}-\phi_{\mathrm{c}} \text { is measured } \\
\text { with an anolyte and catholyte } \\
\text { composed of approximately } 3 \mathrm{M} \\
\mathrm{NaOH}\end{array}$ \\
\hline $\begin{array}{l}\text { Exchange Current Density }\left(i_{o l}\right) \\
\text { and Transfer Coefficient }\left(\alpha_{o 1}\right) \text { for } \\
\text { Reaction (1) }\end{array}$ & $\begin{array}{l}\text { temperature( }(T), \text { cell current } \\
\text { density }(\mathrm{I}), \text { bulk } \\
\text { concentrations }\left(\mathrm{C}_{\mathrm{kb}}\right) \text {, cathode } \\
\text { material }\end{array}$ & $\begin{array}{l}\text { determined by minimization of } \\
\text { the objective function ( } 38 \text { ) using } \\
\text { the numerical migration model } \\
\text { and the experiments }\end{array}$ \\
\hline $\begin{array}{c}\text { Exchange Current } \\
\text { Densities }\left(i_{\circ 2,}, i_{\circ 3}, i_{\circ 4}\right) \text { and Transfer } \\
\text { Coefficients }\left(\alpha_{2}, \alpha_{3}, \alpha_{4}\right)\end{array}$ & $\begin{array}{l}\text { temperature }(T) \text {, cell current } \\
\text { density }(\mathrm{I}) \text {, cathode material }\end{array}$ & $\begin{array}{l}\text { determined by minimization of } \\
\text { the objective function (13) using } \\
\text { a } 2^{3} \text { factorial design in the } \\
\text { numerical migration model and } \\
\text { the experiments }\end{array}$ \\
\hline
\end{tabular}




\subsection{Results from Model Regression of Experimental $\mathrm{NO}_{2}^{-}$Data}

The exchange current densities and the transfer coefficients for reactions (2-4) were determined with experiments of type 2 for a nickel cathode and stainless steel anode. Data from five experiments corresponding to experiments \#20, \#21, and \#26-\#28 were used with the model predictions to calculate the objective function of equation (38). Only the data at sample times corresponding to $150,180,210$, and 240 minutes were used from these experiments because it was observed that the data prior to these times corresponded to transient, non steady state behavior. Thus, even though the model can predict transient behavior, the determination of the best-fit kinetic parameters is more direct when steady state, uncorrelated data are used. A 3-D surface plot of this factorial design is shown in Figure 7. Details of the statistical analyses can be found by reference [15]. An outline of the kinetic parameters determined in the analysis of the data for the reduction of $\mathrm{NO}_{2}^{-}$is given in Table 8 .

In the analysis for the reduction of $\mathrm{NO}_{3}^{-}$, model predictions were compared to the experimental constant current density data of part I using the nonlinear least squares function (equation 39). In order to make accurate model predictions for the reduction of $\mathrm{NO}_{3}^{-}$, the exchange current densities and transfer coefficients of reactions (2-6) needed to be known within a high degree of certainty because there are two dependent variables in the least squares objective function. Model predictions were, therefore, obtained using the kinetic parameters of Table 8, which were calculated within $95 \%$ confidence intervals determined above and for reactions (2-6).

Previous estimates for the exchange current density $\left(i_{0,1}=1.0 \times 10^{-9} \mathrm{~A} / \mathrm{cm}^{2}\right)$ and transfer coefficient $\left(\alpha_{1}=0.4\right)$ for the reduction of $\mathrm{NO}_{3}^{-}$were made with the data from runs $5,8 \mathrm{a}, 8 \mathrm{~b}$, and 9. These values were taken as the median values and were changed in a more comprehensive $3^{2}$ factorial manner utilizing a step size of one order of magnitude for the exchange current density and 0.1 for the transfer coefficient. A surface plot of this analysis is shown in Figure 8. Details of statistical analyses can be found in reference [15]. Therefore, as determined by the experimental and numerical analyses, we have a complete set of exchange current densities and transfer coefficients for reactions (1-6) at a nickel cathode and stainless steel anode as shown in Table 8 . Note that the anodic reaction for oxygen generation was described in Section 2.

Table 8. Exchange Current Densities and Transfer Coefficients for Reactions (1-6), with $95 \%$ Confidence Intervals, using a Nickel Cathode and Stainless Steel Anode.

\begin{tabular}{|lcc|}
\hline & $\begin{array}{c}\text { Exchange Current Densities } \\
\left(\mathrm{i}_{0,1-6}\right), \mathrm{A} / \mathrm{cm}^{2}\end{array}$ & $\begin{array}{c}\text { Transfer Coefficients } \\
\alpha_{1-6}\end{array}$ \\
Reaction (1) & $2.0 \times 10^{-10} \pm 2.6 \times 10^{-12}$ & $0.4 \pm 1.1 \times 10^{-3}$ \\
Reaction (2) & $8.0 \times 10^{-07} \pm 1.9 \times 10^{-09}$ & $0.3 \pm 1.1 \times 10^{-3}$ \\
Reaction (3) & $2.0 \times 10^{-11} \pm 1.3 \times 10^{-11}$ & $0.3 \pm 4.1 \times 10^{-4}$ \\
Reaction (4) & $1.0 \times 10^{-11} \pm 5.2 \times 10^{-11}$ & $0.3 \pm 4.9 \times 10^{-05}$ \\
Reaction (5) & $1.0 \times 10^{-06} \pm 6.6 \times 10^{-09}$ & $0.635 \pm 2.4 \times 10^{-3}$ \\
Reaction (6) & $2.25 \times 10^{-04} \pm 5.1 \times 10^{-06}$ & $0.19 \pm 4.6 \times 10^{-3}$ \\
\hline
\end{tabular}




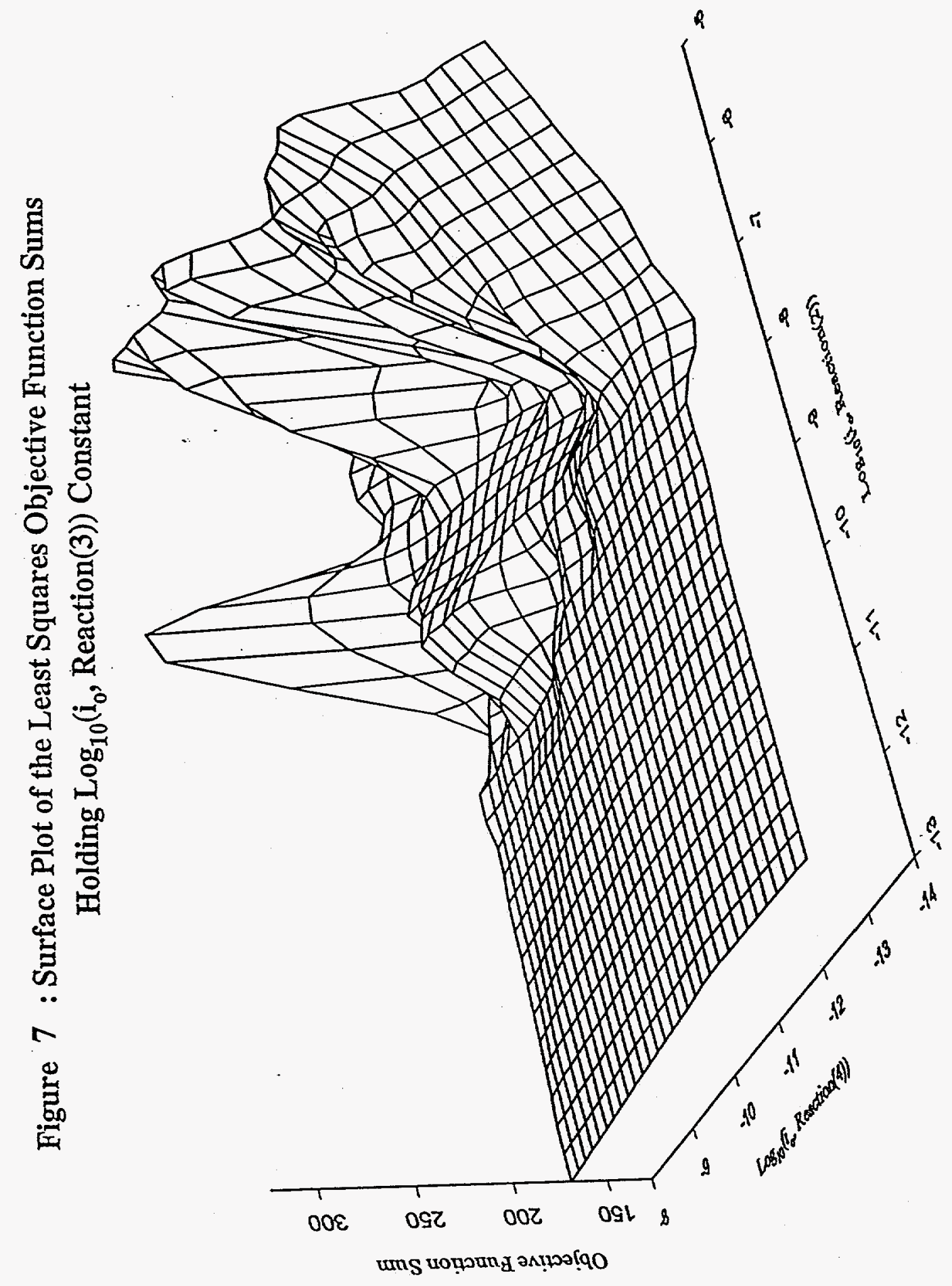


Figure 8 : Objective Function Sum as a Function of the Exchange

Current Density and Transfer Coefficient for the Reduction of Nitrate

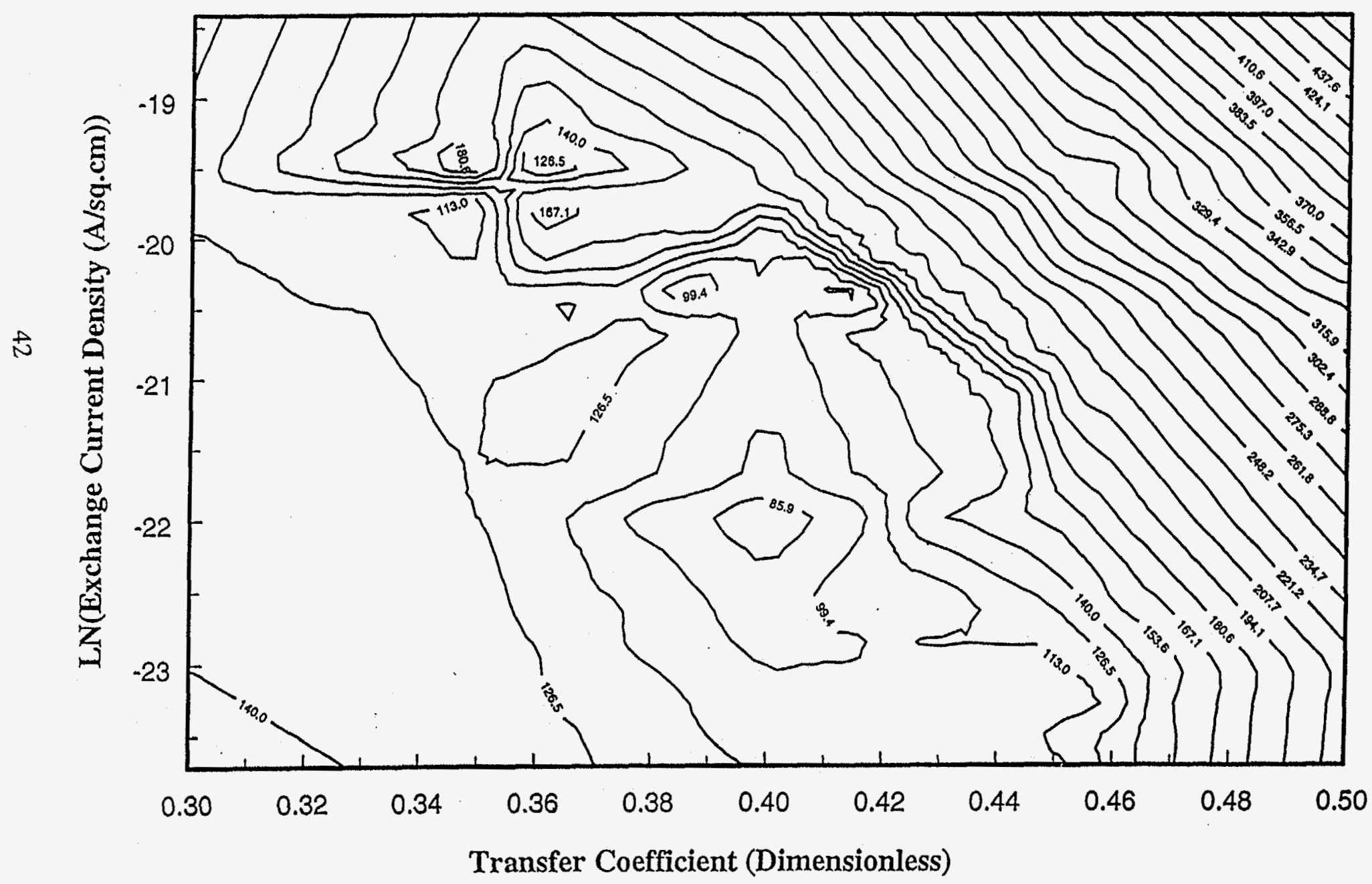


The calculation of the $95 \%$ confidence intervals for the exchange current densities and transfer coefficients of reactions (1-4) in Table 8 are outlined in Appendix K of Ref 15.

Note that a high degree of confidence can be placed in the values for reactions (1) and (2) due to large changes in the observed concentrations of $\mathrm{NO}_{2}^{-}$and $\mathrm{NH}_{3}$. On the hand, there is a low degree of confidence on the values of reactions (3) and (4) due to small molar off-gas flow rates of $\mathrm{N}_{2}$ and $\mathrm{N}_{2} \mathrm{O}$.

\subsection{Residual Plots}

Residual plots of the dependent variables of the nonlinear least squares objective functions are shown in Figures $9-12$ at the statistically determined exchange current densities and transfer coefficients outlined in reactions (2-4) of Table 8. These dependent variables include the $\mathrm{NH}_{3}$ concentration in the catholyte in Figure 9, the $\mathrm{N}_{2}$ off-gas molar flow rate in Figure 10, the $\mathrm{N}_{2} \mathrm{O}$ off-gas molar flowrate in Figure 11, and the cathodic potential in Figure 12. The observations are separated by experiment run number in each figure. Also, residual plots of the dependent variables of equation (41) of Chapter 3 are shown in Figures 13 and 14 corresponding to the $\mathrm{NO}_{2}^{-}$ concentration in the catholyte and the cathodic potential respectively. Figures 9 through 14 are defined in terms of the experimentally observed minus the predicted values. It is difficult to make any broad statements about the data shown to the limited number of observations (i.e., 19 in Figures $9-12$ and 24 in Figures 13 and 14). However, Figure 9 shows a bias toward the under prediction of the experimental data in experiment run 26. In Figure 10, experiment runs 20 and 28 show a bias toward the under prediction of the experimental data. Figure 11 shows exceptional agreement between the observed and predicted values of the $\mathrm{N}_{2} \mathrm{O}$ off-gas molar flow rate. Again, it is important to note that only small amounts of $\mathrm{N}_{2} \mathrm{O}$ were observed in our experiments. The residuals of Figure 12 for the cathodic potential separate into groups corresponding to the current density $\left(I=0.15 \mathrm{~A} / \mathrm{cm}^{2}\right)$ at predicted cathodic potentials between 1.04 and 1.06 Volts, and $\left(I=0.25 \mathrm{~A} / \mathrm{cm}^{2}\right)$ at cathodic potentials between 1.085 and 1.105 Volts. Although, the absolute difference may be as large as 0.3 Volts, the residuals do not show an obvious correlation with current density. Further experimental work should concentrate on replicates of this measurement.

Figures 13 and 14 correspond to the dependent variables for the reduction of $\mathrm{NO}_{3}^{-}$. Figure 13 shows a bias for the under prediction of the concentration of $\mathrm{NO}_{2}^{-}$in the catholyte in experiment run 29 . The residuals of the cathodic potential predictions shown in Figure 14 show an apparent normal distribution. 


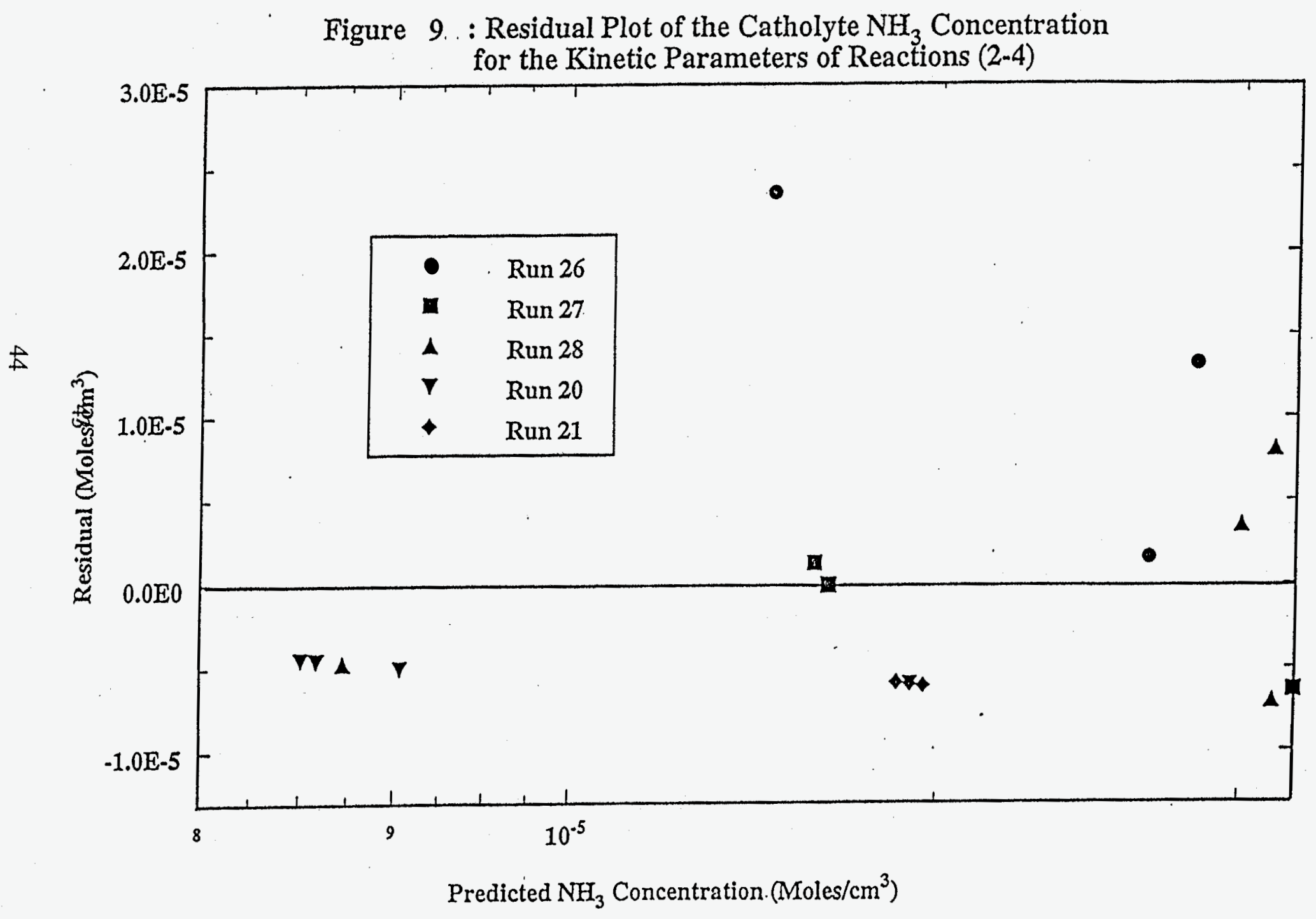


Figure 10 : Residual Plot of the $\mathrm{N}_{2}$ Off-Gas Flow Rate for the Kinetic Parameters of Reactions (2-4)

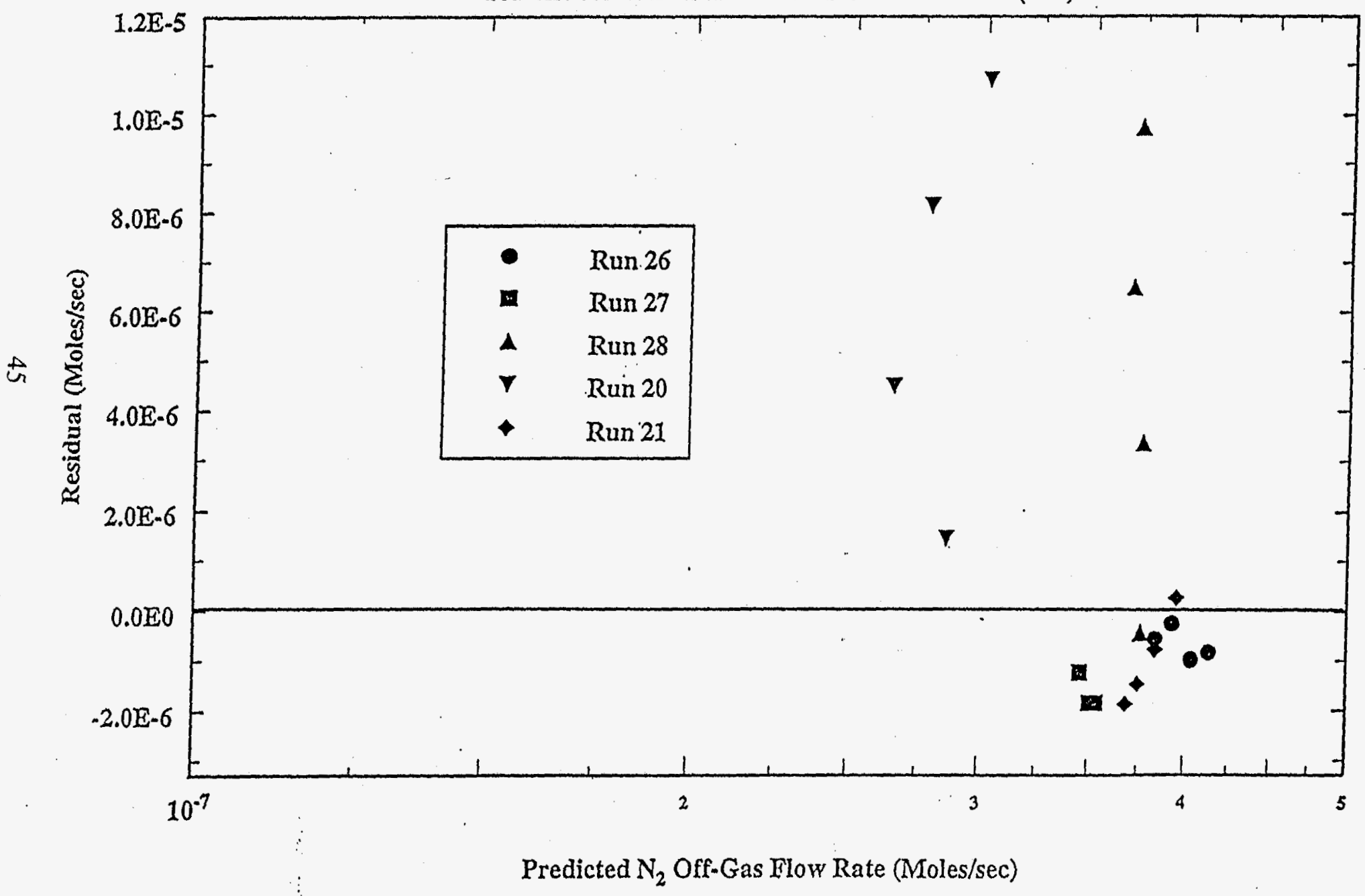




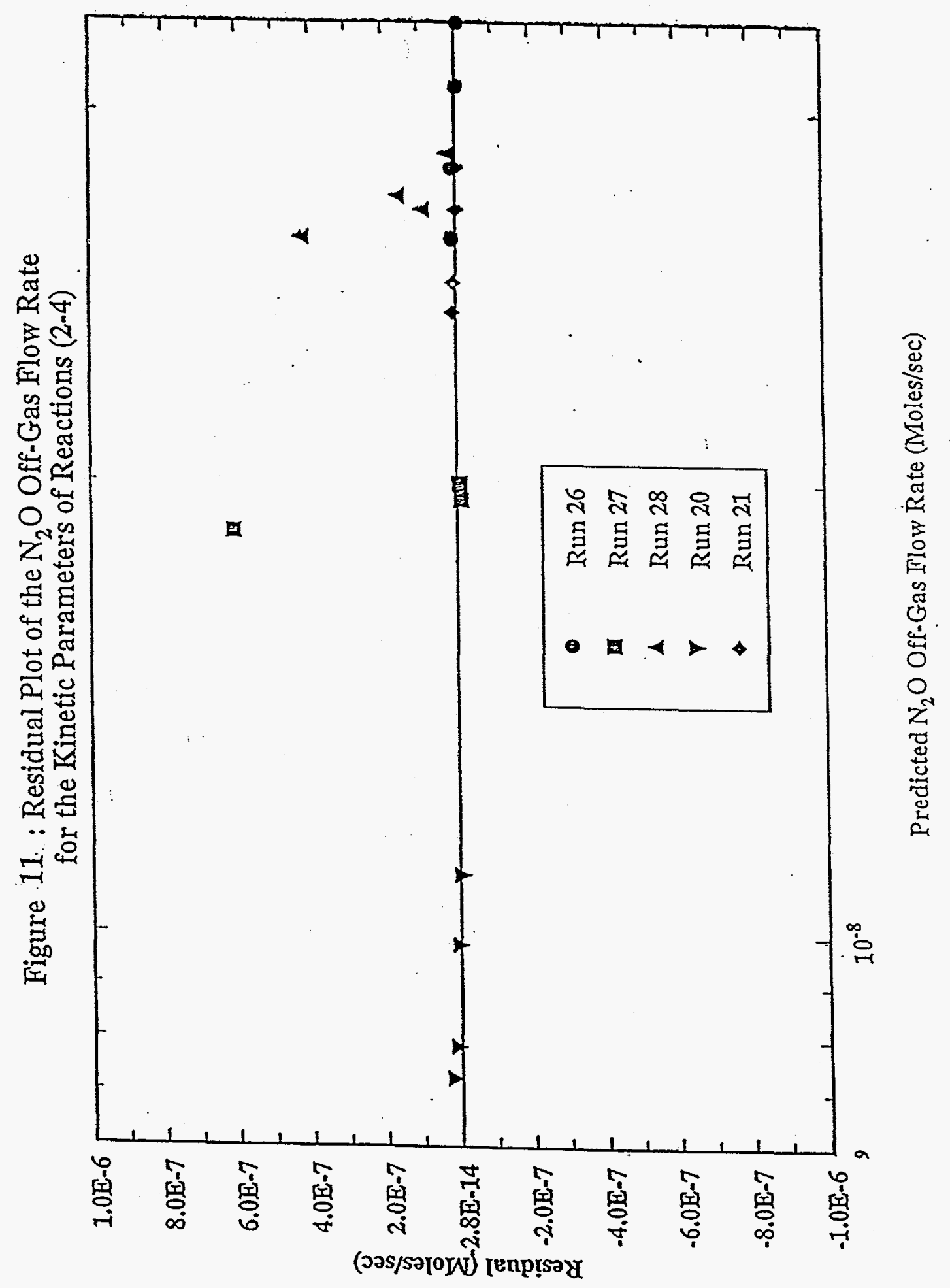


Figure 12 : Residual Plot of the Cathodic Potential for the Kinetic Parameters of Reactions (2-4)

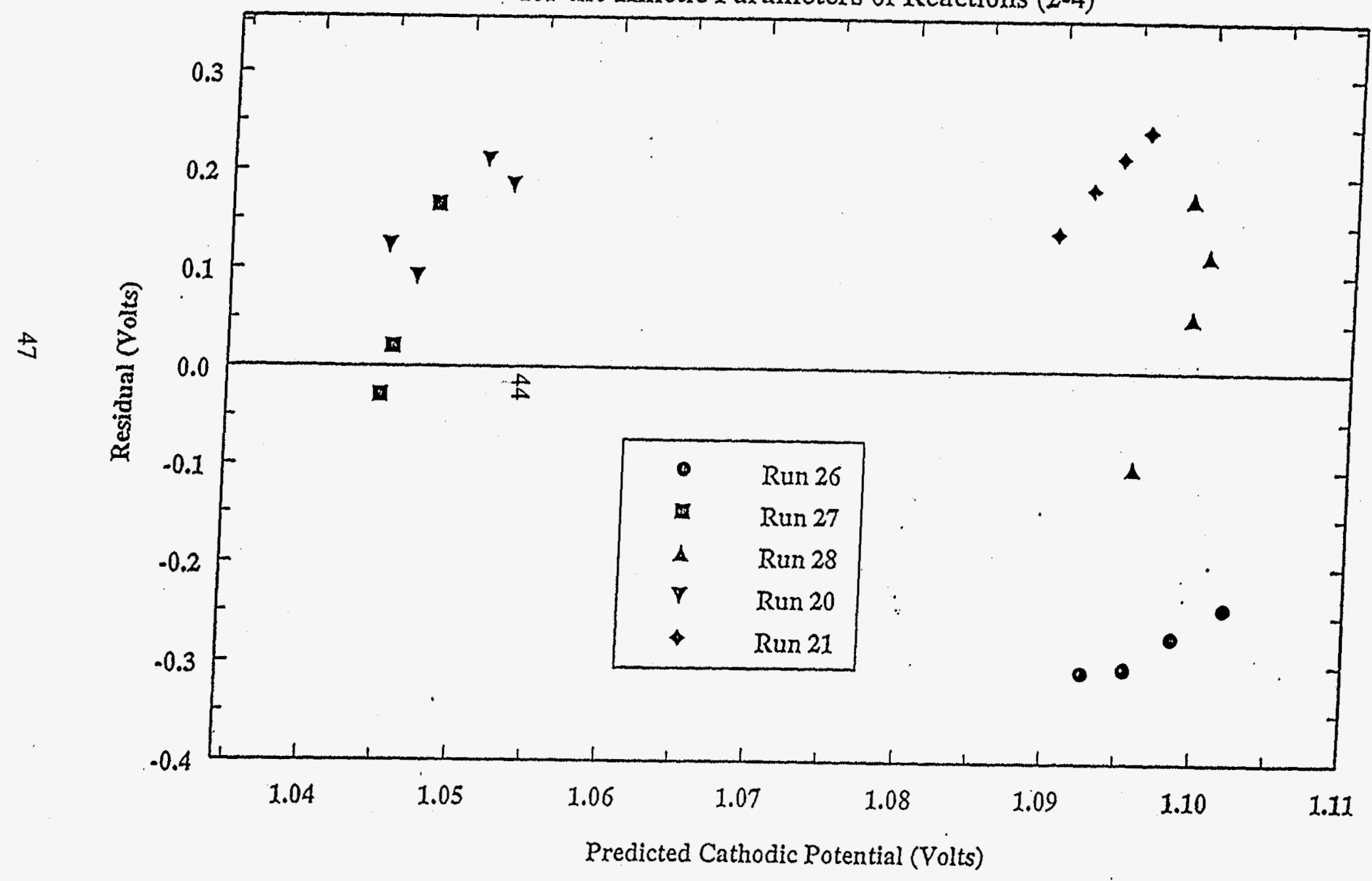




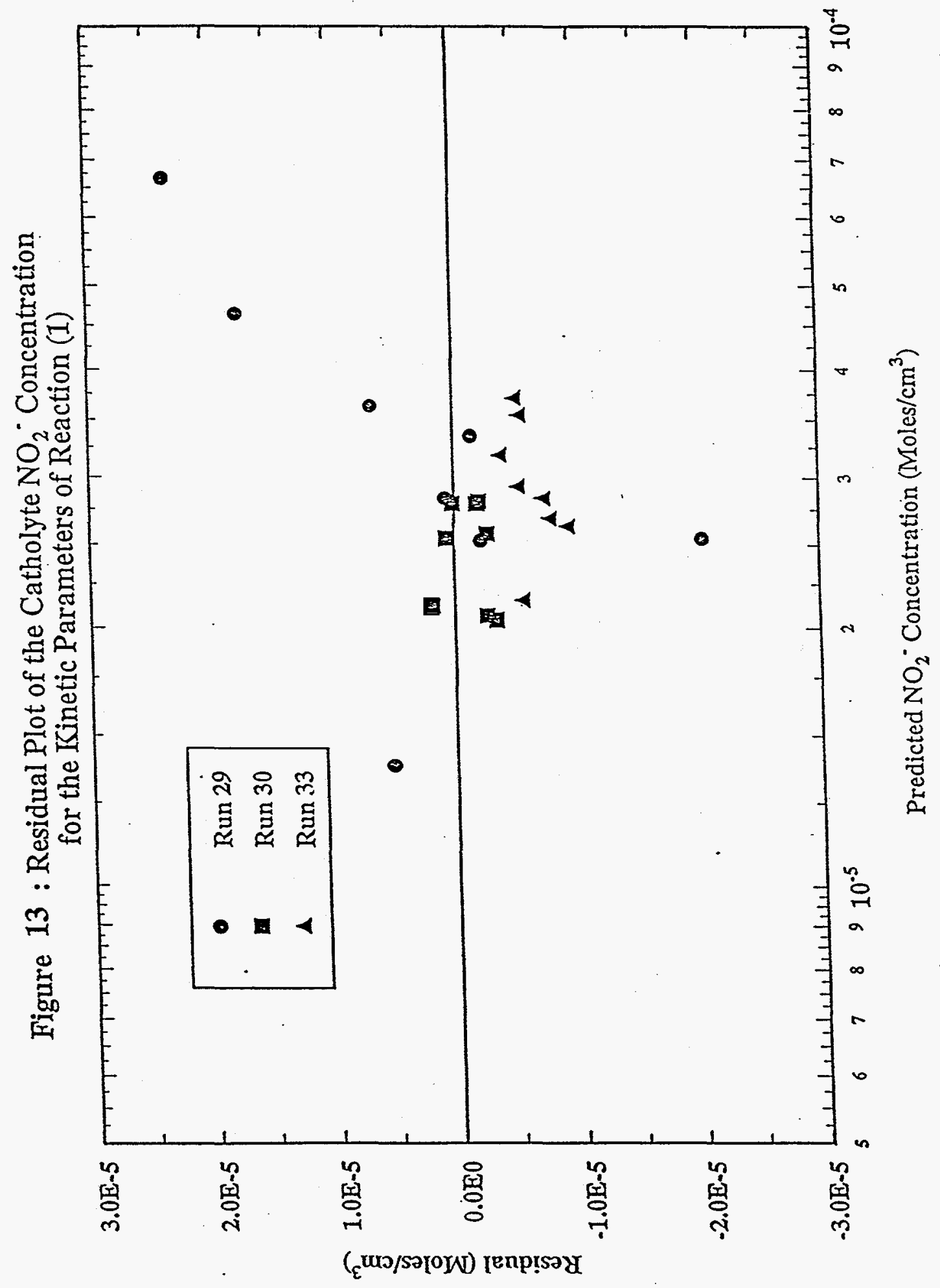




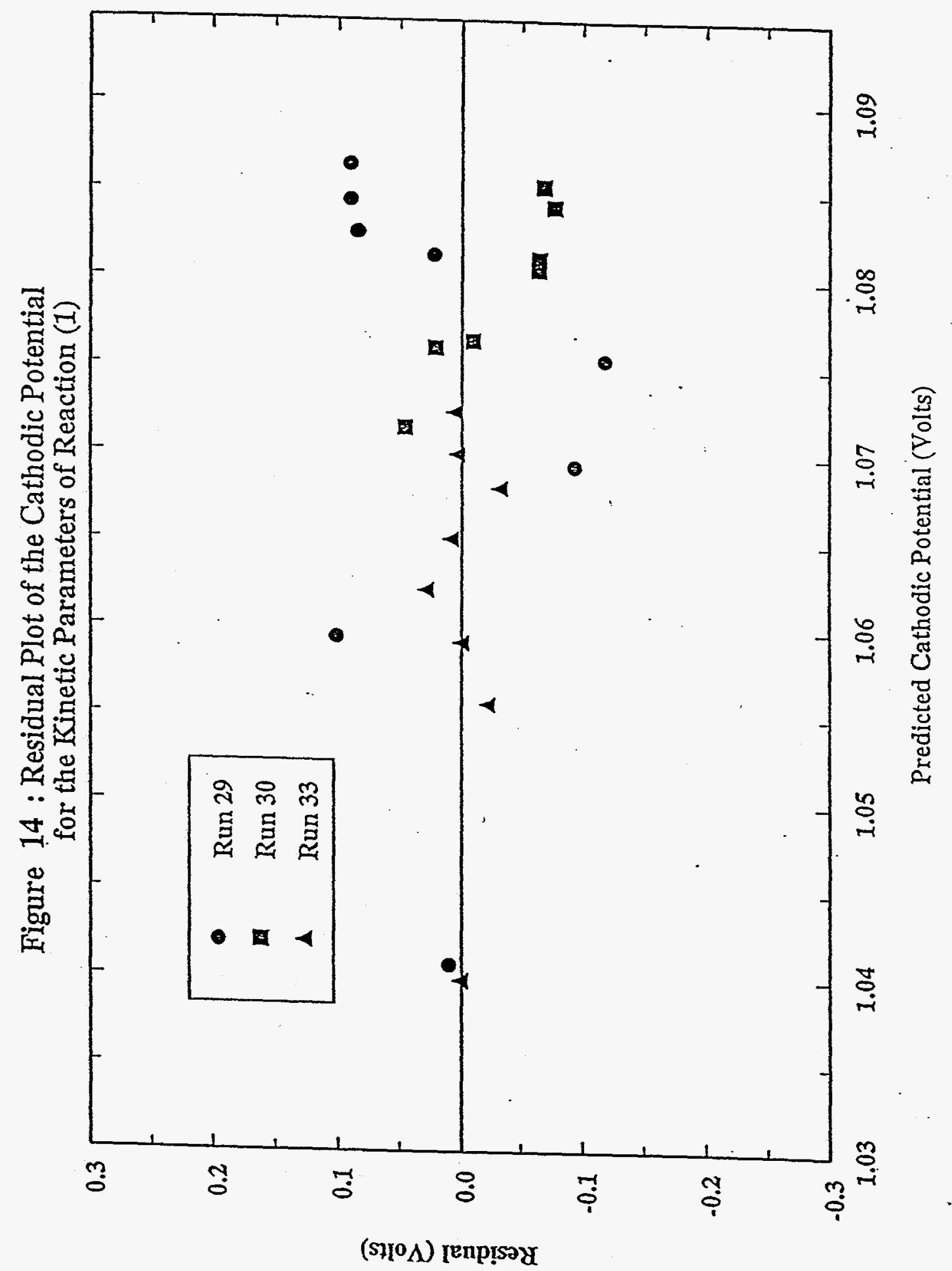




\subsection{Introduction}

\subsection{Model Predictions}

In this section, the model and the kinetic parameters are compared with new data from the batch reactor. The results show good agreement for a case where the current density is changed to minimize the water reduction reaction. Thus in this section, the concept of optimized electrolyzer operation is demonstrated by adjusting the current to maximize the current efficiency for the reduction of $\mathrm{NO}_{3}{ }^{-}$and $\mathrm{NO}_{2}^{-}$while minimizing the reduction of $\mathrm{H}_{2} \mathrm{O}$. This optimization is sensitive to the temperature of the electrochemical process. Also, the optimization is sensitive to type of electrocatalyst in so much as the values of the partial exchange current densities for the nitrate and nitrite reactions depend on the electrocatalyst. That is even though we present data for only one type of cathode, one would expect a different adjustment protocol if another electrode yields different electrochemical kinetics.

\subsection{Experiments}

Verification of the numerical model is presented here using the experiments of Type 4 of Section 2. Experiments were conducted in which full simulant (FS) concentrations (i.e., concentrations of $1.9 \mathrm{M} \mathrm{NaNO}_{3}, 0.6 \mathrm{M} \mathrm{NaNO}_{2}$, and $1.3 \mathrm{M} \mathrm{NaOH}$ ) were electrochemically reduced to $95 \%$ destruction of the total $\mathrm{NO}_{3}{ }^{-}$and $\mathrm{NO}_{2}{ }^{-}$concentration (i.e., $0.125 \mathrm{M}^{\text {total }} \mathrm{NO}_{3}{ }^{-}$ and $\mathrm{NO}_{2}{ }^{-}$). These batch experiments were performed for approximately 25 hours at a constant current density, with starting concentrations of $1.9 \mathrm{M} \mathrm{NaNO}_{3}, 0.6 \mathrm{M} \mathrm{NaNO}_{2}$, and $1.3 \mathrm{M}$ $\mathrm{NaOH}$ in the catholyte and 5.0 M NaOH in the anolyte. Also batch experiments were performed where the current density was changed from $0.25 \mathrm{~A} / \mathrm{cm}^{2}$ to $0.05 \mathrm{~A} / \mathrm{cm}^{2}$.

These FS experiments were conducted in accordance with the numerical model predictions which were used to determine the initial and operating conditions (i.e., the initial anolyte and catholyte volumes, the required GC carrier gas flow rate, off-gas compositions and flow rates, and the concentration changes in the ionic species in the anolyte and catholyte) required to achieve $95 \%$ destruction of the $\mathrm{NO}_{3}{ }^{-} / \mathrm{NO}_{2}^{-}$. The kinetic parameters determined for reactions $(1-6)$ were then used in the model predictions of the FS runs. These predictions were compared to the experimental data to verify the numerical boundary-layer model.

Early experiments in the analysis of this process consisted of full simulant batch runs (2 and 3 in Table 3-3 of Ref. 15) which were conducted over three to six day periods for a total electrolysis time of 20 hours. The experiments were conducted at a constant current density and electrolyte flow rate of $1 \mathrm{gpm}$. This data did not include temperature measurement, Kjeldahl analysis, volume change measurements, off-gas GC analysis, or off-gas flow rate measurement. However, the experiments did include measurements of the total cell potential and the concentrations of the anodic and cathodic species as a function of charge passed. The data for these experiments is shown in Appendix B.

\subsection{Model Predictions}

Model predictions of the concentration changes in the anolyte and catholyte are shown as a function of charge passed in Figure 15. These model predictions were obtained utilizing the kinetic parameters presented in Table 8 which were determined in the experimental analysis of Section 2 and the statistical analysis of Section 4. The physical and mass transport 


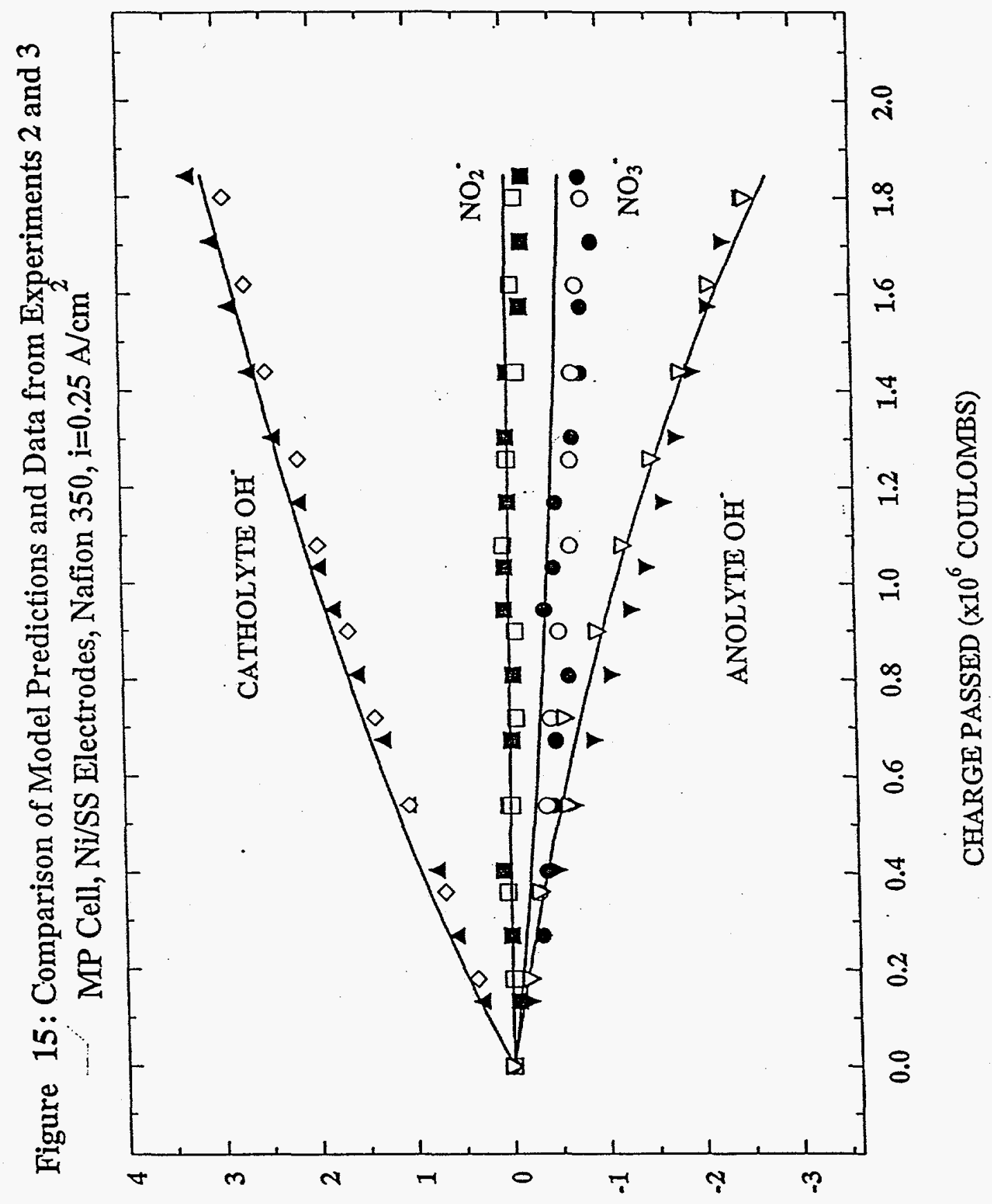

(T/SHTOW) HDNVHO NOILVAINADNOD 
parameters used in the model predictions are shown Table 9.

Table 9. Physical and Mass Transfer Parameters for Experiments 2 and 3 using a Nickel Cathode and Stainless Steel Anode in an MP cell.

\begin{tabular}{|c|c|}
\hline 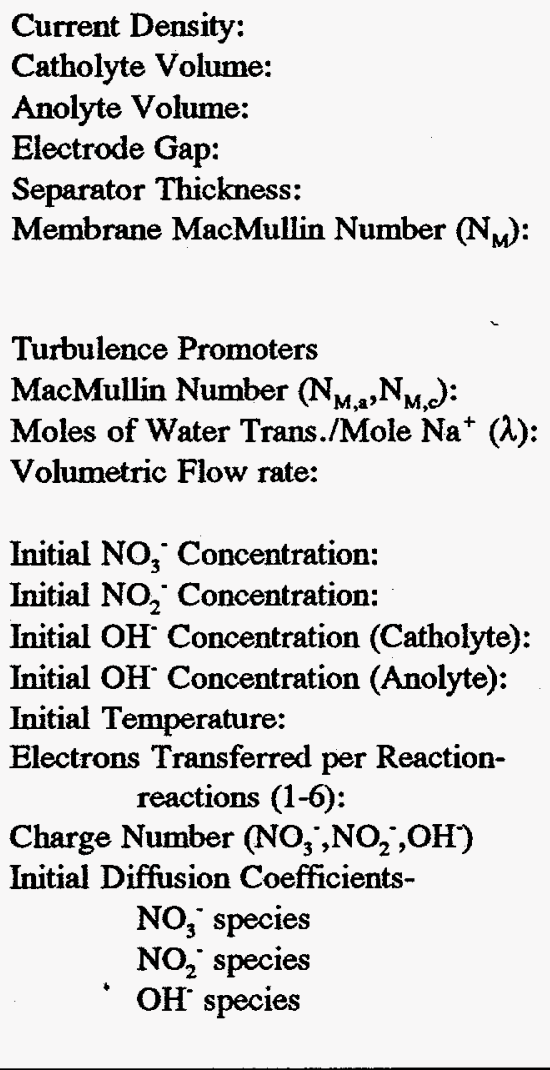 & $\begin{array}{l}8.25 \mathrm{~A} / \mathrm{cm}^{2} \\
8000 \mathrm{ml} \\
0.6 \mathrm{~cm} \\
0.05 \mathrm{~cm} \\
\mathrm{~N}_{\mathrm{M}}=44.4 @ 21^{\circ} \mathrm{C} \\
\mathrm{N}_{\mathrm{M}}=18.7 @ 40^{\circ} \mathrm{C} \\
\left.\mathrm{N}_{\mathrm{M}}=(-1.35) \mathrm{T} \mathrm{C}^{\circ} \mathrm{C}\right)+72.7 \\
\mathrm{~N}_{\mathrm{M}, \mathrm{a}}=\mathrm{N}_{\mathrm{M}, \mathrm{c}}=2.40 \\
10.0 \\
10.5 \mathrm{~cm}^{3} / \mathrm{sec} \\
\text { Exp.2 } 2 \text { Exp. } 3 \\
2.01 \times 10^{-3} 1.82 \times 10^{-3} \mathrm{moles} / \mathrm{cm}^{3} \\
0.60 \times 10^{-3} \quad 0.53 \times 10^{-3} \mathrm{moles} / \mathrm{cm}^{3} \\
1.40 \times 10^{-3} \quad 1.18 \times 10^{-3} \mathrm{moles} / \mathrm{cm}^{3} \\
5.71 \times 10^{-3} \quad 4.85 \times 10^{-3} \mathrm{moles} / \mathrm{cm}^{3} \\
25^{\circ} \mathrm{C} \\
1 \\
-1 \\
1.902 \times 10^{-5} \mathrm{~cm}^{2} / \mathrm{sec} \\
1.902 \times 10^{-5} \mathrm{~cm}^{2} / \mathrm{sec} \\
5.260 \times 10^{-5} \mathrm{~cm}^{2} / \mathrm{sec} \\
\mathrm{D}_{\mathrm{k}} \mu / \mathrm{T}=\mathrm{constant}^{2}\end{array}$ \\
\hline
\end{tabular}

A later experimental full simulant run was conducted on 10/11 - 10/12/95 (i.e., experiment \#38) which was rigorous in the experiment design and the collection of data. This data is summarized in Appendix B. Liquid samples of the anolyte and the catholyte were collected at two hour intervals for the duration of the experiment. Gas samples and flow rate measurements of the catholyte were performed at one hour intervals. The catholyte reservoir and cell, and the anolyte reservoir and cell temperature were measured at one hour intervals. Potential differences between the anode and the anode reference, the anode reference and the cathode reference, the cathode reference and the cathode, and the total potential difference between the anode and the cathode were measured at one hour intervals. With a nominal catholyte concentration of $1.95 \mathrm{M} \mathrm{NaNO}_{3}, 0.60 \mathrm{M} \mathrm{NaNO}_{2}$, and $1.33 \mathrm{M} \mathrm{NaOH}$, chloride was added to determine volume changes in the catholyte by ion chromatography analysis. The initial conditions and the physical and mass transport parameters used in the numerical model predictions of this experimental data are shown in Table 10. 
Table 10. Initial Conditions, Physical and Mass Transfer Parameters for Experiment \#38 using a Nickel Cathode and Stainless Steel Anode in an MP cell.

\begin{tabular}{|c|c|}
\hline 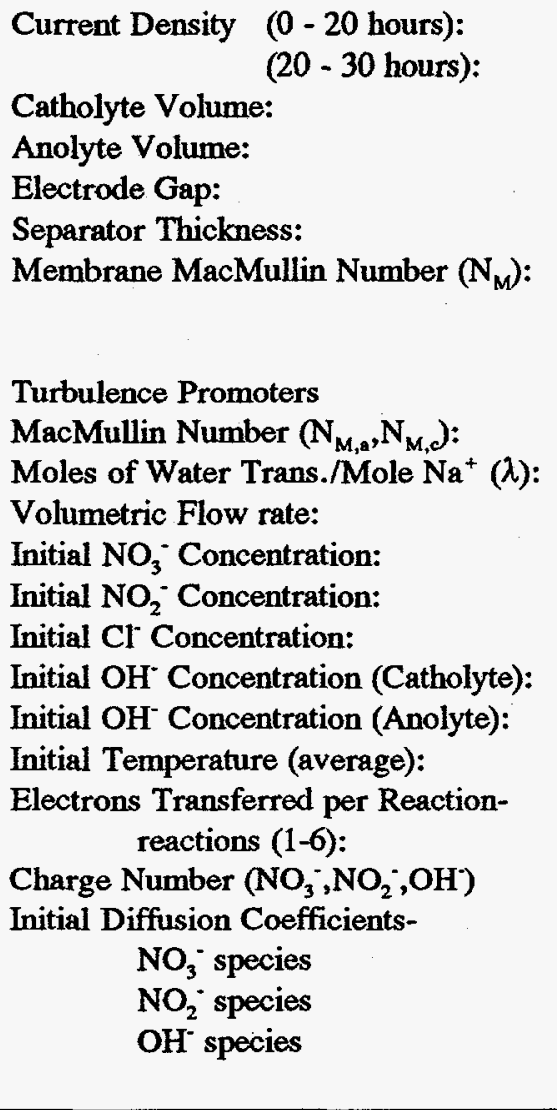 & 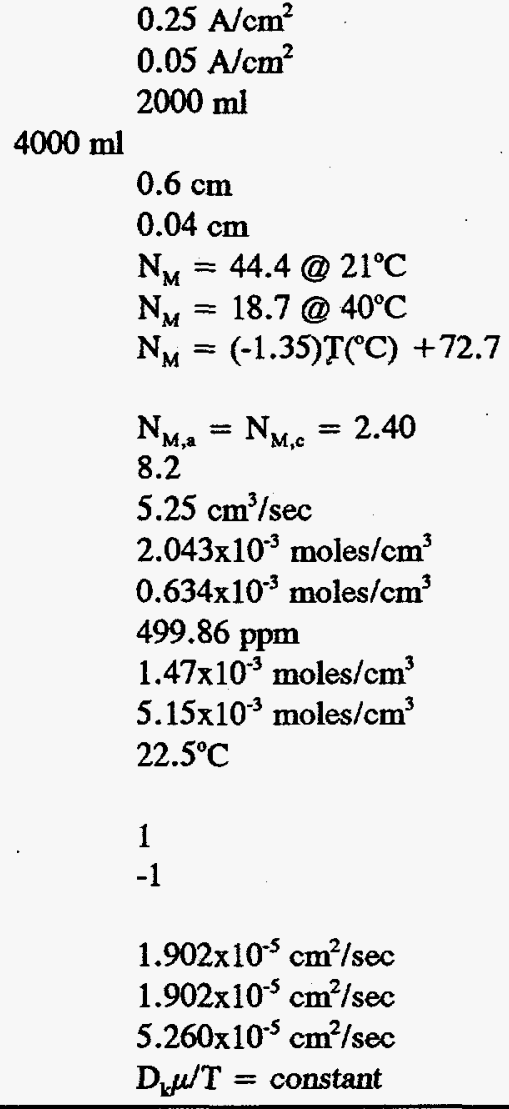 \\
\hline
\end{tabular}

The exchange current densities and transfer coefficients used in the model predictions were again those taken from Table 8. Model predictions of the concentration changes of species: $\mathrm{NO}_{3}^{-}, \mathrm{NO}_{2}^{-}, \mathrm{NH}_{3}$, and $\mathrm{OH}^{-}$in the catholyte and $\mathrm{OH}^{-}$in the anolyte as a function of time are shown in Figure 16. The variation between the model predictions of the $\mathrm{OH}^{-}$ concentration in the anolyte and the catholyte is likely due to the effects of migration and diffusion of the $\mathrm{OH}^{-}$species. This could not be verified experimentally and $\lambda$ could be considered as an adjustable parameter. For example, the responsiveness of $\mathrm{OH}^{-}$concentration changes in the catholyte to the moles of $\mathrm{H}_{2} \mathrm{O}$ transported into the catholyte per mole of $\mathrm{Na}^{+}$ $(\lambda)$ is illustrated in Figure 17. Model predictions of $\mathrm{OH}^{-}$changes in the anolyte are superimposed in the figure which is due the large volume of the anolyte (i.e., approximately 4 to 4.5 liters during the experiment).

Model predictions of the catholyte volume versus experimental data as a function of time are shown in Figure 18. The jagged step-wise manner that the model predictions and the experimental data follow is due to the large catholyte sample size requirements at two hour intervals (i.e., $100 \mathrm{ml}$ of catholyte) for ion chromatography $\left(\mathrm{NO}_{3}^{-}, \mathrm{NO}_{2}^{-}\right.$, and $\mathrm{Cl}^{-}$), titration 


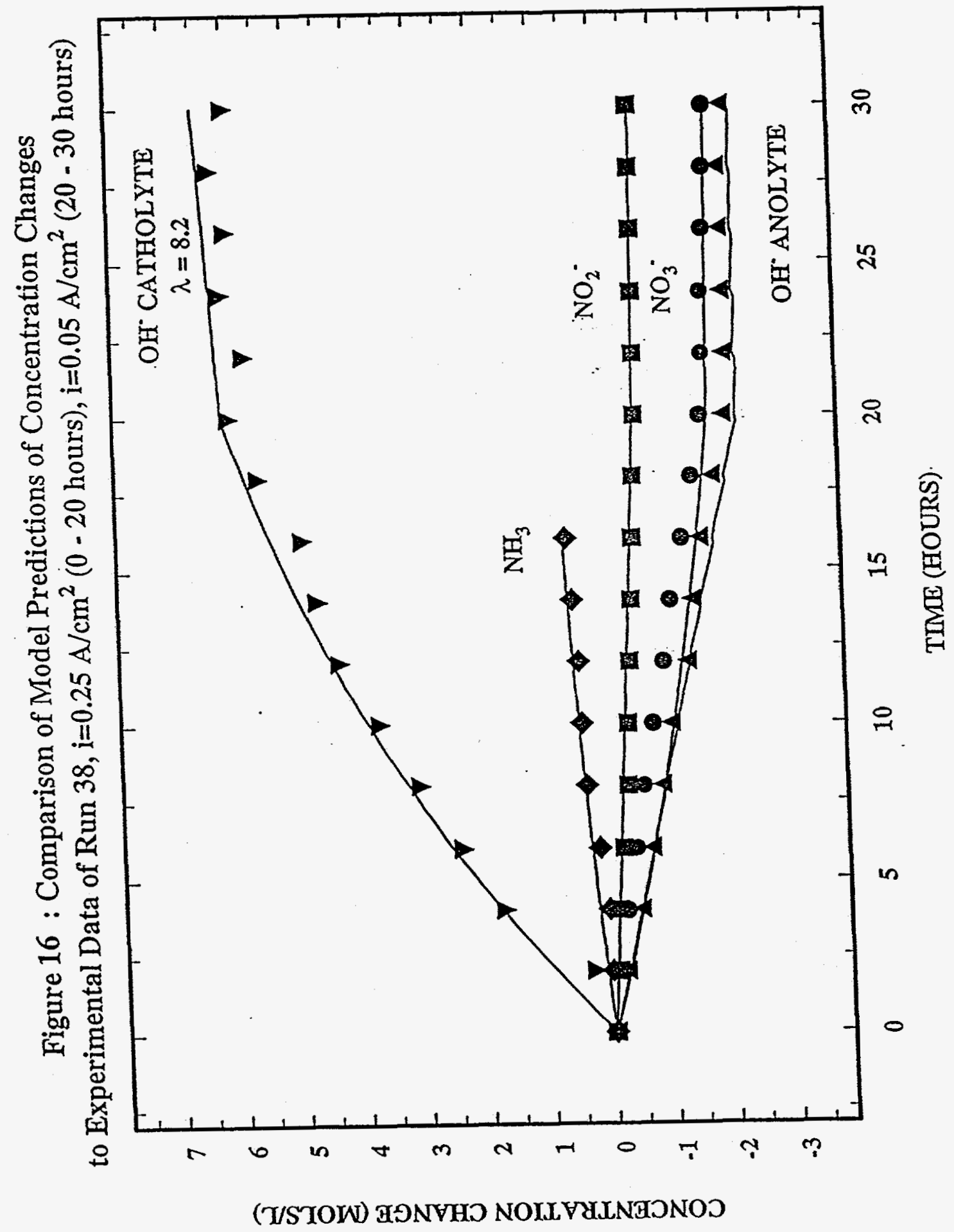


Figure 17: Comparison of the Effects of Water Transport on Concentration Changes in the Anolyte and the Catholyte

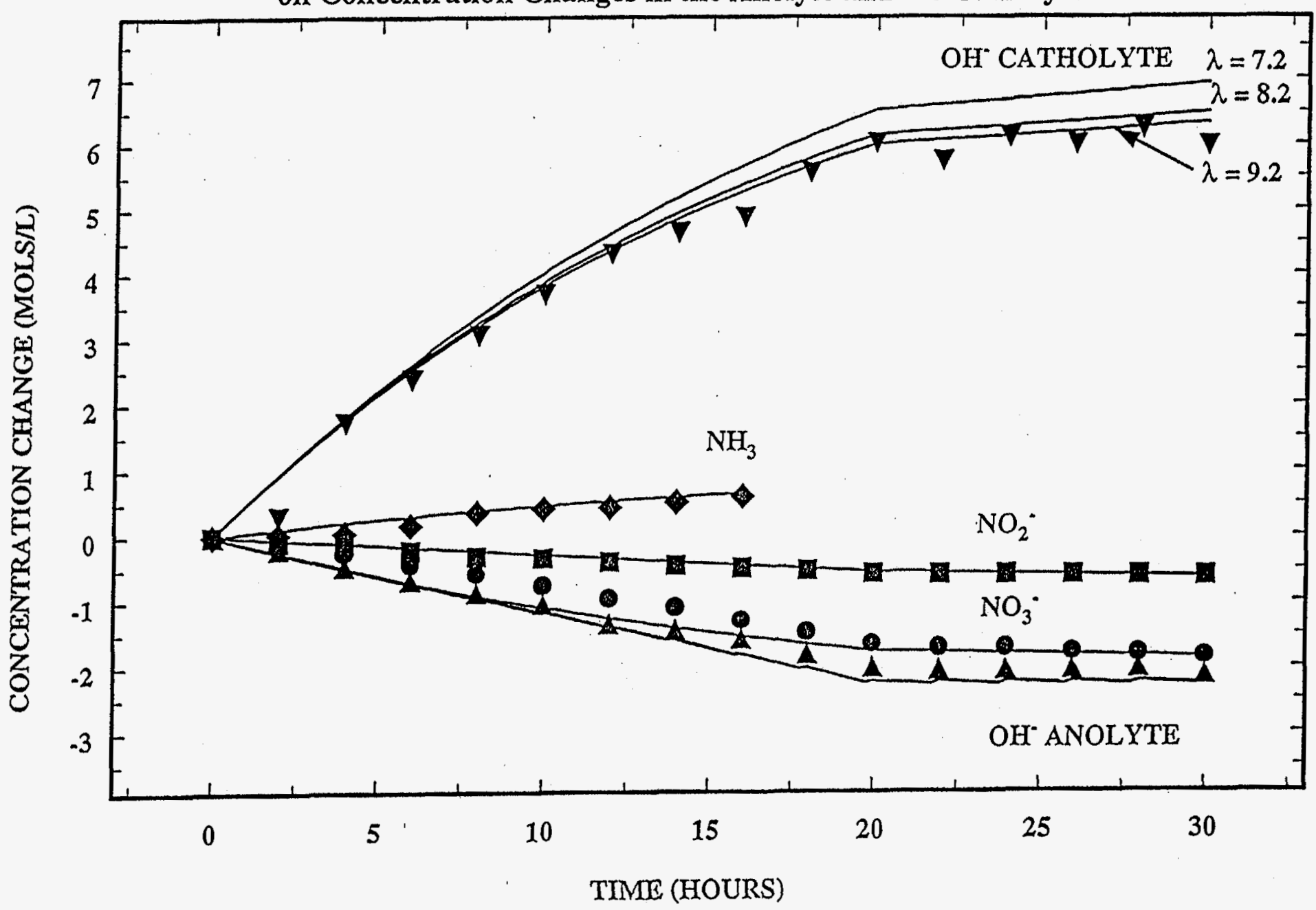




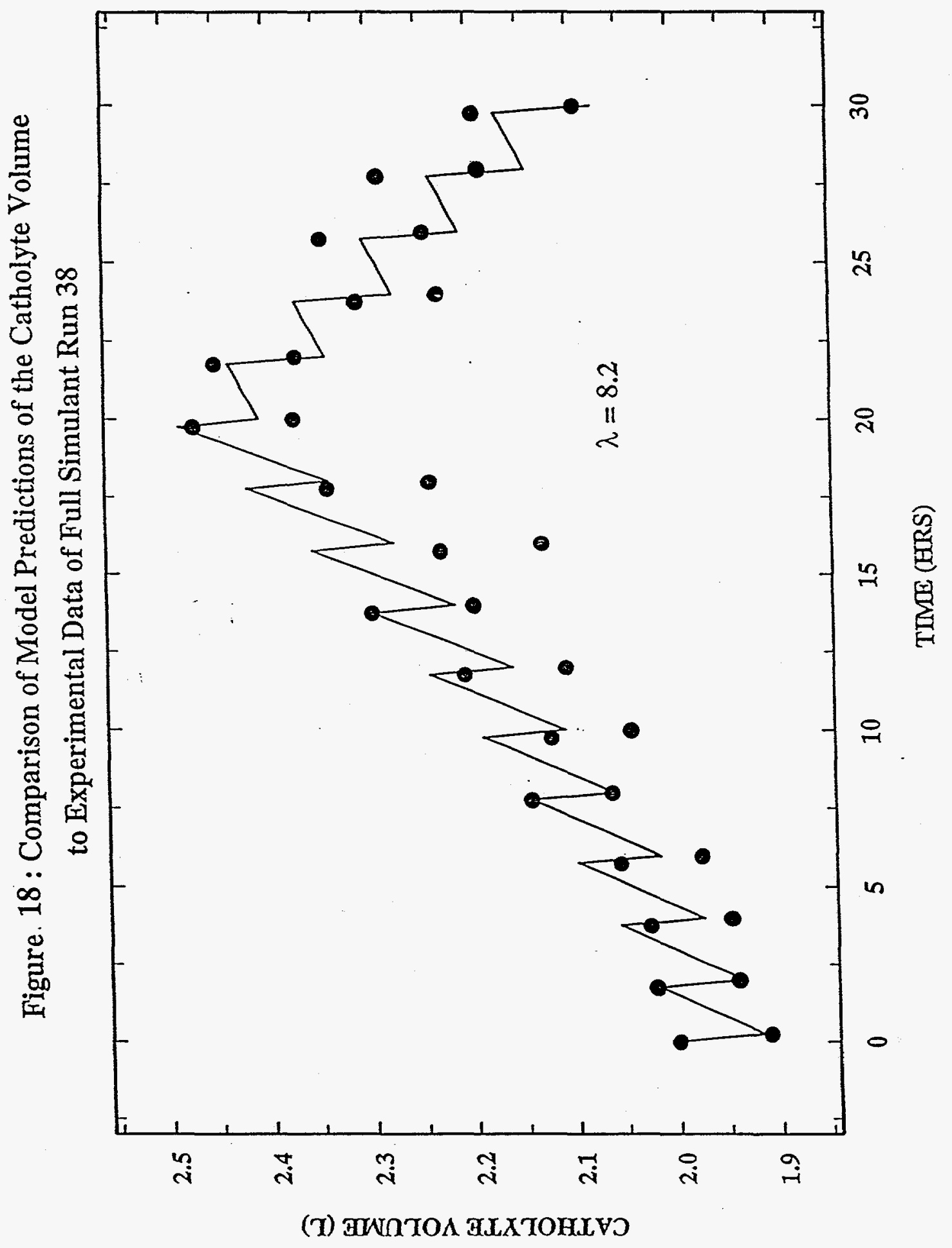


$\left(\mathrm{OH}^{-}\right)$, and $\mathrm{Kjeldahl}$ analysis $\left(\mathrm{NH}_{3}\right)$. The catholyte volume increases during the first 20 hours of the experiment. This is caused by the flux of water into the catholyte due to the high current density $\left(0.25 \mathrm{~A} / \mathrm{cm}^{2}\right)$. The model equations account for this with $\lambda$ and in Figure 18 we use a value of $\lambda=8.2$ as discussed below. After 20 hours, the catholyte volume decreases with time which due to the decreased flux of water across the membrane. The flux of water into the catholyte is less than the volume removed by sampling which results in a decreasing catholyte volume. The sensitivity of the changes in catholyte volume to $\lambda$ is shown in Figure 19. As seen in the figure, a change in $\lambda$ of 1 results in approximately a $120-\mathrm{ml}$ change in the final catholyte volume. A value of $\lambda=9.2$ tends to over predict the catholyte volume after 22 hours and a value of $\lambda=7.2$ tends to under predict the volume as shown in Figure 19. Thus we estimate that $\lambda=8.2$ for the membrane used in these experiments. Future work should include experiments to determine if this value is constant with the lifetime of the membrane.

Model predictions versus experimental data and the relationship between the total cell potential and temperature is illustrated in Figure 20. As shown in the figure, the cell potential decreases with increasing temperature and thus, increasing electrolyte conductivity and decreasing resistance. The cell undergoes a rapid temperature rise on start-up that goes through a slight maximum before achieving steady-state conditions. This maximum is due to a slow rate of radiant heat transfer. The total cell potential drops sharply when the current density is decreased. This is due to the direct proportionality between current density and cell potential as seen in equation (5) of Section 3. The slight rise in the temperature of the process toward the end of the $0-20$ hour period at a current density of $0.25 \mathrm{~A} / \mathrm{cm}^{2}$ is due to an increasing current efficiency for the hydrogen generation reaction and the onset of gassing conditions. The sensitivity of the hydrogen generation reaction to the kinetic parameters (i.e., $\alpha_{5}$ and $i_{05}$ ) is discussed below.

Model predictions of the off-gas composition from the catholyte demonstrated that the contribution from $\mathrm{N}_{2}$ and $\mathrm{N}_{2} \mathrm{O}$ was minor. This was verified experimentally as shown in Figure 21. The rise in the experimental off-gas composition of $\mathrm{H}_{2}$ could not be predicted numerically. Model predictions deviate from the experimental data at approximately 10 hours into the run as shown in the figure. This is most likely a result of dead space where the Ar purge did not enter and thus there was an accumulation of $\mathrm{H}_{2}$ in the space above the catholyte liquid. Not that the system had argon gas flowing at a constant rate of $5 \mathrm{ml} / \mathrm{min}$ during the experiment. Other factors which affect the experimental data include a changing generation rate of $\mathrm{H}_{2}$ and changing catholyte volume throughout the full simulant run. Also, model predictions of the current efficiency for the hydrogen generation reaction are highly sensitive to small changes in the transfer coefficients and exchange current densities (i.e., $\alpha_{5}$ and $i_{o s}$ ). This sensitivity is illustrated to Figure 22 in which $i_{o 5}$ is held constant while $\alpha_{5}$ is varied between $0.6,0.635$, and 0.7 . Model predictions of the off-gas mole fraction of $\mathrm{H}_{2}$ increases approximately $26 \%$ corresponding to an increase in $\alpha_{5}$ from 0.6 to 0.7 . Although we believe dead-space accumulation is the cause of the deviations between the model and the data, future work might investigate the change in these parameters with time. Figure 23 illustrates the response of the off-gas percentage of $\mathrm{H}_{2}$ to changes in $\mathrm{i}_{\mathrm{os}}=1.0 \times 10^{-6} \mathrm{~A} / \mathrm{cm}^{2}$ of one-half order of magnitude above and below for a constant $\alpha_{5}$.

Overall, the comparison of the model predictions to experimental data showed 


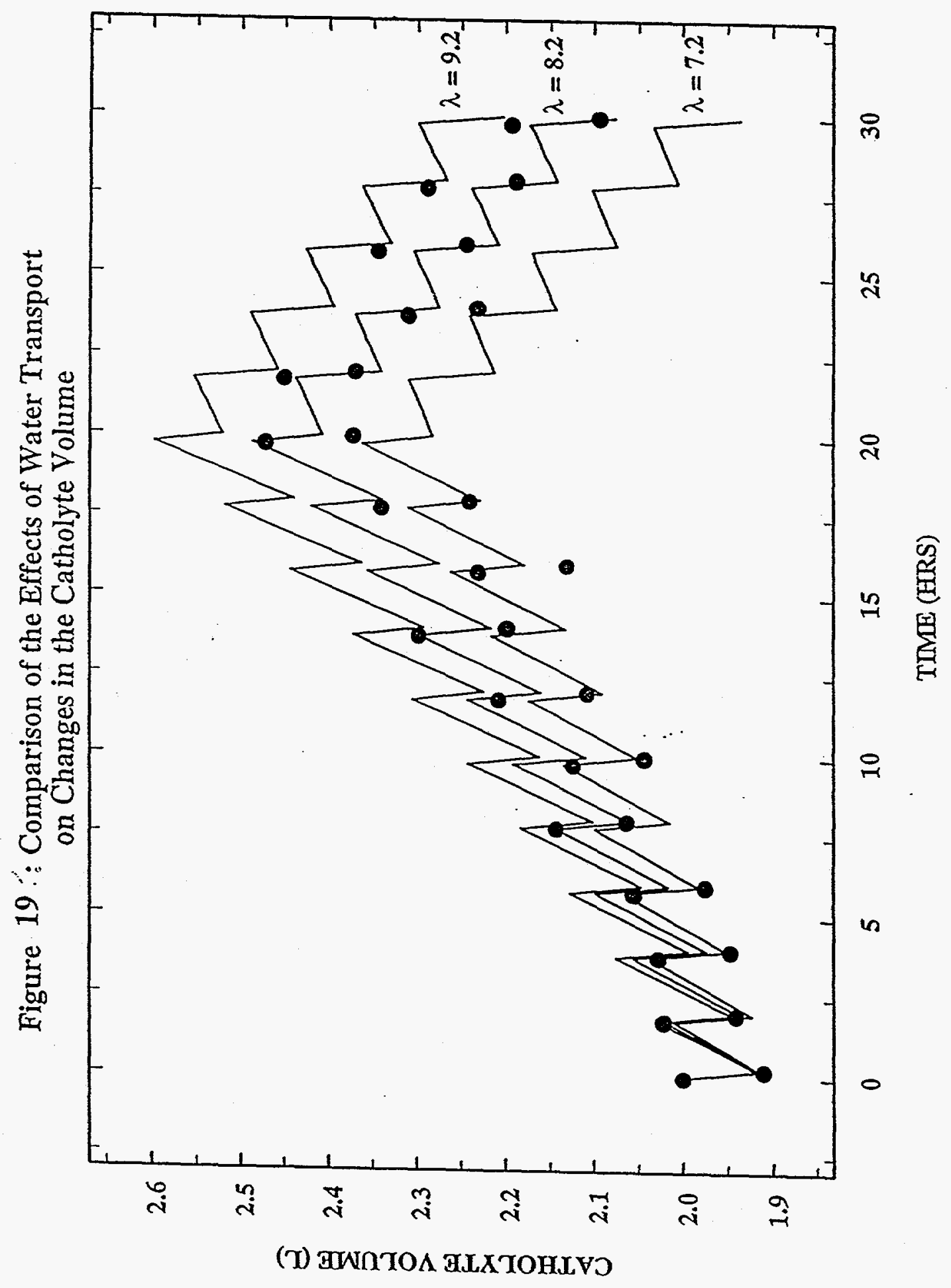




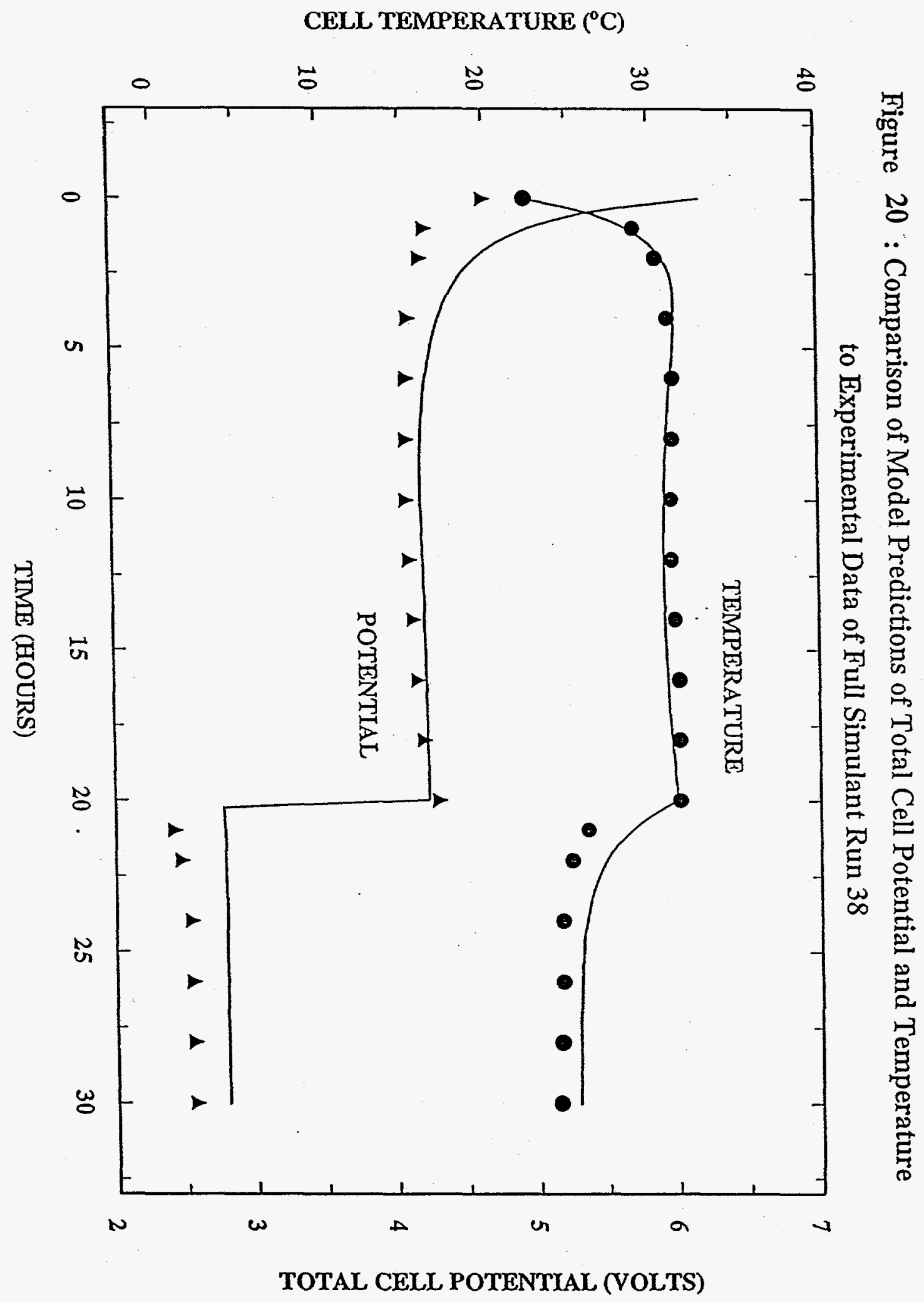


Figure 2i: Comparison of Model predictions of Off-Gas Molar Percentages to Experimental Data of Full Simulant Run 38

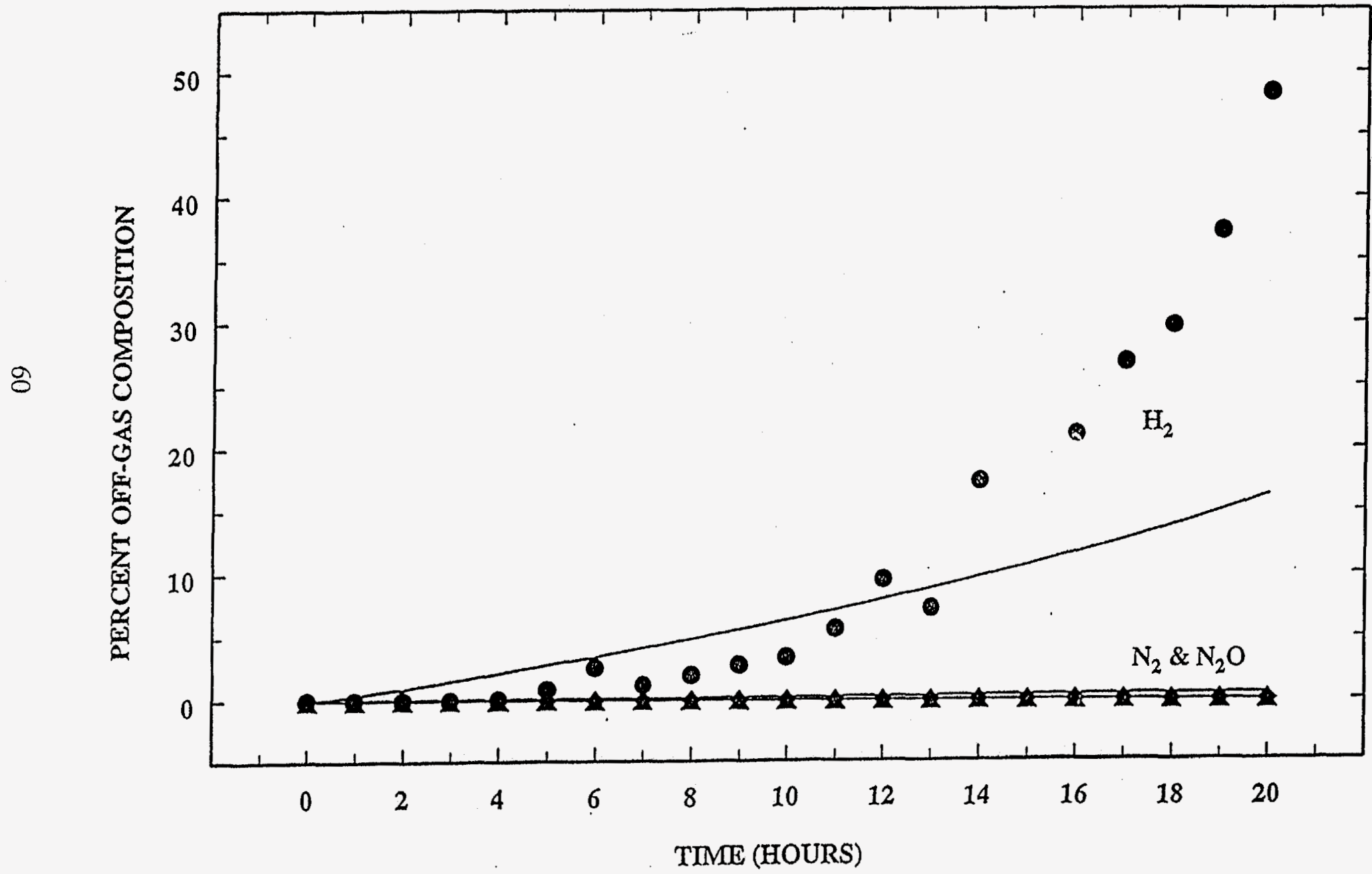


exceptional agreement which verifies the accuracy of the statistically determined exchange current densities and transfer coefficients, the methodology for determining these parameters, and the hypothesis of utilizing a boundary-layer model to extract kinetic parameters. Additional information gained from the model predictions include the moles of water transported across the membrane per mole of $\mathrm{Na}^{+}(\lambda)$ which ranged from 10.0 for experiment runs 2 and 3 to 8.2 for the full simulant run \#38. The lower value of the water transport (i.e., $\lambda=8.2$ ) for the latter run was likely due to temperature control of this experiment which was not used in experiment runs 2 and 3 . Furthermore, $\lambda$ was assumed constant for the duration of all the full simulant runs. This may not be a valid assumption with changing temperature and current density and with the life of the membrane. The relationship between the moles of water transported $(\lambda)$ and the MacMullin number $\left(N_{M}\right)$ is not completely understood at this point in the project.

\subsection{Notation}
$a, b$
coefficients defined by equation (A4)
$a_{k}{ }^{\theta}$
activity coefficient of species $k$ at standard conditions
A
projected area of the electrodes, $\mathrm{cm}^{2}$
$\mathrm{C}_{\mathrm{k}}, \mathrm{C}_{\mathrm{k}, \mathrm{a}}, \mathrm{C}_{\mathrm{k}, \mathrm{b}}$
concentration of species $\mathrm{k}$ at the electrode surface, moles $/ \mathrm{cm}^{3}$
$\mathrm{C}_{\mathrm{kb}}, \mathrm{C}_{\mathrm{k}, \mathrm{b} a}, \mathrm{C}_{\mathrm{k}, \mathrm{bc}}$
bulk concentration of species $\mathrm{k}$, moles $/ \mathrm{cm}^{3}$
$\mathrm{C}_{\mathrm{k}, \mathrm{bl}}$
$\mathrm{C}_{\text {kin }}$
$\mathrm{C}_{\text {kout }}$
$\mathrm{C}_{\mathrm{kref}}$
$\mathrm{C}_{\mathrm{H} 2 \mathrm{O}}$
concentration of species $\mathrm{k}$ at the boundary layer interface, moles $/ \mathrm{cm}^{3}$
concentration of species $\mathrm{k}$ coming into the batch reactor, moles $/ \mathrm{cm}^{3}$
concentration of species $\mathrm{k}$ leaving the batch reactor, moles $/ \mathrm{cm}^{3}$
reference concentration of species $\mathrm{k}$, moles $/ \mathrm{cm}^{3}$
$\mathrm{C}_{\mathrm{NH} 3}$
concentration of $\mathrm{H}_{2} \mathrm{O}$, moles $/ \mathrm{cm}^{3}$
$\mathrm{C}_{\mathrm{NO} 2-}$
concentration of $\mathrm{NH}_{3}$, moles $/ \mathrm{cm}^{3}$
$\mathrm{C}_{\mathrm{Na}}$
concentration of $\mathrm{NO}_{2}$, moles $/ \mathrm{cm}^{3}$
$\mathrm{C}_{\text {naNO2 }}$
concentration of $\mathrm{Na}^{+}$, moles $/ \mathrm{cm}^{3}$
$\mathrm{C}_{\text {naNO3 }}$
concentration of $\mathrm{NaNO}_{2}$, moles $/ \mathrm{cm}^{3}$
$\mathrm{C}_{\text {naOH }}$
concentration of $\mathrm{NaNO}_{3}$, moles $/ \mathrm{cm}^{3}$
concentration of $\mathrm{NaOH}$, moles $/ \mathrm{cm}^{3}$
$\mathrm{C}_{\mathrm{OH} \text { - }}$
concentration of $\mathrm{OH}^{-}$, moles $/ \mathrm{cm}^{3}$
$\mathrm{C}_{\mathrm{p}}$
specific heat at constant pressure, $\mathrm{J} / \mathrm{kg} \mathrm{K}$
$\mathrm{C}_{\text {pgas }}$
specific heat of the off gas at constant pressure, $\mathrm{J} / \mathrm{kg} \mathrm{K}$
$d_{e}$
hydraulic diameter of the electrochemical cell, $\mathrm{cm}$
$D_{k}$
diffusion coefficient of species $\mathrm{k}, \mathrm{cm}^{2} / \mathrm{sec}$ 


\begin{tabular}{|c|c|}
\hline $\mathrm{E}_{\mathrm{c}}$ & mixed potential at the cathode, $\mathrm{V}$ \\
\hline $\mathrm{E}_{\mathrm{p}}$ & energy output of centrifugal pumps, W \\
\hline F & Faraday's Constant $(96,487 \mathrm{C} / \mathrm{eq})$ \\
\hline$F_{\text {in }}$ & volumetric flow rate of gas into the batch reactor, $\mathrm{cm}^{3} / \mathrm{sec}$ \\
\hline $\mathrm{F}_{\mathrm{j}, 1}$ obs & $\begin{array}{l}\text { experimentally observed molar off-gas flow rate from reaction } \mathrm{j} \text { at time- } \\
\text { step } 1 \text {, moles/sec }\end{array}$ \\
\hline $\mathrm{F}_{\mathrm{j}, 1}$ pred & $\begin{array}{l}\text { numerically predicted molar off-gas flow rate from reaction } \mathrm{j} \text { at time-step } \\
1, \mathrm{moles} / \mathrm{sec}\end{array}$ \\
\hline $\mathrm{F}_{\mathrm{N} 2}$ & molar flow rate of $\mathrm{N}_{2}$ off-gas, moles/sec \\
\hline $\mathrm{F}_{\mathrm{N} 2 \mathrm{O}}$ & molar flow rate of $\mathrm{N}_{2} \mathrm{O}$ off-gas, moles/sec \\
\hline $\mathrm{F}_{\text {out }}$ & volumetric flow rate of gas out of the batch reactor, $\mathrm{cm}^{3} / \mathrm{sec}$ \\
\hline $\mathrm{H}_{\mathrm{k}}$ & Henry's Law Constant, mm Hg \\
\hline $\mathrm{H}_{\mathrm{p}}$ & energy input into the process due to the centrifugal pumps, W \\
\hline$i, i_{t}$ & current density through the cell defined by equation (19), $\mathrm{A} / \mathrm{cm}^{2}$ \\
\hline $\mathbf{i}_{j}, \mathbf{i}_{\mathrm{cj}}$ & partial current density of reaction $\mathrm{j}, \mathrm{A} / \mathrm{cm}^{2}$ \\
\hline $\mathrm{i}_{\mathrm{oj}, \mathrm{ref}} \mathrm{i}_{\mathrm{oj}}$ & exchange current denstiy of reation $\mathrm{j}, \mathrm{A} / \mathrm{cm}^{2}$ \\
\hline $\mathrm{K}_{\mathrm{znk}}$ & mass transfer coefficient of species $\mathrm{k}, \mathrm{cm} / \mathrm{sec}$ \\
\hline $\mathrm{L}$ & electrode length, $\mathrm{cm}$ \\
\hline $\mathrm{M}_{\mathrm{w}}$ & molecular weight of water, $\mathrm{g} / \mathrm{mole}$ \\
\hline$N_{\text {gas }}$ & total moles of gas in the reservior \\
\hline $\mathrm{N}_{\mathrm{k}}$ & molar flux of species $\mathrm{k}$, moles $/ \mathrm{cm} \mathrm{sec}$ \\
\hline $\mathrm{N}_{\mathrm{M}}$ & MacMullin number defined by equation (23) \\
\hline $\mathrm{N}_{\mathrm{Mgac}}$ & MacMullin number of the anolyte and catholyte turbulence promoters \\
\hline $\mathrm{N}_{\mathrm{Na}+}$ & molar flux of $\mathrm{Na}+$ across the membrane, moles $/ \mathrm{cm} \mathrm{sec}$ \\
\hline $\mathrm{N}_{\mathrm{w}}$ & molar flux of water across the membrane, moles $/ \mathrm{cm} \mathrm{sec}$ \\
\hline $\mathrm{Nu}_{\mathrm{k}}$ & average Nusselt number defined by equation (11) \\
\hline $\mathbf{n}_{\mathbf{j}}$ & number of electrons transferred in reaction $j$ \\
\hline $\mathbf{P}$ & pressure, $\mathrm{mm} \mathrm{Hg}$ \\
\hline $\mathbf{P}_{\mathbf{k j}}$ & reaction order for anodic reactants \\
\hline $\mathbf{P}_{\mathrm{O} 2}$ & partial pressure of $\mathrm{O}_{2}, \mathrm{~mm} \mathrm{Hg}$ \\
\hline Q & molar flow rate, moles/sec \\
\hline$Q_{\text {in }}$ & volumetric flow rate coming into the batch reactor, $\mathrm{cm}^{3} / \mathrm{s}$ \\
\hline
\end{tabular}




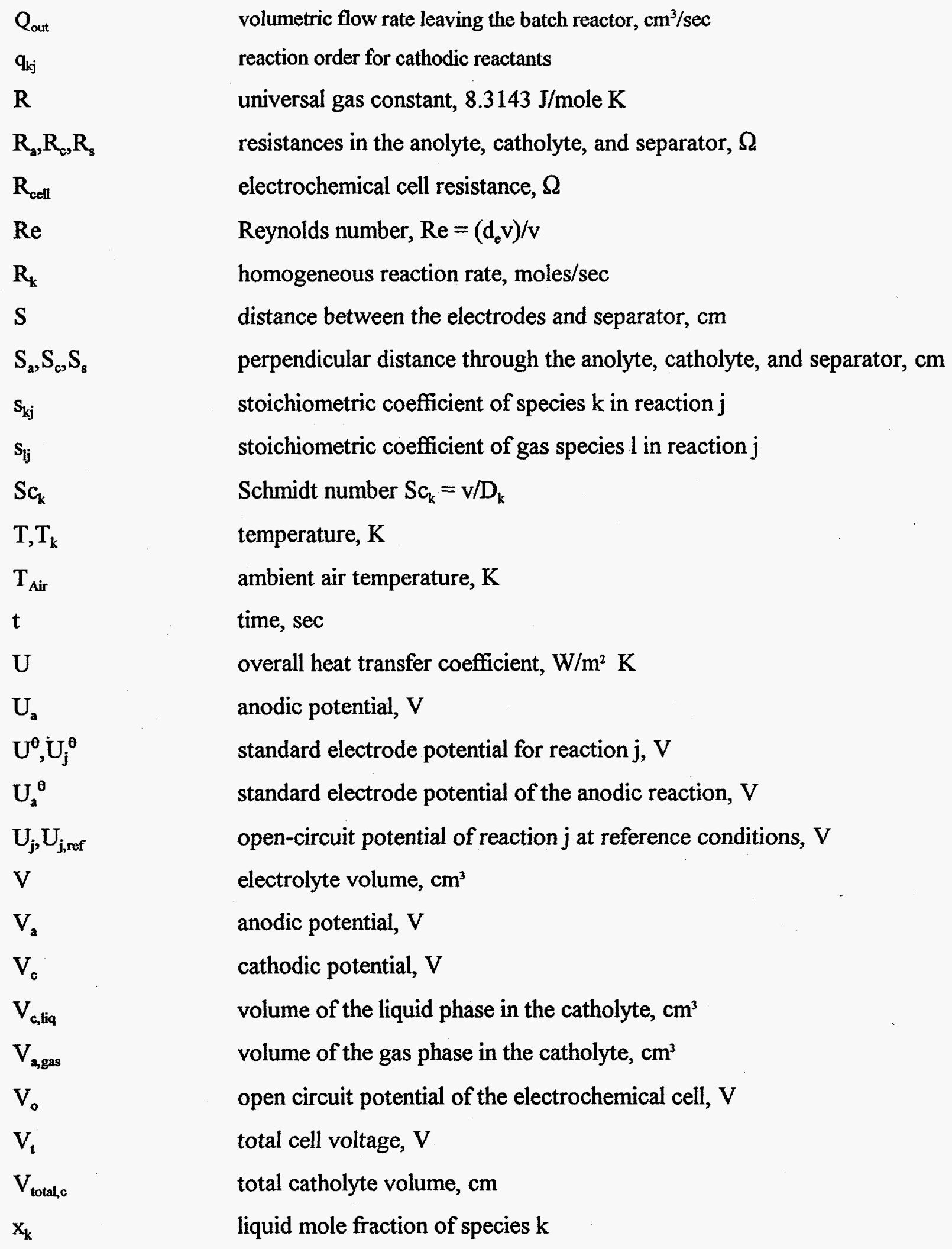

$Q_{\text {out }}$

$q_{k j}$

$R$

$\mathbf{R}_{a}, \mathbf{R}_{\iota}, \mathbf{R}_{\mathrm{s}}$

$\mathbf{R}_{\text {cell }}$

$\operatorname{Re}$

$\mathbf{R}_{\mathrm{k}}$

$S$

$\mathrm{S}_{\mathrm{a}}, \mathrm{S}_{\mathrm{c}}, \mathrm{S}_{\mathrm{s}}$

$s_{k j}$

$s_{i j}$

$S c_{k}$

$T, T_{k}$

$\mathrm{T}_{\text {Air }}$

$\mathrm{t}$

U

$\mathrm{U}_{\mathrm{a}}$

$\mathbf{U}^{\theta}, \mathbf{U}_{j}^{\theta}$

$\mathrm{U}_{\mathrm{a}}{ }^{\mathrm{\theta}}$

$\mathrm{U}_{\mathrm{j}}, \mathrm{U}_{\mathrm{j}, \text { ref }}$

$\mathrm{V}$

$\mathrm{V}_{\mathrm{a}}$

$\mathrm{V}_{\mathrm{o}}$

$\mathrm{V}_{\mathrm{c}, \mathrm{iq}}$

$\mathrm{V}_{\text {agas }}$

$\mathrm{V}$ 。

$V_{1}$

$V_{\text {totalc }}$

$\mathrm{x}_{\mathrm{k}}$

volumetric flow rate leaving the batch reactor, $\mathrm{cm}^{3} / \mathrm{sec}$

reaction order for cathodic reactants

universal gas constant, $8.3143 \mathrm{~J} / \mathrm{mole} \mathrm{K}$

resistances in the anolyte, catholyte, and separator, $\Omega$

electrochemical cell resistance, $\Omega$

Reynolds number, $\operatorname{Re}=\left(\mathrm{d}_{\mathrm{e}} \mathrm{v}\right) / \mathrm{v}$

homogeneous reaction rate, moles/sec

distance between the electrodes and separator, $\mathrm{cm}$

perpendicular distance through the anolyte, catholyte, and separator, $\mathrm{cm}$

stoichiometric coefficient of species $k$ in reaction $j$

stoichiometric coefficient of gas species $l$ in reaction $j$

Schmidt number $\mathrm{Sc}_{\mathrm{k}}=\mathrm{v} / \mathrm{D}_{\mathrm{k}}$

temperature, $\mathrm{K}$

ambient air temperature, $\mathrm{K}$

time, sec

overall heat transfer coefficient, $\mathrm{W} / \mathrm{m}^{2} \mathrm{~K}$

anodic potential, $\mathrm{V}$

standard electrode potential for reaction $\mathrm{j}, \mathrm{V}$

standard electrode potential of the anodic reaction, $\mathrm{V}$

open-circuit potential of reaction $\mathrm{j}$ at reference conditions, $\mathrm{V}$

electrolyte volume, $\mathrm{cm}^{3}$

anodic potential, $\mathrm{V}$

cathodic potential, $\mathrm{V}$

volume of the liquid phase in the catholyte, $\mathrm{cm}^{3}$

volume of the gas phase in the catholyte, $\mathrm{cm}^{3}$

open circuit potential of the electrochemical cell, $\mathrm{V}$

total cell voltage, $\mathrm{V}$

total catholyte volume, $\mathrm{cm}$

liquid mole fraction of species $\mathrm{k}$ 


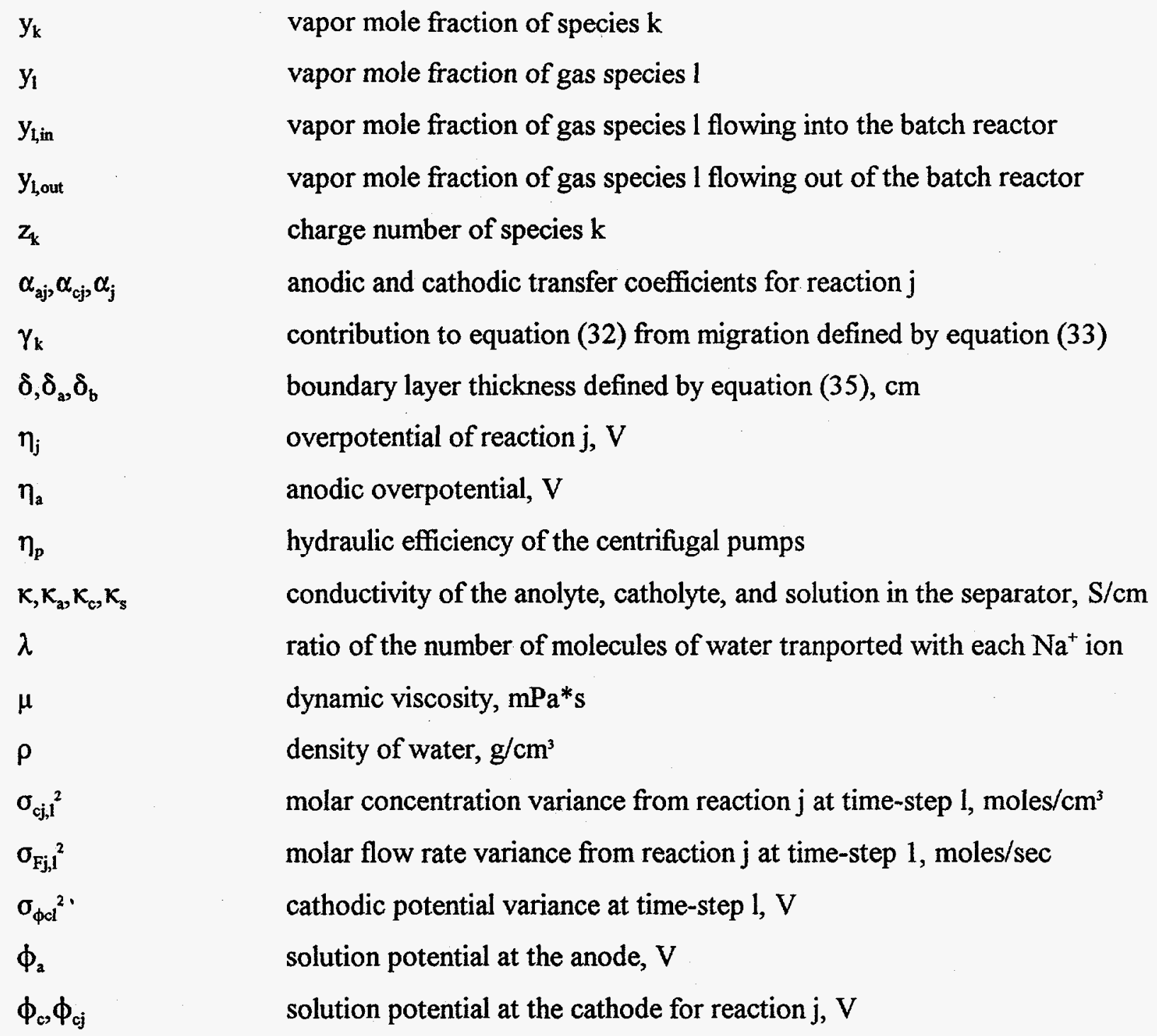

\subsection{References}

[1] D.T. Hobbs, Electrochemistry for a Cleaner Environment, J.D. Genders and N.L. Weinburg, Editors, The Electrosynthesis Company, Amherst, New York (1992).

[2] D.T. Hobbs and M. Ebra, AICHE Symp. Series No. 254, 83, 149 (1987).

[3] J.D. Genders, N.L. Weinberg, and D. Hartsough, by Electrosynthesis Inc. Phase II Report, by Electrosynthesis Inc.WSRC TR 90 491(1992)

[4] D.H. Coleman and R.E. White, J. Electrochemical Society, 142, 1152 (1995).

[5] S. Prasad, J.W. Weidner, and A.E. Farell, J. Electrochemical Society, 142, 3815 (1995).

[6] J.A. Cox and A. Brajter, Electrochim. Acta, 24, 517 (1979).

[7] D. Pletcher and Z. Poorabedi, Electrochim. Acta, 24, 1253 (1979). 
[8] X. Xing, D.A. Scherson, and C. Mak, J. Electrochemical Society, 137, 2166 (1990).

[9] K.E. Johnson and D.T. Sawyer, J. Electroanal. Chem., 49, 95 (1974).

[10] G. Horanyi and E.M. Rizmayer, J. Electroanal. Chem., 188, 265 (1985).

[11] H.L. Li, D.H. Robertson, J.Q. Chambers, and D.T. Hobbs, J. Electrochemical Society, 135, 1154 (1988).

[12] H.L. Li, J.Q. Chambers, and D.T. Hobbs, J. Appl. Electrochem, 18, 454 (1988).

[13] H.L. Li, W.C. Anderson, J.Q. Chambers, and D.T. Hobbs, Inorg. Chem., 28, 863 (1989).

[14] S. Cattarin, J. Appl. Electrochem., 22, 1077 (1992).

[15] D.A. Wingard, Ph.D Dissertation, Dept. Of Chemical Engineering, University of South Carolina (1996).

[16] M. J. Mader, C. W. Walton, and R. E. White, J. Electrochemical Society, 133 (1986) 1124.

[17] R. B. Bird, W. E. Steward, and E. N. Lightfoot, Transport Phenomena, John Wiley \& Sons, New York, NY, (1960).

[18] J. S. Newman, Ind. Eng. Chem. Fundam, 5 (1966) 525.

[19] J. S. Newman, 'Electrochemical Systems,' 2nd ed., Prentice Hall, Englewood Cliffs, NJ (1991).

[20] R. Caban and T. W. Chapman, Chem. Eng. Sci., 36 (1981) 849.

[21] R. D. Engelken and T. P. Van Doren, J. Electrochemical Society, 132 (1985) 2904.

[22] F. Lapicque and A. Stork, J. Appl. Electrochem., 15 (1985) 925.

[23] V. Edwards and J. S. Newman, J. Electrochemical Society, 134 (1987) 1181.

[24] G. D. Simpson and R. E. White, J. Electrochemical Society, 136 (1989) 2137.

[25] J. C. Smeltzer and P. S. Fedkiw, J. Electrochemical Society, 139 (1992) 1358.

[26] J. C. Smeltzer and P. S. Fedkiw, J. Electrochemical Society, 139 (1992) 1366.

[27] D. J. Picket, Electrochim. Acta, 18 (1973) 835.

[28] A. T. S. Walker and A. A. Wragg, Electrochim. Acta, 22 (1977) 1129.

[29] K. Scott, Electrochim. Acta, 30 (1985) 235.

[30] K. Scott, Electrochim. Acta, 30 (1985) 245.

[31] K. Scott, J. Appl. Electrochem., 15 (1985) 837.

[32] K. Scott, J. Appl. Electrochem., 15 (1985) 859.

[33] T. V. Nguyen, C. W. Walton, and R. E. White, J. Electrochemical Society, 133 1986) 1130.

[34] L. Weise, G. Valentin, A. Stork, R. Mauge, and A. Cohen, J. Appl. Electrochem., 16 (1986) 836.

[35] G. D. Simpson and R. E. White, J. Electrochemical Society, 137 (1990) 1843.

[36] R. Bakshi and P. S. Fedkiw, J. Appl. Electrochem., 23 (1993) 715.

[37] K. Scott and E. M. Payton, Electrochim. Acta, 38 (1993) 2181.

[38] S. I. Sandler, Chemical and Engineering Thermodynamics, 2nd ed., John Wiley \& Sons, New York, NY (1989).

[39] A. J. Bard and L. R. Faulkner, Electrochemical Methods, John Wiley \& Sons, New York, NY (1980).

[40] R. E. White, J. S. Beckerdite, and J. W. Van Zee, 'Electrochemical Cell Design,' R. 
E. White, Editor, Plenum Press, NY (1984).

[41] S. M. Walas, Chemical Process Equipment, Butterworth-Heineman, Stoneham, MA (1990).

[42] W. Grot, personal communication, E. I. duPont de Nemours \& Co., Nafion Products, Wilmington, DE 19898 (1994).

[43] IMSL Math/Library, Problem-Solving Software Systems, Edition 1.1., Houston, TX 77042 (1989).

[44] D. Coleman, M.S. thesis, Texas A\&M University, College Station , TX (1993).

[45] D. S. Moore and G. P. McCabe, Introduction to the Practice of Statistics, W. H. Freeman and Co., New York, NY (1989).

[46] J. Neter, W. Wasserman, and M. H. Kutner, Applied Linear Statistical Models, 3rd ed., Irwin, Burr Ridge, IL (1990).

[47] J. W. Van Zee, Ph.D. dissertation, Texas A\&M University, Houston, TX (1984). 


\subsection{Appendices}

\subsection{Appendix A}

Physical Properties Data Analysis

Density $(\rho)$, viscosity $(\eta)$, heat capacities $\left(C_{\rho}\right)$, and conductivity $(\kappa)$ data for the system of sodium hydroxide, sodium nitrate, and sodium nitrite in water from 30 to $85^{\circ} \mathrm{C}$ is presented in Appendix A. Empirical correlations were developed for these properties as a function of temperature and concentration. This work considered a limited range of concentrations and temperatures of interest in modeling the electrochemical reduction process. No literature references were found for aqueous ternary salt solutions and relatively little on binary aqueous salt solutions.

Empirical mixing rules have been derived for the viscosity of dilute aqueous binary solutions based on their single salt behavior that agree with $\pm 2 \%$. These mixing rules did not accurately model data obtained in this work and, thus, regression models were developed that are accurate to within $\pm 10 \%$ for concentrated solutions used in this work. Physical property data for the aqueous single salt systems of sodium hydroxide, sodium nitrate, and sodium nitrite were used to verify experimental methods.

\section{Materials}

ACS Grade sodium nitrate and sodium nitrite were used and weighed directly. ACS Grade sodium hydroxide pellets were stored in a desiccator and prior to use. Deionized water (18 $\mathrm{M} \Omega$ ) was obtained from a Millipore wall mount system and used for all solutions. Stock solutions of sodium hydroxide, sodium nitrate and sodium nitrite were prepared to eliminate any variations in weighing. The final concentrations of the sodium hydroxide stock solution were obtained from the average of three titrations with $99.9 \%$ acetic acid. All solutions were prepared by titrating the necessary volume from these stock solutions and then diluting to volume. The error in the molar concentrations is estimated to be $\pm 0.003 \mathrm{~mol} / \mathrm{L}$. This was verified by analyzing random samples by ion chromatography with the estimated concentrations agreeing well within the instrumental error.

\section{Measurements}

Measurements were performed using a partial $3^{3}$ factorial design of the predicted solution compositions ranging from $1.33-6.0 \mathrm{M}$ sodium hydroxide, $0.1-1.95 \mathrm{M}$ sodium nitrate, and 0.03 - $1.9 \mathrm{M}$ sodium nitrate. These measurements were obtained at $30,45,60$, and $85^{\circ} \mathrm{C}$. Densities $\rho$ of the solutions were measured using a PAAR DMA 48 density meter. Standard procedures were followed using deionized water and $4 \mathrm{M} \mathrm{NaOH}$ solution for calibration at the temperatures of interest. This instrument has a specified accuracy of $\pm 1.0 \times 10^{-4} \mathrm{~g} / \mathrm{cm}^{3}$. An average of two measurements is reported, and these were reproducible within $\pm 0.0001 \mathrm{~g} / \mathrm{cm}^{3}$. Densities at $85^{\circ} \mathrm{C}$ were not obtained due to this temperature being outside of the instrument's operating range. The precision of the temperature control was $\pm 0.1 \mathrm{C}$.

Viscosities $\eta$ of the solutions were measured using a Brookfield digital viscometer Model DV-II with a CP-40 cone. Standard procedures were followed and verified the calibration by using standards by Brookfield with viscosities of 1.0 and $3.0 \mathrm{mPa} \mathrm{s}$ at $30^{\circ} \mathrm{C}$. An average of two measurements is reported, and these were reproducible within $\pm 0.02 \mathrm{mPa}-\mathrm{s}$.

Heat capacities $\left(C_{\rho}\right)$ of the solutions were measured using a Perkin-Elmer DSC7 with a 
temperature control bath attached. Comparison of the obtained measurements with International Critical Tables literature values of the $\mathrm{NaOH} / \mathrm{H}_{2} \mathrm{O}$ systems indicated that corrections to heat capacities were unnecessary. A scanning rate of 3 degrees/minute was determined to be the most accurate from the determinations of the heat capacity of deionized water. An average of three measurements is reported, and these were reproducible within $\pm 0.0002 \mathrm{~J} / \mathrm{g}^{\circ} \mathrm{C}$.

Conductivities $(\mathrm{K})$ of these were measured using a YSI Model 34 conductivity meter with a YSI 3417 Conductivity Cell. Data at the temperatures of interest were obtained by immersion in a controlled temperature bath of the flask containing the solutions. The temperatures were verified with a calibrated thermometer. The conductivity probes were verified by checking pure sodium hydroxide solutions against their literature values. An average of three measurements is reported, and these were reproducible within \pm 0.01 Siemens $/ \mathrm{cm}$. This error is believed high due to fouling of the cell even upon cleaning with a boric acid rinse.

\section{Empirical Correlations}

The experimental results of $\rho, \eta, C_{\rho}$, and $\kappa$ of the three sodium hydroxide solutions and the thirteen ternary mixtures at the four temperatures are given in Table A-1. The dependency density and conductivity on concentration and temperature for the solutions were found to be linear:

$$
\begin{aligned}
\rho\left(T_{K}, C_{\mathrm{NaOH}}, C_{\mathrm{NaNO}_{3}} C_{\left.\mathrm{NaNO}_{2}\right)=}(0.03939) C_{\mathrm{NaOH}}+(0.05755) C_{\mathrm{NaNO}_{3}}\right. \\
+(0.04344) C_{\mathrm{NaNO}_{2}}-(0.00126) T_{K} \\
+1.47258
\end{aligned}
$$

$$
\begin{aligned}
& \kappa\left(\mathrm{T}_{\mathrm{K}}, \mathrm{C}_{\mathrm{NaOH}}, \mathrm{C}_{\mathrm{NaNO}_{3}}, \mathrm{C}_{\mathrm{NaNO}_{2}}\right)=\left(C_{\mathrm{NaOH}}+C_{\mathrm{NaNO}_{3}}+C_{\mathrm{NaNO}_{2}}\right)\left((0.06833) \sqrt{C_{\mathrm{NaOH}} \mathrm{CNaNO}_{3}}\right.
\end{aligned}
$$

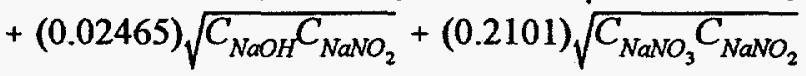

$$
\begin{aligned}
& \text { - }(0.0006447) T_{K} \sqrt{C_{\mathrm{NaOH}}}+(0.0008327) T_{K} \sqrt{C_{\mathrm{NaNO}_{3}}} \\
& +(0.0004489) T_{K} \sqrt{C_{\mathrm{NaNO}_{2}}}+(0.08448) \sqrt{C_{\mathrm{NaOH}}} \\
& +(0.09601) \sqrt{C_{\mathrm{NaNO}_{3}}}+(0.04501) \sqrt{C_{\mathrm{NaNO}_{2}}}
\end{aligned}
$$


The density correlation is a simple linear fit of the data and its inherent error is presented in Figure A-1. The conductivity correlation is based on the Kohlrausch Equation and includes binary interaction terms to obtain a fit with the desired accuracy: Figure A-2 shows the validity of this equation. A study was performed on the data with the equation to determine the validity of each individual term and elimination of any single term that introduced unacceptable error for application. All of the terms shown in equations Al through A4 are required.

The viscosity correlation was found to be a linear function of concentration and to have an exponential dependence on temperature:

$$
\begin{array}{r}
\left(\mu\left(T_{K}, C_{\mathrm{NaOH}}, C_{\mathrm{NaNO}_{3}}, C_{\mathrm{NaNO}_{2}}\right)\right)=(0.3129) C_{\mathrm{NaOH}}+(0.1607) C_{\mathrm{Na}} \\
+(0.1521) C_{\mathrm{NaNO}_{2}}-7.920+\frac{(228}{T_{K}}
\end{array}
$$

Figure A-3 illustrates the accuracy of the correlation in the prediction of the viscosity. Relative percent errors for correlations (A-1 - A-3) are presented in Table A-2. below:

Heat capacities were found fit the standard equation for temperature dependence indicated

$$
C_{P}=a+b * T+\frac{c}{T^{2}}
$$

The coefficients for this equation and the solutions studied are presented in Table A-3. However, there are not enough data points to determine any concentration dependencies.

The study of this system allows more precise modeling of the electrochemical processes that can be applied to assist in the treatment of waste streams containing large nitrate/nitrite concentrations and the increase of caustic concentration associated with their destruction. This data also allows temperature and heating effects to be likewise modeled. 
Table A1. Summary of Physical Properties

\begin{tabular}{|c|c|c|c|c|c|c|c|c|c|c|c|c|c|c|c|c|c|}
\hline \multirow{2}{*}{$\begin{array}{l}\mathrm{C} \\
\mathrm{NaOH}) \\
\mathrm{mol} / \mathrm{L}\end{array}$} & \multirow{2}{*}{$\begin{array}{l}\mathrm{C} \\
\left(\mathrm{NaNO}_{3}\right) \\
\mathrm{mol} / \mathrm{L}\end{array}$} & \multirow{2}{*}{$\begin{array}{l}\mathrm{C} \\
\left(\mathrm{NaNO}_{3}\right) \\
\mathrm{mol} / \mathrm{L}\end{array}$} & \multicolumn{4}{|c|}{$30^{\circ} \mathrm{C}$} & \multicolumn{4}{|c|}{$45^{\circ} \mathrm{C}$} & \multicolumn{4}{|c|}{$60^{\circ} \mathrm{C}$} & \multicolumn{3}{|c|}{$85^{\circ} \mathrm{C}$} \\
\hline & & & $\begin{array}{l}\rho \\
\mathrm{g} / \mathrm{ml}\end{array}$ & $\begin{array}{l}\eta \\
\mathrm{mPa}^{*}{ }^{*} \mathrm{~s}\end{array}$ & $\begin{array}{l}\kappa \\
1 /(\Omega * \mathrm{~cm})\end{array}$ & $\begin{array}{l}C_{P} \\
J /\left(g^{*} \mathrm{~K}\right)\end{array}$ & $\begin{array}{l}\rho \\
g / m l\end{array}$ & $\prod_{\mathrm{mPa}^{*} \mathrm{~s}_{\mathrm{s}}}$ & $\begin{array}{l}\kappa \\
1 /\left(\Omega *^{*} \mathrm{~cm}\right)\end{array}$ & $\begin{array}{l}\mathrm{C}_{\mathrm{P}} \\
\mathrm{J} /\left(\mathrm{g}^{*} \mathrm{~K}\right)\end{array}$ & $\begin{array}{l}\rho \\
\mathrm{g} / \mathrm{ml}\end{array}$ & $\underset{\mathrm{mPa}^{*} \mathrm{~s}}{\eta}$ & $\begin{array}{l}\kappa \\
1 /(\Omega * \mathrm{~cm})\end{array}$ & $\begin{array}{l}\mathrm{C}_{\mathrm{P}} \\
\mathrm{J} /\left(\mathrm{g}^{*} \mathrm{~K}\right)\end{array}$ & $\prod_{\mathrm{mPa}^{*} \mathrm{~s}}$ & $\begin{array}{l}\kappa \\
1 /(\Omega * \mathrm{~cm})\end{array}$ & $\mathrm{J}_{\mathrm{J} /\left(\mathrm{g}^{*} \mathrm{~K}\right)}$ \\
\hline \multirow[t]{5}{*}{1.33} & 0.00 & 0.00 & 1.0924 & 1.11 & 0.253 & 3.8214 & 1.0744 & 0.77 & 0.318 & 3.8842 & 1.0572 & 0.53 & 0.398 & 3.9199 & 0.27 & 0.494 & 3.9624 \\
\hline & 1.95 & 0.60 & 1.2991 & 1.46 & 0.261 & 3.1439 & 1.2798 & 1.02 & 0.341 & 3.1111 & 1.2597 & 0.80 & 0.398 & 3.0760 & 0.49 & 0.503 & 3.0103 \\
\hline & & 11.90 & 1.3675 & 1.83 & 0.221 & 3.0174 & 1.3475 & 1.31 & 0.336 & 3.0244 & 1.3270 & 0.96 & 0.371 & 3.0034 & 0.55 & 0.501 & 2.9612 \\
\hline & 0.75 & 0.60 & 1.2195 & 1.29 & 0.260 & 3.1517 & 1.2014 & 0.92 & 0.371 & 3.1520 & 1.1824 & 0.64 & 0.400 & 3.2735 & 0.32 & 0.498 & 3.1858 \\
\hline & & 0.75 & 1.2619 & 1.61 & 0.270 & 3.2359 & 1.2430 & 1.17 & 0.352 & 3.2142 & 1.2233 & 0.82 & 0.428 & 3.1558 & 0.48 & 0.561 & 3.1098 \\
\hline \multirow[t]{6}{*}{3.00} & 0.00 & 0.00 & 1.2322 & 1.67 & 0.345 & 3.4016 & 1.2146 & 1.19 & 0.475 & 3.4358 & 1.1962 & 0.89 & 0.577 & 3.4443 & 0.50 & 0.728 & 3.4542 \\
\hline & 1.95 & 1.90 & 1.3684 & 3.12 & 0.282 & 3.1519 & 1.3490 & 2.07 & 0.389 & 3.1440 & 1.3289 & 1.56 & 0.475 & 3.1061 & 0.99 & 0.646 & 3.0824 \\
\hline & & 0.60 & 1.2694 & 2.50 & 0.377 & 3.2663 & 1.2513 & 1.72 & 0.402 & 3.2875 & 1.2322 & 1.22 & 0.612 & 3.2837 & 0.71 & 0.800 & 3.2788 \\
\hline & 0.75 & 1.90 & 1.3429 & 2.03 & 0.295 & 3.2422 & 1.3239 & 1.32 & 0.402 & 3.2909 & 1.3043 & 0.89 & 0.495 & 3.3119 & 0.56 & 0.662 & 3.2700 \\
\hline & & 0.03 & 1.2461 & 1.84 & 0.366 & 2.8720 & 1.2280 & 1.31 & 0.443 & 2.9206 & 1.2092 & 0.83 & 0.539 & 2.9442 & 0.53 & 0.691 & 2.9635 \\
\hline & 0.10 & 1.90 & 1.3041 & 2.15 & 0.307 & 3.3354 & 1.2856 & 1.44 & 0.425 & 3.3595 & 1.2662 & 0.99 & 0.532 & 3.3593 & 0.57 & 0.707 & 3.3026 \\
\hline \multirow[t]{5}{*}{6.00} & 0.00 & 0.00 & 1.3815 & 3.53 & 0.365 & 3.5575 & 1.3629 & 2.38 & 0.527 & 3.6005 & 1.3439 & 1.73 & 0.691 & 3.6127 & 1.01 & 0.962 & 3.5874 \\
\hline & 0.75 & 1.90 & 1.4396 & 6.22 & 0.279 & 3.2152 & 1.4204 & 3.58 & 0.414 & 3.2403 & 1.4005 & 2.46 & 0.536 & 3.2319 & 1.46 & 0.795 & 3.2407 \\
\hline & & 0.03 & 1.3504 & 4.16 & 0.290 & 3.3669 & 1.3318 & 2.76 & 0.530 & 3.4003 & 1.3126 & 1.96 & 0.566 & 3.4067 & 1.19 & 0.721 & 3.4249 \\
\hline & 0.10 & 1.90 & 1.4147 & 4.97 & 0.222 & 3.2665 & 1.3958 & 3.06 & 0.334 & 3.3038 & 1.3761 & 1.98 & 0.427 & 3.3199 & 1.04 & 0.579 & 3.3262 \\
\hline & & 0.03 & 1.3097 & 3.55 & 0.181 & $3: 4149$ & 1.2916 & 2.41 & 0.639 & 3.4873 & 1.2729 & 1.69 & 0.609 & 3.5268 & 0.93 & 0.844 & 3.5538 \\
\hline
\end{tabular}


Table A2. Relative Percent Error of Empirical Correlations Summary

\begin{tabular}{|c|c|c|c|c|c|c|c|c|c|c|c|c|c|}
\hline \multirow{2}{*}{$\begin{array}{l}\mathrm{C} \\
(\mathrm{NaOH}) \\
\mathrm{mol} / \mathrm{L}\end{array}$} & \multirow{2}{*}{$\begin{array}{l}\mathrm{C} \\
\left(\mathrm{NaNO}_{3}\right) \\
\mathrm{mol} / \mathrm{L}\end{array}$} & \multirow{2}{*}{$\begin{array}{l}\mathrm{C} \\
\left(\mathrm{NaNO}_{2}\right) \\
\mathrm{mol} / \mathrm{L}\end{array}$} & \multicolumn{4}{|c|}{$30^{\circ} \mathrm{C}$} & \multicolumn{2}{|l|}{$45^{\circ} \mathrm{C}$} & \multirow[b]{2}{*}{$\rho$} & \multicolumn{2}{|l|}{$60^{\circ} \mathrm{C}$} & \multicolumn{2}{|l|}{$85^{\circ} \mathrm{C}$} \\
\hline & & & $\rho$ & $\eta$ & $\kappa$ & $\rho$ & $\eta$ & $\kappa$ & & $\eta$ & $\kappa$ & $\eta$ & $\kappa$ \\
\hline \multirow[t]{5}{*}{1.33} & 0.00 & 0.00 & -4.63 & -1.05 & 2.19 & -4.63 & -0.40 & -0.86 & -4.54 & -2.66 & -6.53 & 12.13 & -5.55 \\
\hline & 1.95 & 0.60 & 1.37 & 18.30 & -4.29 & 1.36 & 14.00 & 5.09 & 1.29 & 1.41 & 11.03 & -2.84 & 10.21 \\
\hline & & 1.90 & 2.17 & 8.64 & -15.36 & 2.12 & 2.17 & -16.44 & 2.04 & -2.73 & -13.98 & -0.36 & -3.62 \\
\hline & 0.75 & 0.60 & 0.60 & 5.47 & 0.39 & 0.67 & -0.43 & 0.10 & 0.67 & -0.14 & -1.83 & 17.20 & -3.84 \\
\hline & & 1.90 & -0.54 & -2.73 & -2.58 & -0.55 & -9.88 & 0.24 & -0.62 & -1.03 & 3.42 & -10.06 & 5.58 \\
\hline \multirow[t]{6}{*}{3.00} & 0.00 & 0.00 & 1.90 & 0.73 & 13.23 & 2.03 & -4.83 & 5.64 & 2.11 & -11.22 & 6.24 & -7.26 & 9.66 \\
\hline & 1.95 & 1.90 & -2.56 & -2.41 & 24.08 & -2.64 & -0.97 & 8.16 & -2.77 & -8.32 & 3.49 & -15.22 & -5.63 \\
\hline & & 0.60 & -0.68 & -16.65 & -1.22 & -0.62 & -18.44 & 0.61 & -0.65 & -19.77 & -0.99 & -19.10 & 3.37 \\
\hline & 0.75 & 1.90 & 0.63 & 18.15 & 6.94 & 0.63 & 22.23 & 2.32 & 0.58 & 26.58 & 2.46 & 18.06 & 0.74 \\
\hline & & 0.03 & -0.57 & 6.47 & -4.84 & -0.52 & 0.68 & 0.02 & -0.52 & 10.87 & -0.20 & 1.89 & 0.71 \\
\hline & 0.10 & 1.90 & -0.54 & -1.97 & -1.04 & 0.58 & -1.46 & -0.48 & 0.55 & 0.00 & 1.91 & 1.92 & 4.77 \\
\hline \multirow[t]{5}{*}{6.00} & 0.00 & 0.00 & 3.95 & 2.48 & -16.72 & 4.02 & 2.34 & -8.02 & 4.07 & -1.78 & -3.69 & -1.27 & 0.49 \\
\hline & 0.75 & 1.90 & -0.91 & -17.07 & 33.70 & -0.94 & -3.00 & 11.05 & -1.02 & -1.51 & 1.95 & -2.62 & 13.08 \\
\hline & & 0.03 & -1.56 & 1.28 & -1.99 & -1.56 & 2.77 & -8.65 & -1.60 & 0.97 & -5.88 & -2.41 & -1.17 \\
\hline & 0.10 & 1.90 & -0.04 & -8.80 & -32.22 & -0.04 & -0.27 & -15.58 & -0.10 & 7.53 & -2.21 & 20.14 & 0.81 \\
\hline & & 0.03 & -1.86 & 4.29 & 21.18 & -1.82 & 3.43 & 1.08 & -1.83 & 2.90 & -2.19 & 9.73 & 1.96 \\
\hline
\end{tabular}


Table A3. Heat Capacity Correlation Coefficients

\begin{tabular}{|c|c|c|c|c|c|c|c|c|c|}
\hline \multirow{2}{*}{$\begin{array}{l}\mathrm{C} \\
(\mathrm{NaOH}) \\
\mathrm{mol} / \mathrm{L}\end{array}$} & \multirow{2}{*}{$\begin{array}{l}\mathrm{C} \\
\left(\mathrm{NaNO}_{3}\right) \\
\mathrm{mol} / \mathrm{L}\end{array}$} & \multirow{2}{*}{$\begin{array}{l}\mathrm{C} \\
(\mathrm{NaNO} 2) \\
\mathrm{mol} / \mathrm{L}\end{array}$} & \multicolumn{4}{|c|}{ Coefficients } & \multicolumn{3}{|c|}{ Relative Percent Error at Temperature } \\
\hline & & & $\mathrm{a}$ & $\mathrm{b}$ & c & $30^{\circ} \mathrm{C}$ & $45^{\circ} \mathrm{C}$ & $60^{\circ} \mathrm{C}$ & $85^{\circ} \mathrm{C}$ \\
\hline \multirow[t]{5}{*}{1.33} & 0.00 & 0.00 & 6.623 & -0.00465 & -127800 & -0.046 & 0.069 & -0.078 & 0.012 \\
\hline & 1.95 & 0.60 & 4.576 & -0.00383 & -24990 & 0.013 & 0.001 & 0.021 & 0.012 \\
\hline & & 1.90 & 6.317 & -0.00705 & -106600 & -0.052 & 0.15 & -0.12 & 0.04 \\
\hline & 0.75 & 0.60 & 11.945 & -0.01730 & -327800 & 0.53 & -1.62 & 1.36 & -0.29 \\
\hline & & 1.90 & 3.704 & -0.00187 & 9519 & -0.11 & 0.38 & -0.31 & 0.084 \\
\hline \multirow[t]{6}{*}{3.00} & 0.00 & 0.00 & 5.696 & -0.00426 & -92090 & -0.053 & 0.12 & -0.11 & 0.024 \\
\hline & 1.95 & 1.90 & 3.520 & -0.00126 & 1654 & -0.096 & 0.31 & -0.25 & 0.065 \\
\hline & & 0.60 & 5.331 & -0.00408 & -75870 & -0.055 & 0.14 & -0.12 & 0.031 \\
\hline & 0.75 & 1.90 & 10.807 & -0.01502 & -276800 & 0.007 & -0.095 & 0.072 & -0.003 \\
\hline & & 0.03 & 5.965 & -0.00558 & -128600 & -0.049 & 0.073 & -0.079 & 0.016 \\
\hline & 0.10 & 1.90 & 9.530 & -0.01269 & -215600 & 0.001 & -0.043 & 0.038 & 0.007 \\
\hline \multirow[t]{5}{*}{6.00} & 0.00 & 0.00 & 9.254 & -0.001126 & -209900 & -0.025 & 0.019 & -0.022 & 0.014 \\
\hline & 0.75 & 1.90 & 4.781 & -0.00291 & -64140 & 0.43 & 0.56 & -0.071 & 0.042 \\
\hline & & 0.03 & 4.943 & -0.00278 & -67240 & -0.066 & 0.16 & -0.15 & 0.031 \\
\hline & 0.10 & 1.90 & 6.230 & -0.00555 & -11770 & -0.032 & 0.045 & -0.049 & 0.012 \\
\hline & & 0.03 & 8.235 & -0.00872 & -199800 & -0.045 & 0.042 & -0.057 & 0.011 \\
\hline
\end{tabular}


Figure A1 : Comparison of Predicted and Observed Density

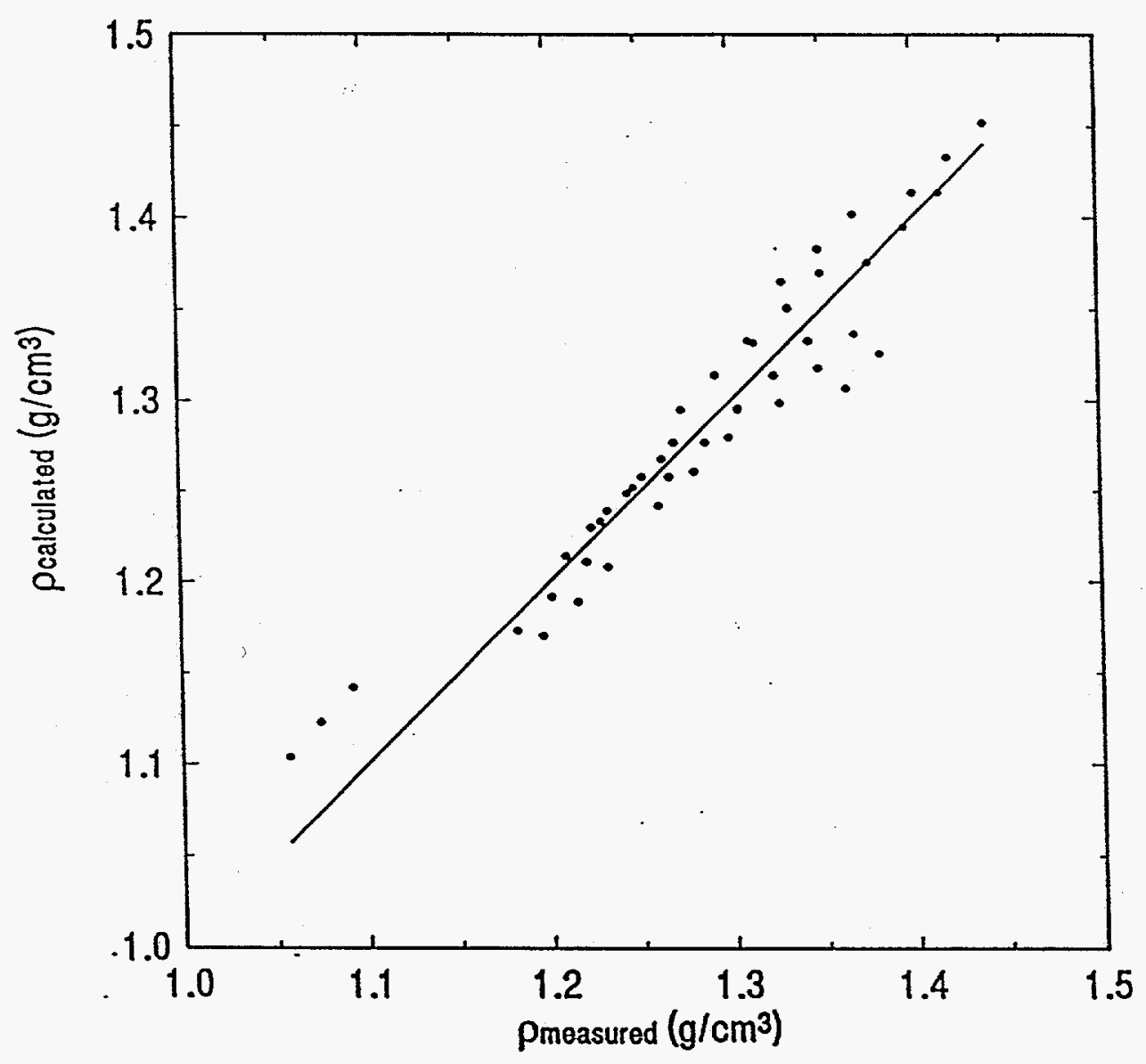


Figure A2 : Comparison of Predicted and Observed Conductivity

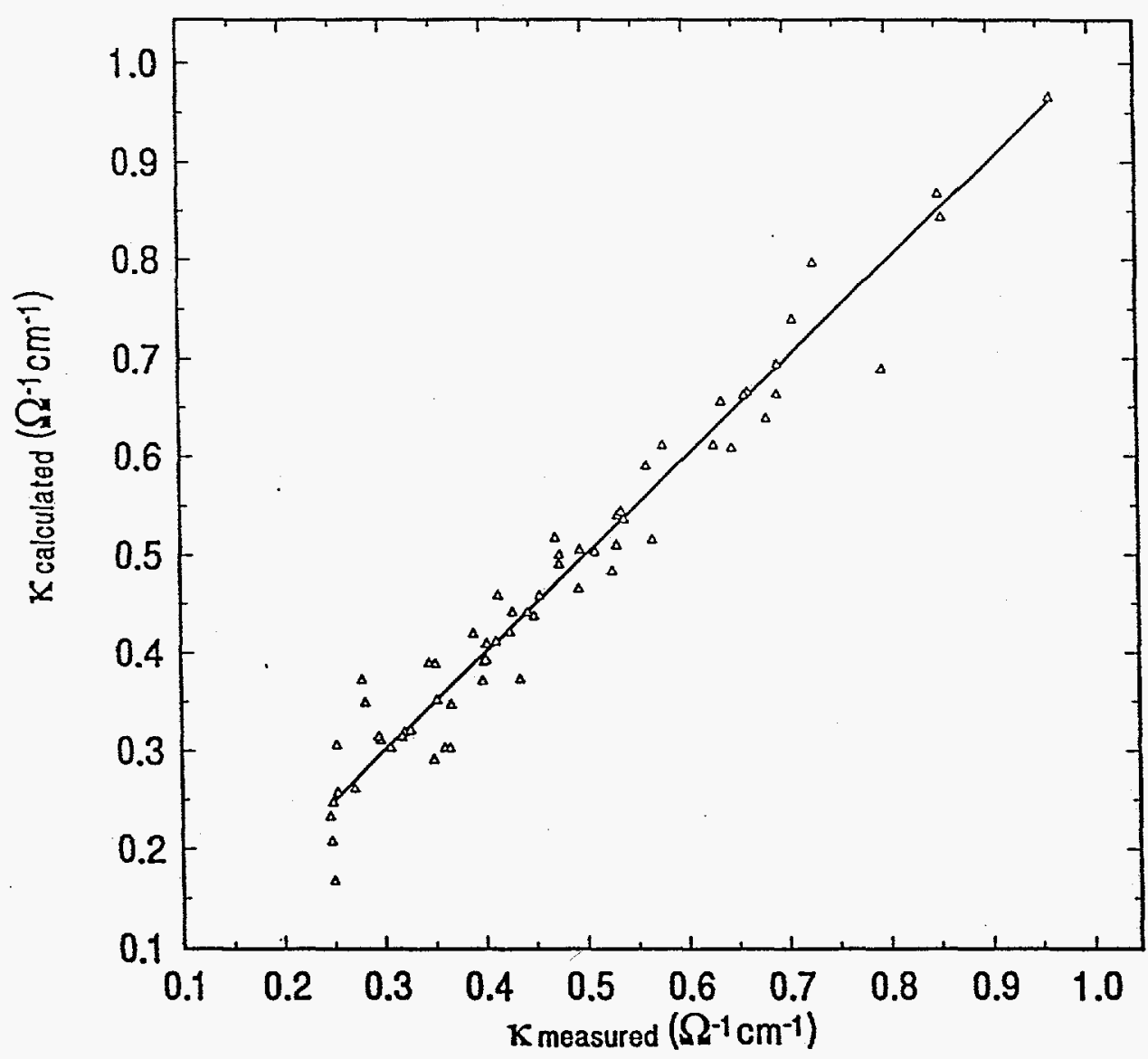


Figure A3 : Comparison of Predicted and Observed Viscosity

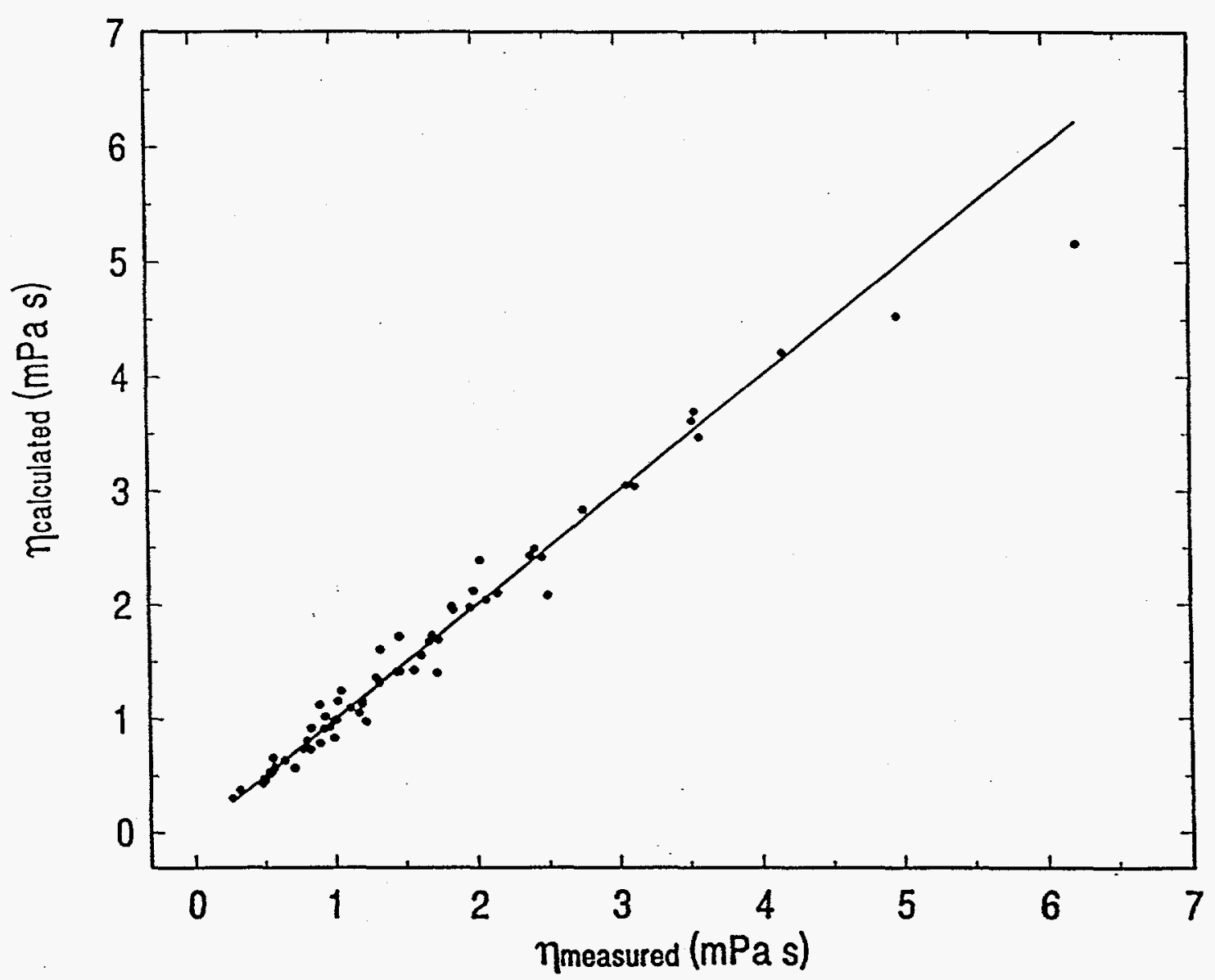




\subsection{Appendix B}

Experimental Data for Full Simulant Run

The experimental data shown in Tables B-1 and B-2 correspond to experimental runs 2 and 3. These experiments were full simulant batch runs with nominal initial concentrations in the catholyte of $1.95 \mathrm{M} \mathrm{NaNO}_{3}, 0.60 \mathrm{M} \mathrm{NaNO}_{2}$, and $1.33 \mathrm{M} \mathrm{NaOH}$, and an anolyte composed of 5.0 $\mathrm{MNaOH}$.

Table B-1. Experiment 2 Constant Current Data for the Reduction of $\mathrm{NO}_{3}^{-}, \mathrm{NO}_{2}^{-}$, and $\mathrm{H}_{2} \mathrm{O}$ at a Nickel Cathode and the Oxidation of $\mathrm{OH}^{-}$at a Stainless Steel Anode.

\begin{tabular}{|c|c|c|c|c|c|}
\hline \multicolumn{4}{|c|}{ Electrode Materials: Nickel Cathode, Stainless Steel Anode } & \multicolumn{2}{|c|}{ Date:07/21/94-07/26/94 } \\
\hline \multicolumn{6}{|c|}{ Current Density: $0.25 \mathrm{~A} / \mathrm{cm}_{2}$} \\
\hline Electrolysis & Measured & Catholyte & Catholyte & Catholyte & Anolyte \\
\hline Time (Hours) & $\begin{array}{c}\text { Anode vs } \\
\text { Cathode(V) }\end{array}$ & $\begin{array}{c}\mathrm{NO}_{3}^{-} \text {Conc. } \\
\text { (M) }\end{array}$ & $\begin{array}{c}\mathrm{NO}_{2}^{-} \text {Conc. } \\
\text { (M) }\end{array}$ & $\begin{array}{l}\mathrm{OH}^{-} \text {Conc. } \\
\text { (M) }\end{array}$ & Conc. (M) \\
\hline 0 & 4.88 & 2.01 & 0.60 & 1.40 & 5.71 \\
\hline 1.5 & 4.48 & 1.91 & 0.53 & 1.72 & 5.52 \\
\hline 3.0 & 4.27 & 1.68 & 0.60 & 1.98 & 5.38 \\
\hline 4.5 & 4.25 & 1.63 & 0.67 & 2.17 & 5.21 \\
\hline 6.0 & 4.18 & 1.54 & 0.61 & 2.47 & 5.03 \\
\hline 7.5 & 4.11 & 1.52 & 0.57 & 2.72 & 4.82 \\
\hline 9.0 & 3.97 & 1.38 & 0.55 & 2.98 & 4.63 \\
\hline 10.5 & 4.06 & 1.62 & 0.63 & 3.22 & 4.41 \\
\hline 11.5 & 4.15 & 1.52 & 0.62 & 3.36 & 4.25 \\
\hline 13.0 & 3.81 & 1.50 & 0.58 & 3.56 & 4.07 \\
\hline 14.5 & 3.83 & 1.31 & 0.59 & 3.82 & 3.93 \\
\hline 16.0 & 3.79 & 1.21 & 0.57 & 4.07 & 3.76 \\
\hline 17.5 & 3.81 & 1.20 & 0.43 & 4.27 & 3.58 \\
\hline 19.0 & 3.77 & 1.09 & 0.41 & 4.45 & 3.42 \\
\hline 20.5 & 3.77 & 1.20 & 0.39 & 4.68 & 3.22 \\
\hline
\end{tabular}


Table B-2. Experiment 3 Constant Current Data for the Reduction of $\mathrm{NO}_{3}^{-}, \mathrm{NO}_{2}^{-}$, and $\mathrm{H}_{2} \mathrm{O}$ at a Nickel Cathode and the Oxidation of $\mathrm{OH}^{-}$at a Stainless Steel Anode.

\begin{tabular}{|c|c|c|c|c|c|}
\hline \multicolumn{4}{|c|}{ Electrode Materials: Nickel Cathode, Stainless Steel Anode } & \multicolumn{2}{|c|}{ Date:08/01/94-08/03/94 } \\
\hline \multicolumn{6}{|c|}{ Current Density: $0.25 \mathrm{~A} / \mathrm{cm}_{2}$} \\
\hline Electrolysis & Measured & Catholyte & Catholyte & Catholyte & Anolyte \\
\hline Time (Hours) & $\begin{array}{c}\text { Anode vs } \\
\text { Cathode }(\mathrm{V})\end{array}$ & $\begin{array}{c}\mathrm{NO}_{3}^{-} \text {Conc. } \\
\text { (M) }\end{array}$ & $\begin{array}{c}\mathrm{NO}_{2}^{-} \text {Conc. } \\
(\mathrm{M})\end{array}$ & $\begin{array}{l}\mathrm{OH}^{-} \text {Conc. } \\
\text { (M) }\end{array}$ & Conc. (M) \\
\hline 0 & 4.36 & 1.82 & 0.53 & 1.18 & 4.85 \\
\hline 2.0 & 4.28 & 1.71 & 0.51 & 1.54 & 4.65 \\
\hline 4.0 & 3.97 & 1.52 & 0.56 & 1.86 & 4.54 \\
\hline 6.0 & 3.67 & 1.44 & 0.51 & 2.23 & 4.23 \\
\hline 8.0 & 3.66 & 1.38 & 0.45 & 2.57 & 4.26 \\
\hline 10.0 & 3.83 & 1.28 & 0.45 & 2.84 & 3.91 \\
\hline 12.0 & 4.18 & 1.16 & 0.56 & 3.14 & 3.63 \\
\hline 14.0 & 3.53 & 1.14 & 0.51 & 3.33 & 3.33 \\
\hline 16.0 & 3.87 & 1.12 & 0.40 & 3.66 & 3.02 \\
\hline 18.0 & 3.85 & 1.06 & 0.45 & 3.87 & 2.00 \\
\hline 20.0 & 4.10 & 0.9995 & 0.4 & 4.09 & 2.32 \\
\hline
\end{tabular}

The experimental data given in Tables B-3 are for a full simulant batch run to $85 \%$ destruction of the $\mathrm{NO}_{3}-\mathrm{NO}_{2}$ - mixture composed initially of $2.043 \mathrm{MNaNO}_{3}, 0.634 \mathrm{MNaNO}$, and $1.47 \mathrm{M} \mathrm{NaOH}$. The run was conducted at a constant current density of $0.25 \mathrm{~A} / \mathrm{cm}^{2}$ until the off-gas composition reached $50 \% \mathrm{H}_{2}$ at which time (i.e., 20 hours) the current density was reduced to $0.05 \mathrm{~A} / \mathrm{cm}^{2}$ in order to produce more favorable conditions for the reduction of $\mathrm{NO}_{3}-1$ $\mathrm{NO}_{2}$-. The total duration of the experiment was 30 hours with initial volumes of the anolyte and catholyte of 4000 and $2000 \mathrm{ml}$. Additions of $150 \mathrm{ml}$ of $5 \mathrm{M} \mathrm{NaOH}$ were made to the anolyte at 2 hour intervals to assure an adequate supply of $\mathrm{NaOH}$ in the anolyte for complete destruction of the $\mathrm{NO}_{3}-\mathrm{NO}_{2}-$. The initial and operating conditions were taken from the numerical model by simulating the experimental runs. Catholyte off-gas samples were taken at 1 hour intervals for GC analysis and liquid samples were taken of the anolyte $(30 \mathrm{ml})$ and catholyte $(100 \mathrm{ml})$ at 2 hour intervals. The argon carrier gas flow rate was maintained at a constant $5 \mathrm{ml} / \mathrm{min}$ throughout the experiment. The catholyte liquid-phase samples were analyzed via ion chromatography for $\mathrm{NO}_{3}$ $\mathrm{NO}_{2}-$, by Kjeldahl analysis for the $\mathrm{NH}_{3}$, and by titration with oxalic acid for $\mathrm{OH}$. The anolyte 


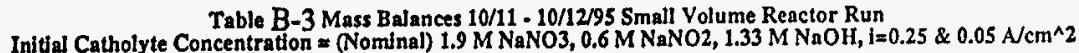

Nitrogen Atom Balance

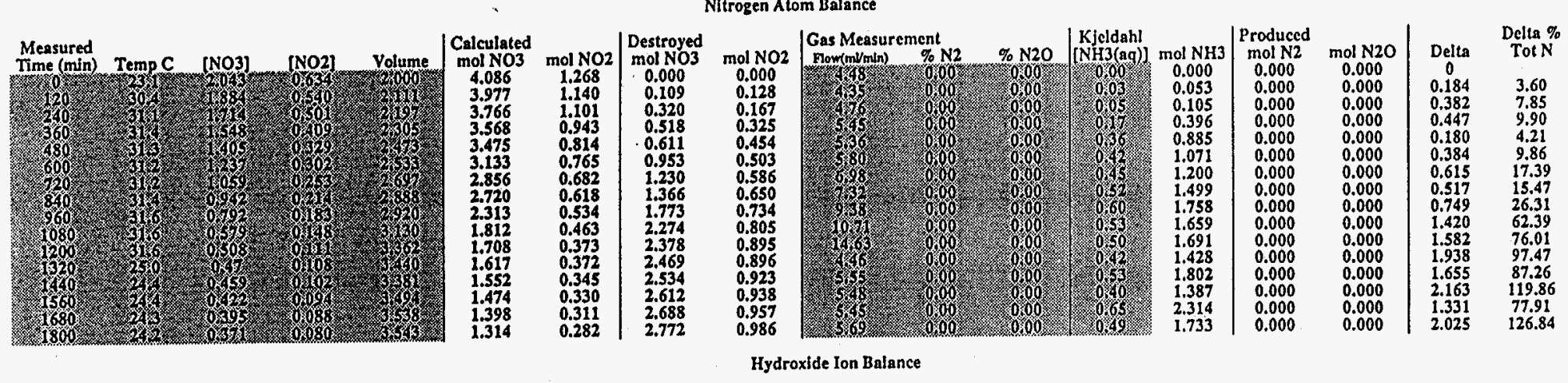

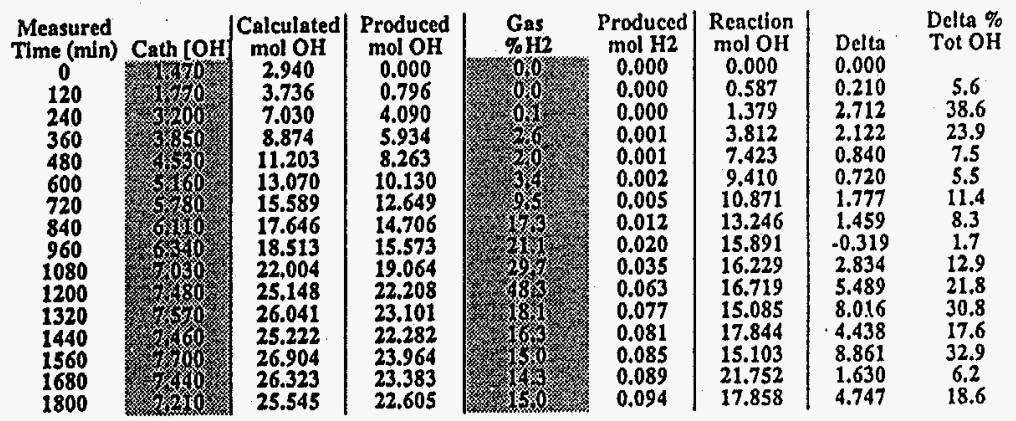

Potential \& Current Balance

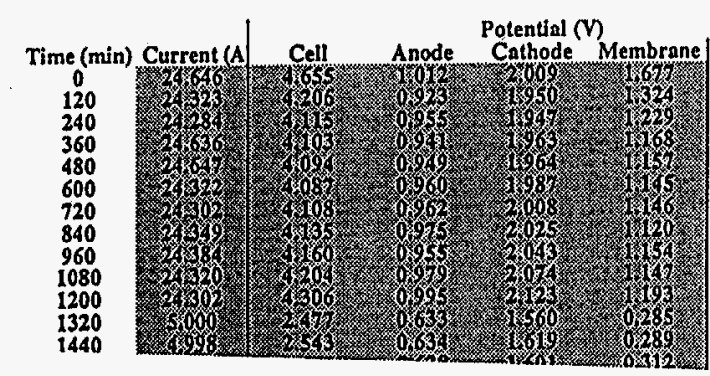

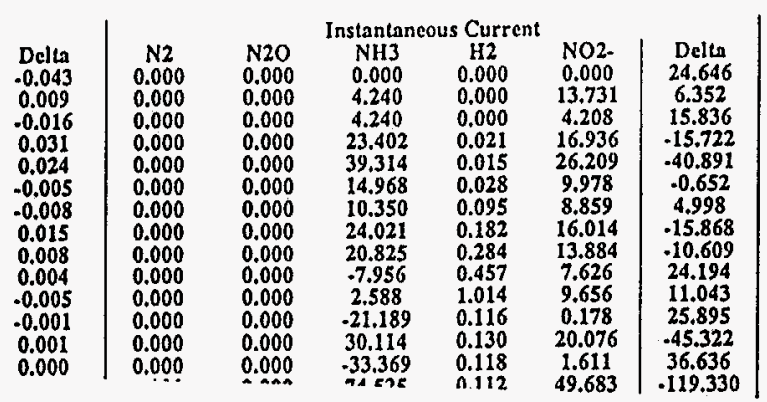


samples were titrated for $\mathrm{OH}^{-}$; and the initial and the final solutions were analyzed for $\mathrm{NO}_{3}-\mathrm{NO}_{2}-$ to determine the flux of these anions into the anolyte.

A mass balance for the molecular and the atomic species is shown in Table B-3.

Furthermore, a potential and current balance is shown at the bottom of the figure. The deviation of the analytically determined concentrations and molar off-gas flow rates from the calculated values indicates that the mass balance cannot by fully closed at the individual sampling intervals. Also, the mass balance cannot account for the flux of water or the ionic species through the Nafion $\otimes$ membrane. Fluxes will be determined from the model predictions. Model predictions showed that there would be no appreciable generations of $\mathrm{N}_{2}$ or $\mathrm{N}_{2} \mathrm{O}$, which was verified experimentally. The model also predicted a liquid-phase accumulation of $\mathrm{NH}_{3}$, which was observed experimentally.

\subsection{Appendix C \\ Mass Balance Calculations}

\section{Equations Used}

All of the techniques included to this point are used to generate the necessary numbers to complete the mass balance. Using the internal standard (Section $4 \& 5$ ) and the ion chromatography results the volume can be determined using equation 1 . The average of the two calculations is reported.

$$
V_{t}=\frac{V_{i} * \mathrm{ppmBr}_{i}^{-} \text {orCl }}{\mathrm{ppmBr}_{i}^{-} \text {or Cl} l_{i}^{-}}
$$

The obtained volume is then used to determine the total number of moles of nitrate, nitrite, and hydroxide present at every sampling time. The Kjeldahl determination gives the concentration of dissolved ammonia which is then likewise converted into total moles of ammonia. From the chromatographic determination of nitrate and nitrite, the moles of each destroyed as a function of time can be determined.

To obtain the moles of nitrogen gas and nitrous oxide, the ideal gas law is assumed to be in effect at room temperature which gives a molar volume of $22.4 \mathrm{~L}$ for all of the gaseous species. This is divided into the total volume of the gas evolved (estimated as a simple integral) over the sampling time interval using the instantaneous gas flows (see equation 2).

$$
\frac{\text { mole Gas }}{\text { Time Interval }}=\frac{\text { FlowRate }(\mathrm{ml} / \mathrm{min}) * \Delta \text { Time }(\mathrm{min})}{22400 \mathrm{ml}}
$$

With this information a nitrogen atom balance can be constructed. As part of this a Delta can be calculated as in equation C3. From this the fraction of unaccounted for nitrogen (Delta \% 
Total N) can be determined by dividing the absolute value of Delta by the initial nitrogen (Equation C4). Similarly, the analytical accuracy of this determination can be estimated by dividing the absolute value of Delta by the moles of nitrate and nitrite destroyed (Equation C5).

$$
\text { Delta }=\text { mol Dest } \mathrm{NO}_{2}^{-}, \mathrm{NO}_{3}^{-}-\left(\mathrm{molNH}_{3}+2 *\left(\mathrm{molN}_{2}+\operatorname{molN}_{2} \mathrm{O}\right)\right.
$$

A similar method is followed to construct a hydroxide balance. However, to determine the moles of hydroxide produced on must include the coefficients of the various nitrate and nitrite

$$
\begin{gathered}
\text { Delta } \% \text { Total } N=\frac{\text { Delta } 100}{m o l N_{\text {init }}} \\
\text { Relative Accuracy } \text { est }=\frac{\text { Delta }}{\text { mol Dest }_{\mathrm{NO}_{2}^{-}, \mathrm{NO}_{3}^{-}}}
\end{gathered}
$$

destruction reactions. This determination is closely tied on the nitrogen balance mentioned above. To determine the reaction produced hydroxide one uses equation C6 which incorporates all of the necessary coefficients as follows: For every nitrate destroyed-two hydroxides, for every ammonia-seven hydroxides, for every nitrogen molecule-eight hydroxides, for every nitrous oxide-six hydroxides, and for every hydrogen molecule-two hydroxides.

$$
\begin{aligned}
\text { molOH }^{-} & =2 * \operatorname{molNO}_{3}^{-} \text {Dest }+7 * \text { molNH }_{3} \\
& +8 * \text { molN }_{2}+6 * \mathrm{~N}_{2} \mathrm{O}+2 * \text { molH }_{2}
\end{aligned}
$$

\subsection{Acknowledgments}

This work was funded by the Department of Energy, Office of Technology Development, Office of Environmental Management through the Efficient Separations and Processing Integrated Program under contract DE-AC09-88SR18035; with Westinghouse Savannah River Company as task order number 112 through the SCUREF contract number AA009-00T. 